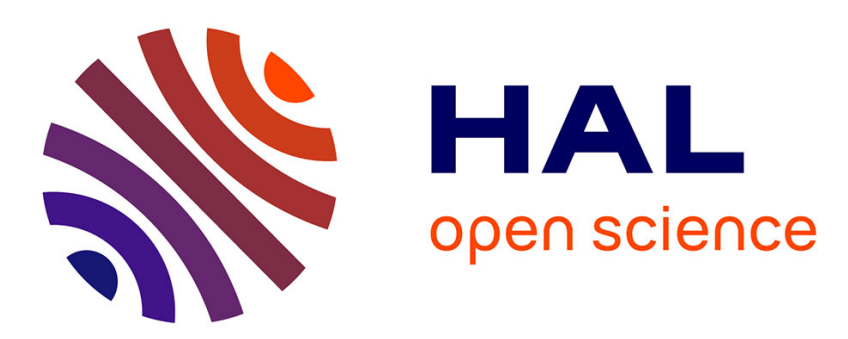

\title{
Closed-loop control of an open cavity flow using reduced-order models
}

\author{
Alexandre Barbagallo, D. Sipp, Peter J. Schmid
}

\section{To cite this version:}

Alexandre Barbagallo, D. Sipp, Peter J. Schmid. Closed-loop control of an open cavity flow using reduced-order models. Journal of Fluid Mechanics, 2009, 641 (December), pp.1-50. $10.1017 / \mathrm{s} 0022112009991418$. hal-01021129

HAL Id: hal-01021129

https://hal-polytechnique.archives-ouvertes.fr/hal-01021129

Submitted on 16 Jul 2014

HAL is a multi-disciplinary open access archive for the deposit and dissemination of scientific research documents, whether they are published or not. The documents may come from teaching and research institutions in France or abroad, or from public or private research centers.
L'archive ouverte pluridisciplinaire HAL, est destinée au dépôt et à la diffusion de documents scientifiques de niveau recherche, publiés ou non, émanant des établissements d'enseignement et de recherche français ou étrangers, des laboratoires publics ou privés. 


\title{
Closed-loop control of an open cavity flow using reduced-order models
}

\author{
ALEXANDRE BARBAGALLO ${ }^{1,2}$, \\ DENIS SIPP ${ }^{1}$ AND PETER J. SCHMID ${ }^{2} \dagger$ \\ ${ }^{1}$ ONERA-DAFE, 8 rue des Vertugadins, 92190 Meudon, France \\ ${ }^{2}$ Laboratoire d'Hydrodynamique (LadHyX), CNRS-Ecole Polytechnique, 91128 Palaiseau, France
}

(Received 30 October 2008; revised 23 July 2009; accepted 24 July 2009; first published online 30 November 2009)

The control of separated fluid flow by reduced-order models is studied using the twodimensional incompressible flow over an open square cavity at Reynolds numbers where instabilities are present. Actuation and measurement locations are taken on the upstream and downstream edge of the cavity. A bi-orthogonal projection is introduced to arrive at reduced-order models for the compensated problem. Global modes, proper orthogonal decomposition (POD) modes and balanced modes are used as expansion bases for the model reduction. The open-loop behaviour of the full and the reduced systems is analysed by comparing the respective transfer functions. This analysis shows that global modes are inadequate to sufficiently represent the input-output behaviour whereas POD and balanced modes are capable of properly approximating the exact transfer function. Balanced modes are far more efficient in this process, but POD modes show superior robustness. The performance of the closed-loop system corroborates this finding: while reduced-order models based on POD are able to render the compensated system stable, balanced modes accomplish the same with far fewer degrees of freedom.

Key words: flow control, instability

\section{Introduction}

Regions of separated fluid flow are a common feature of a great majority of realistic configurations. High-Reynolds-number flow about blunt bodies, airfoils during moderate and high angles of attack, curved engine inlets or engine nozzles or any occurrence of shock-boundary layer interactions are only a few examples where adverse pressure gradients or adverse geometries cause the flow to detach from the wall. Finite regions of separated flow commonly exhibit Kelvin-Helmholtz type instabilities that manifest themselves in an unsteady flow behaviour in the wake of the separation bubble. This behaviour, in turn, negatively affects the mean flow as well as the perturbation dynamics further downstream as it imprints a specific frequency on the subsequent flow. It is thus of great fundamental and technological interest to describe the dynamics of separated flows and design means to suppress its formation or - if separation is inevitable or prohibitively costly - to weaken its unsteadiness.

Because of its inherent complexity and marked sensitivity to specific flow and geometric conditions, results on the dynamics of separated flows are challenging to 
generalize. For this reason, one particular prototypical configuration that captures the dominant features of separated flows has received a great deal of attention and has acted as a proxy for a wide range of more complicated situations: the flow over an open cavity. Even though the majority of these studies has concentrated on the compressible flow over a cavity, in particular the generation of acoustic waves (e.g. Cattafesta et al. 2003; Rowley \& Williams 2006; Bres \& Colonius 2008; Cattafesta et al. 2008), we will focus on the incompressible flow over a square cavity. This type of flow exhibits a recirculating component (confined geometrically to the cavity) as well as a strong shear layer that forms at the top of the cavity and, for sufficiently high Reynolds number, becomes unstable and settles into a characteristic periodic motion (see Sipp \& Lebedev 2007). Experimental investigations on an equivalent three-dimensional configuration have been carried by Faure et al. (2007) emphasizing the three-dimensional development of the flow. An analysis of the dynamic behaviour for an open-cavity flow by traditional means is complicated by the complex geometry and, consequently, the lack of an easily available base flow profile. Rather, a global approach, both for the base flow and the perturbation dynamics, has to be employed in order to assess and quantify the stability characteristics of this type of flow.

The stability analysis of flows with more than one inhomogeneous coordinate direction has been first pursued by Zebib (1987) and Jackson (1987) who studied the stability characteristics of flow past a cylinder and other blunt bodies. These early studies were soon followed by investigations into the perturbation dynamics in cylinder wakes (e.g. Noack \& Eckelmann 1994; Zielinska \& Wesfreid 1995) using similar means. Since then, the concept of global stability analysis has made significant progress and gained in popularity due to the advent of iterative eigenvalue routines (such as the Arnoldi method; see Lehoucq \& Scott 1997; Lehoucq, Sorensen \& Yang 1998) that, coupled with flow solvers, provide efficient techniques to extract relevant stability information from simulations of the flow under consideration. This coupling of iterative methods with numerical simulations allows the quantitative description of the disturbance behaviour of any flow that can be simulated with a sufficient degree of fidelity. Among the many stability studies of separated flows we mention Ding \& Kawahara (1999) who studied flow in the wake of a cylinder, Barkley, Gomes \& Henderson (2002) who analysed flow over a backward-facing step and Ehrenstein \& Gallaire $(2005,2008)$ who treated the separated flow over a smooth bump as a global stability problem. Even the stability of more application-oriented geometries has recently been treated from a global perspective (e.g. Crouch, Garbaruk \& Magidov 2007 analysing the onset of transonic shock-buffeting; see Theofilis 2000 for a review of global stability analysis in aeronautical applications). In each case, the extracted global spectrum then represents the inherent dynamic behaviour of the fluid system (given by growth rates, phase velocities, transient growth potential, receptivity behaviour and their dependence on the governing parameters). Even the extraction of non-modal behaviour, such as optimal transient growth, has been accomplished by direct numerical simulations (DNS) feeding flow-field data into a direct-adjoint optimization algorithm (see Blackburn, Barkley \& Sherwin 2008 for an application to the flow over a backward-facing step, and Marquet et al. 2008 for flow in a curved channel). This type of analysis produces unprecedented stability information about complex flows and forms an important and imperative first step in the more challenging undertaking of manipulating the flow into a more desirable state or into a more acceptable dynamic behaviour.

Over the past years the control of fluid flow has come to prominence as a new and promising subject that combines our understanding of the dynamics of a fluid system with techniques to manipulate it (Bewley 2001; Kim 2003; Kim \& Bewley 2007). 
Remarkable progress has been made on generic configurations including full-state information control of turbulent channel flow (Moin \& Bewley 1994; Joshi, Speyer \& Kim 1997), partial-state information control of transitional channel flow (Högberg, Bewley \& Henningson 2003), estimation in wall-bounded shear flows (Hœpffner et al. 2005) and control of spatially growing boundary layers (Chevalier et al. 2007). The techniques applied in these situations have greatly relied on a mathematical framework established in control theory as described in standard references (e.g. Burl 1999; Zhou, Salomon \& Wu 2002), but additional complications had to be overcome when adapting them to fluid flows. Under even slightly more realistic circumstances, however, severe limitations due to the sheer size of the involved matrix equations have been encountered. It has thus been quickly realized that the application of approximate and iterative algorithms (as reviewed, for example, in Bai 2002 and Freund 2003), as well as the a priori reduction of the number of degrees of freedom (see e.g. Antoulas, Sorensen \& Gugercin 2001), are crucial techniques when attempting the control design for even moderately complex flows. Mathematically, model reduction can be described as a projection method based on a hierarchical (generally) bi-orthogonal expansion basis, and these techniques are now commonly applied to reduce a linear (or nonlinear) system. It is important to realize, however, that the choice of these fluid structures must be tailored to the application in mind; ignoring this fact can yield to suboptimal results at best, to ineffective control strategies at worst.

Global modes, i.e. the eigenvectors of the global stability problem, are often considered as the representation of the system's linear dynamics. The expression of this dynamics by a linear combination of global modes, hierarchically ordered by their decay rate, can yield a reduced model that accurately describes the inherent linear dynamics of the full system. As soon as forcing (representing either an external disturbance environment or control input) is applied or measurements are extracted from the system, the global mode expansion may no longer be appropriate for capturing the modified dynamics. This behaviour has been observed by Lauga \& Bewley (2003, 2004) where the fluid system has been replaced by the complex Ginzburg-Landau (cGL) equation and global modes have been used to reduce the system. The loss of stabilizability using global modes has then been linked to the domain of support of the adjoint global modes. An expansion of a larger-scale fluid system into global modes has been taken by Åkervik et al. (2007) for flow over a shallow cavity and by Ehrenstein \& Gallaire (2008) for flow over a smooth bump. A reduced-order controller has then been designed to stabilize the globally unstable flow (see also the review by Henningson \& Akervik 2008). In a similar effort, Ehrenstein \& Gallaire (2008) could stabilize a globally unstable flow using a reduced-order model based on global modes after they projected the control effort onto the basis of the reduced model. We will outline and investigate the steps involved in these control designs and, in particular, assess the effectiveness of this choice of basis in accomplishing a globally stable compensated system.

Proper orthogonal decomposition (POD) modes are popular in describing fluid dynamical systems by a reduced set of equations. This popularity stems from the simple manner of extracting them from numerical simulations, their orthogonality properties and their interpretation as energy-ranked coherent structures of the flow (see e.g. Lumley 1970; Sirovich 1987; Berkooz, Holmes \& Lumley 1993). POD is used in a variety of ways to analyse and describe complex fluid systems. Following their original objectives, POD modes describe a flow field in terms of coherent structures ranked by their inherent energy content. As such, POD is a pattern recognition tool that detects the most energetic fluid elements in a generally turbulent flow configuration. This type of flow analysis has been pursued by a great many researchers 
(see Berkooz et al. 1993; Podvin et al. 2006, among others) for numerically as well as experimentally generated data. It has quickly been recognized that the extracted POD modes are suitable for a low-order approximation of the flow behaviour. The argument that by using a POD-basis for the reduction of the full Navier-Stokes equations via a Galerkin expansion the vast majority of the system's energy can be captured has spawned a substantial body of literature on low-dimensional models for complex flow dynamics (see e.g. Noack et al. 2003; Buffoni, Camarri \& Iollo 2006; Galletti et al. 2007 and others). More recently, these low-dimensional models have been incorporated into flow control strategies (see Delville, Cordier \& Bonnet 1998; Tadmor et al. 2004; Bergman, Cordier \& Brancher 2006 for flow around a cylinder, or Samimy et al. 2007 for flow over an open cavity). It is commonly known that POD modes optimally express the driven dynamics of a fluid system; in mathematical terms, an expansion in POD modes that have been constructed from an impulse released from the control location produces optimal controllability of the reduced system. The application of POD modes to partial-state information control problems (where observability is equally important) may often lead to unsatisfactory results. Adjoint POD modes, on the other hand, produce optimal observability of the reduced model, but usually at the expense of controllability, which makes them less suited for control problems.

It has long been recognized that in order to reduce control problems to a desired size, both controllability, i.e. the ability of the applied forcing to reach flow states, and observability, i.e. the ability of flow states to register at the sensor locations, are equally important. An expansion basis that balances these two concepts would be particularly suited to express the flow of information from the actuator via the linear system to the sensor and thus yield a reduced model that could be subjected to optimal control design. These balanced modes have been introduced more than two decades ago (Moore 1981) and have been applied to small and moderately sized problems; even extensions to unstable systems (Zhou, Salomon \& Wu 1999) and nonlinear control problems (Scherpen 1993; Lall, Marsden \& Glavaski 2002) have been developed. The necessity to solve matrix Lyapunov and Sylvester equations, however, has precluded them from being applied to large-scale control problems. A recent development (see Rowley 2005 and earlier work by Willcox \& Peraire 2002), combining computational methodology from POD modes with a balancing procedure, has overcome this difficulty and has brought the model reduction of largescale control problems within reach of current computational technology. Since this breakthrough model reduction based on balanced POD modes has become a readily applied technique for the analysis of generic wall-bounded shear flows such as channel (Ilak \& Rowley 2006; Ahuja \& Rowley 2008; Ilak \& Rowley 2008) and boundary layer flow (Bagheri, Brandt \& Henningson 2009a) as well as of model equations (Bagheri et al. 2009b) mimicking fluid behaviour.

The article proceeds along the following outline. After introducing the flow configuration and details of the discretization and solution algorithm, a partitioning strategy into unstable and stable subspaces and a general bi-orthogonal projection method will be presented which allow the assessment of various commonly used expansion bases for the reduction of the full input-output behaviour to that of a system with significantly fewer degrees of freedom. Global modes will then be extracted from the flow via a shift-invert Arnoldi technique and used as a first expansion basis. Their effectiveness in representing the input-output behaviour, illustrated by the transfer function, will be critically evaluated and analysed. Expansion bases consisting of proper orthogonal modes as well as balanced modes will also be formed and evaluated using the same measure. By closing the control loop, the 
various expansion bases will then be examined as to their ability to control the unstable flow and yield a stable compensated system. A summary and discussion of the major findings, and details of derivations in form of an appendix, will conclude this article. The main contributions of this work comprise the application of the lifting procedure for flow in a complex geometry, the partitioning of the linearized flow behaviour into stable and unstable dynamics, a demonstration of the importance of modelling the dynamics in the stable subspace, the introduction of quantitative performance measures for the latter modelling effort and the performance assessment of closed-loop control schemes based on reduced-order models.

\section{Configuration and mathematical background}

\subsection{Configuration}

The two-dimensional configuration we consider in this article is sketched in figure 1(a). It consists of a uniform flow of velocity $U_{\infty}$ over a square cavity of depth $D$. The precise configuration with its geometric and numerical details is fully described in Sipp \& Lebedev (2007). The characteristic quantities of this configuration, i.e. $U_{\infty}, D$, as well as the dynamic viscosity $v$, are used as reference scales for non-dimensionalizing the governing two-dimensional incompressible Navier-Stokes equations. This results in the Reynolds number, defined as $R e=U_{\infty} D / \nu$. In the following, we choose $R e=7500$ so as to have an unstable configuration; the critical Reynolds number of $R e=4140$ has been determined in Sipp \& Lebedev (2007).

Before proceeding with the linear stability analysis and linear control design for this flow a base flow to linearize about has to be determined. This is accomplished by setting the unsteady terms of the two-dimensional Navier-Stokes equations to zero and solving the resulting nonlinear equations by a Newton-Raphson method. The base flow $\boldsymbol{u}_{0}=\left(u_{0}, v_{0}\right)$ displaying a shear layer above the cavity and a dominant cavity vortex is shown in figure $1(b)$, visualized by contours of the streamwise velocity and velocity vectors. Note that the boundary layer starts developing at $x=-0.4$ (the origin of our coordinate system coincides with the top left corner of the square cavity). The displacement thickness at the upstream edge of the cavity is $\delta_{1} \approx 0.012$, leading to a Reynolds number based on the displacement thickness of $R e_{\delta_{1}}=U_{\infty} \delta_{1} / v \approx 90$. This choice of parameters rules out instabilities related to the boundary layer dynamics.

\subsection{Governing equations in state-space form}

After a base flow given by the streamwise and normal velocities $\boldsymbol{u}_{0}=\left(u_{0}, v_{0}\right)$ has been determined, the linearized governing equations can be written in the form of an initial-value problem according to

$$
\mathscr{Q} \frac{\partial \mathscr{X}_{T}}{\partial t}=\mathscr{A} \mathscr{X}_{T}
$$

with

$$
\begin{gathered}
\mathscr{X}_{T}=\left(\begin{array}{c}
\boldsymbol{u}_{T} \\
p_{T}
\end{array}\right), \\
\mathscr{Q}=\left(\begin{array}{cc}
\mathscr{I} & 0 \\
0 & 0
\end{array}\right), \\
\mathscr{A}=\left(\begin{array}{cc}
-\nabla() \cdot \boldsymbol{u}_{0}-\nabla \boldsymbol{u}_{0} \cdot()+R e^{-1} \Delta & -\nabla \\
\nabla^{*} & 0
\end{array}\right)
\end{gathered}
$$


(a)

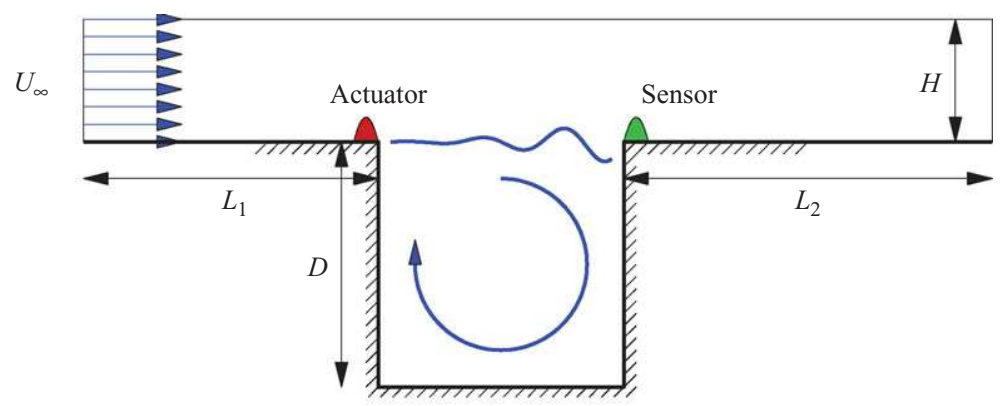

(b)

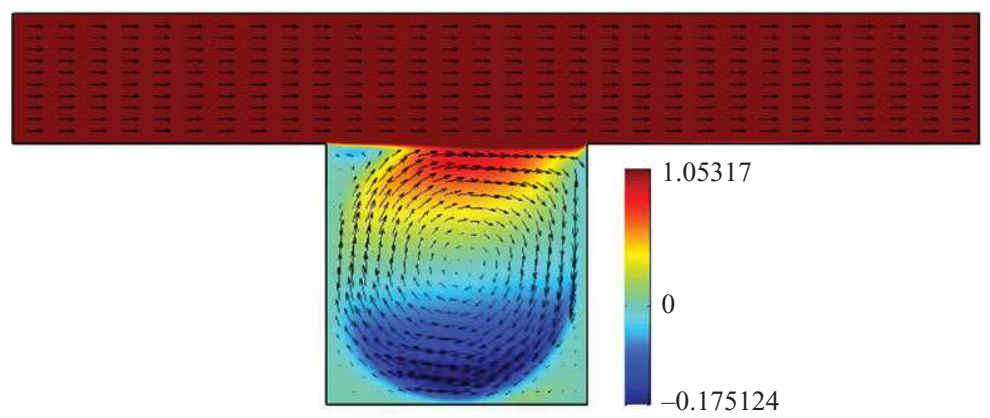

(c)

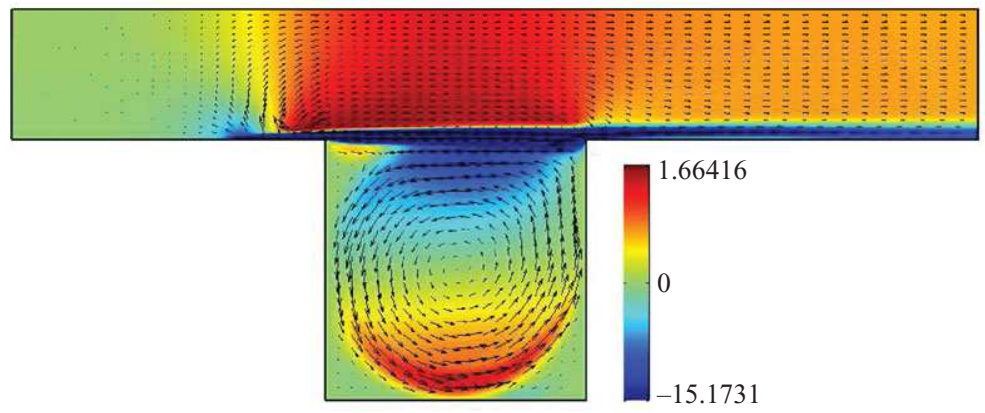

FIGURE 1. (a) Sketch of the geometry for flow over a square cavity. (b) Base flow, visualized by streamwise velocity contours and velocity vectors, for a Reynolds number of $R e=7500$. (c) Inhomogeneous solution $\mathbf{C}$ with a unit force at the actuator location, visualized by streamwise velocity contours and velocity vectors.

with $\boldsymbol{u}_{T}=\left(u_{T}, v_{T}\right)$ as the perturbation velocity vector, $p_{T}$ as the perturbation pressure and $\nabla, \nabla^{*}, \Delta=\nabla^{*} \nabla$ denoting the Cartesian gradient, divergence and Laplacian operator, respectively. The superscript ${ }^{*}$ stands for the complex conjugate transpose. The fluid enters the domain from the left of the domain (see figure 1a) where a uniform streamwise velocity $\left(u_{T}=1, v_{T}=0\right)$ is prescribed. On the top of the domain a symmetry boundary condition $\left(\partial_{y} u_{T}=0, v_{T}=0\right)$ is used. A no-slip $\left(u_{T}=0, v_{T}=0\right)$ condition is implemented starting at the location $(x=-0.4, y=0)$ while a symmetry condition (no stress) is used for $(-1.2<x<-0.4, y=0)$ and $(1.75<x<2.50, y=0)$; this will generate a boundary layer of moderate thickness at the upstream edge of the cavity. Finally, a standard outflow condition is prescribed on the right of the domain. More details can be found in Sipp \& Lebedev (2007). 
In view of treating a flow control problem, we decide on a blowing/suction strategy over a localized section $\partial \Omega_{C}$ of the wall. Mathematically, this translates into a modified boundary condition of the form $\boldsymbol{u}_{w}=\rho(t) \boldsymbol{u}_{G}$ where $\boldsymbol{u}_{G}$ describes the velocity profile of the injected or extracted flow, while $\rho(t)$ determines its magnitude and temporal behaviour. This boundary condition renders the control problem inhomogeneous, but a transformation, referred to as lifting, will allow us to formulate the control problem as a driven homogeneous problem which has both mathematical and numerical advantages. We take the state vector $\mathscr{X}_{T}(t)$ as the sum of two parts: a solution $\mathscr{X}$ of the homogeneous problem (i.e. with no control applied, $\rho(t)=0$ ) and a solution $\mathscr{C}$ of the steady but inhomogeneous problem (i.e. with the specific constant control $\rho(t)=1$ applied). We then express the state vector in the form $\mathscr{X}_{T}(t)=\rho(t) \mathscr{C}+\mathscr{X}(t)$ and, upon substitution into the governing equation (2.1), obtain

$$
\mathscr{2} \frac{\partial \mathscr{X}}{\partial t}=\mathscr{A} \mathscr{X}+\mathscr{Q} \mathscr{C} c
$$

where $c=-\mathrm{d} \rho / \mathrm{d} t$.

For the actuator we assume a localized region near the upstream edge of the cavity (see figure $1 a$ ) and impose a parabolic normal velocity field over its streamwise extent $(-7 / 20 \leqslant x \leqslant 0, y=0)$. The profile is given as

$$
\begin{gathered}
u_{T}(x, y=0, t)=0 \\
v_{T}(x, y=0, t)=-\frac{x(1600 x+560)}{147} \rho(t)
\end{gathered}
$$

with the scalings chosen such that $\left(u_{T}=0, v_{T}=\rho\right)$ at the centre of the actuator's support. The associated control function $\mathscr{C}$ stemming from the above-mentioned lifting procedure is shown in figure $1(c)$, visualized by contours of the streamwise velocity as well as velocity vectors.

What remains in the formulation of the complete control problem is the specification of a measured quantity which yields information about the flow for the design of an estimator. As the measured quantity we take the wall-normal shear stress evaluated at and integrated over a localized region $\partial \Omega_{M}$ of the wall (the sensor location). We obtain

$$
m=\mathscr{M} \mathscr{X}=\left.\int_{\partial \Omega_{M}} \boldsymbol{t} \cdot \nabla \boldsymbol{u} \cdot \boldsymbol{n}\right|_{w} \mathrm{~d} s=\left.\int_{x=1}^{x=1.1} \frac{\partial u}{\partial y}\right|_{y=0} \mathrm{~d} x,
$$

where $\boldsymbol{n}$ is the inward unit normal on $\partial \Omega_{M}$ and $\boldsymbol{t}$ denotes the associated unit tangential vector. The sensor is located near the downstream edge of the cavity, on the segment $(1 \leqslant x \leqslant 1.1, y=0)$. Note that $m(t)=m_{T}(t)-\rho(t) m_{C}$ where $m_{T}(t)$ and $m_{C}$ are the measures related to $\mathscr{X}_{T}(t)$ and $\mathscr{C}$.

Finally, an energy based inner product of the form

$$
\langle\mathscr{Y}, \mathscr{X}\rangle \equiv \iint_{\Omega} \mathscr{Y} \cdot \mathscr{Q} \mathscr{X} \mathrm{d} \Omega
$$

will be used for projections.

This concludes the formulation of the continuous flow control problem in statespace form. We then proceed to discretize the problem. To this end, we mesh the domain by an unstructured finite-element grid to properly resolve near-wall and highshear features and employ $\left(P_{2}, P_{2}, P_{1}\right)$ finite elements, in conjunction with a weak formulation of the above equations, to arrive at a discretized form of the governing 
equations (2.3). We obtain

$$
\begin{aligned}
\boldsymbol{Q} \frac{\mathrm{d} \boldsymbol{X}}{\mathrm{d} t} & =\boldsymbol{A} \boldsymbol{X}+\boldsymbol{Q} \boldsymbol{C} c, \\
m & =\boldsymbol{M} \boldsymbol{X}, \\
\boldsymbol{X}_{T}(t) & =\rho(t) \boldsymbol{C}+\boldsymbol{X}(t), \\
\langle\boldsymbol{Y}, \boldsymbol{X}\rangle & =\boldsymbol{Y}^{*} \boldsymbol{Q} \boldsymbol{X}
\end{aligned}
$$

with

$$
\begin{gathered}
\boldsymbol{A C}=0, \\
\boldsymbol{C}=\boldsymbol{C}_{G} \quad \text { on } \partial \Omega_{C} .
\end{gathered}
$$

Again, the superscript ${ }^{*}$ represents the complex conjugate transpose. Assuming $n$ degrees of freedom for the state vector $\boldsymbol{X}$ which includes the two velocity components and the pressure, the above matrices are of the following size: $\boldsymbol{Q}, \boldsymbol{A} \in \mathbb{R}^{n \times n}, \boldsymbol{C} \in \mathbb{R}^{n \times 1}$, $\boldsymbol{M} \in \mathbb{R}^{1 \times n}$ and $c, m \in \mathbb{R}$. A typical discretization yields about 900000 degrees of freedom stemming from about 200000 triangles.

\section{Perturbation dynamics and global mode analysis}

The discretized system of equations (2.8) allows the computation of the temporal global spectrum and the associated global modes via the common assumption of an exponential time-dependence, i.e. $\boldsymbol{X}(x, y, t)=\tilde{\boldsymbol{X}}(x, y) \mathrm{e}^{\lambda t}$. We consequently obtain a generalized eigenvalue problem for $\lambda \in \mathbb{C}$ and $\tilde{X}$ of the form

$$
\lambda \boldsymbol{Q} \tilde{\boldsymbol{X}}=\boldsymbol{A} \tilde{\boldsymbol{X}},
$$

which has to be solved by iterative numerical techniques.

\subsection{Numerical method}

The solution of this eigenvalue problem by the direct QZ-algorithm is rather costly due to the size of the matrices $\boldsymbol{Q}$ and $\boldsymbol{A}$. Instead, we apply an iterative Krylov subspace technique, more precisely, the shift-invert Arnoldi algorithm, to extract information about the global spectrum and the corresponding global modes. The inversion is performed using a multifrontal sparse LU solver (MUMPS, see Amestoy et al. 2001).

Computations of global modes for flows in complex geometries are generally challenging and care must be exercised to avoid spurious or insufficiently converged eigenvalues and to still extract all relevant global modes. In our case, for each specified shift location 100 eigenvalues have been computed in its neighbourhood, after which the shift is moved to sweep over a sufficiently large part of the complex plane. For each choice of shift location both the direct and the adjoint eigenvalue problem has been solved, and particular care has been exercised to take into account each computed mode only once.

\subsection{Direct global modes}

The eigenvalues $\lambda=\sigma+\mathrm{i} \omega$ of the open cavity flow at $R e=7500$ are displayed in figure 2(a). The horizontal and vertical axes correspond, respectively, to the amplification rate $\sigma$ and the frequency $\omega$. The figure is symmetric with respect to $\omega=0$, and eigenvalues in the half-plane $\sigma>0$ represent unstable eigenmodes. In our computations the plane $\omega \geqslant 0$ contains 2630 eigenvalues with a total of 5166 eigenvalues in the entire plane. 
(a)

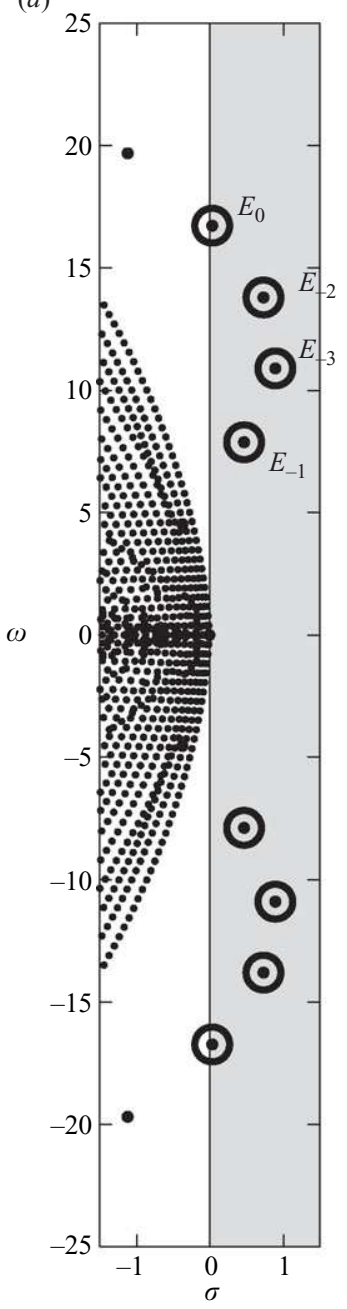

(b)

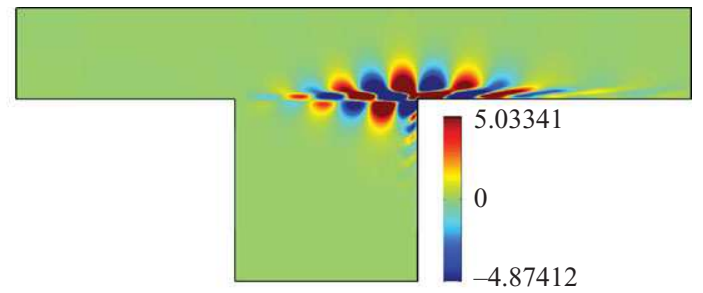

(c)

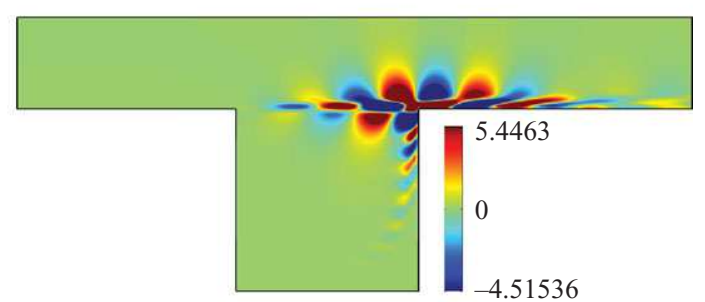

(d)

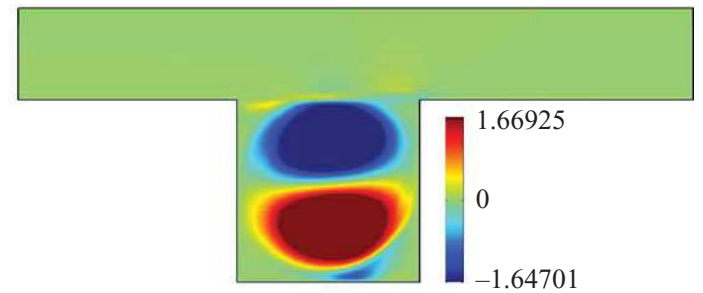

(e)

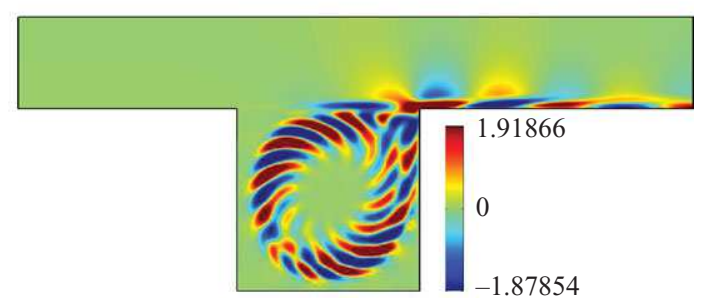

FIGURE 2. (a) Part of the global spectrum for flow over a square cavity at $R e=7500$, showing four unstable global modes (marked by circles and labelled $E_{-3}, E_{-2}, E_{-1}, E_{0}$ ) and their symmetric counterparts. $(b-e)$ Direct global modes corresponding to $E_{-3}(b), E_{-1}(c), E_{1}(d)$ and $E_{2}(e)$. The global modes are visualized by their streamwise velocity component.

We observe that there are four unstable global modes in the half-plane $\omega \geqslant 0$, which yields a total of eight unstable global modes in the complete plane. The four unstable global modes in the upper half-plane are denoted by $E_{-3}, E_{-2}, E_{-1}, E_{0}$. The amplification rate and frequency of these modes are listed in table 1, and the global modes corresponding to the eigenvalues $E_{-3}$ and $E_{-1}$ are displayed in figure $2(b, c)$. In each subplot we have represented the global structure by the horizontal $u$-velocity component. In figure $2(b)$ we observe that the most unstable global mode is composed of a series of counter-rotating vortices located atop the shear layer separating the outer and inner cavity flow. The eigenvector increases in amplitude as one moves downstream along the shear-layer and is most pronounced at the downstream edge of the cavity. Figure 2(c) represents similar results for the modal structure associated 


$\begin{array}{lcccccr}\text { Number } & \sigma & \omega & \hat{\boldsymbol{M}}_{i} & \operatorname{Re}\left(\hat{\boldsymbol{C}}_{i}\right) & \operatorname{Im}\left(\hat{\boldsymbol{C}}_{i}\right) & \Gamma_{i} \\ E_{-3} & 0.890 & 10.9 & 99.1 & -1.65 & 0.764 & \\ E_{-2} & 0.729 & 13.8 & 84.2 & -2.32 & 0.160 & \\ E_{-1} & 0.466 & 7.88 & 113.7 & -1.14 & 1.97 & \\ E_{0} & 0.0324 & 16.73 & 73.1 & -2.84 & -0.155 & \\ E_{1} & -0.00811 & 0 & 1.32 & 0.605 & 0 & 98 \\ E_{2} & -0.373 & 4.54 & 37.6 & -6.48 & 9.32 & 1142 \\ E_{3} & -0.716 & 0.203 & 10.7 & -3.81 & 9.21 & 148 \\ E_{4} & -1.12 & 19.7 & 64.1 & -2.99 & -0.499 & 173 \\ E_{5} & -2.70 & 22.7 & 50.7 & -3.27 & -1.84 & 71 \\ E_{6} & -5.01 & 2.71 & 0.0100 & 0.740 \cdot 10^{5} & 3.71 \cdot 10^{5} & 758 \\ E_{7} & -6.22 & 17.4 & 0.0104 & -3.13 \cdot 10^{2} & 2.54 \cdot 10^{2} & 0.67 \\ E_{8} & -9.65 & 12.4 & 6.47 \cdot 10^{-6} & 0.592 \cdot 10^{9} & -8.15 \cdot 10^{9} & 5477\end{array}$

TABLE 1. List of selected global eigenvalues together with variables quantifying their contribution in representing the flow's input-output behaviour (see text for an explanation).

with $E_{-1}$. We observe that the number of counter-rotating vortices is lower than for $E_{-3}$ which agrees with the fact that $E_{-1}$ has a lower eigenfrequency than $E_{-3}$.

Owing to the abundance of stable global modes, we will focus on two specific eigenvalues numbered $E_{1}$ and $E_{2}$ that are listed in table 1 together with various characteristic data. The least stable eigenmode among the damped eigenmodes (denoted in the spectrum by $E_{1}$ ) is depicted in figure $2(d)$ and consists of a weakly damped large-scale vortical structure located inside the cavity. Figure $2(e)$ focuses on the global mode corresponding to $E_{2}$ which is damped and oscillatory. It is characterized by features both inside the cavity and outside. Inside the cavity, it displays small-scale perturbations convected by the rigidly rotating base-flow. Outside, this structure connects to vortical elements in the base shear-layer that propagate downstream past the cavity.

In anticipation of further computations, the global modes will be gathered into a matrix $\boldsymbol{E}$, which is the solution of the generalized eigenproblem

$$
A E=Q E \Lambda,
$$

where $\boldsymbol{\Lambda}$ is a diagonal matrix of dimension $n \times n$ containing the $n$ eigenvalues. Hence, each column $j$ of $\boldsymbol{E}$ is an eigenvector corresponding to $\boldsymbol{\Lambda}_{j j}$. Since $\boldsymbol{A}$ is a real matrix, the eigenvalues are real or appear as complex conjugate pairs. The ordering and grouping of the eigenvalues and eigenvectors in $\boldsymbol{\Lambda}$ and $\boldsymbol{E}$ respect this fact, e.g. $\boldsymbol{\Lambda}_{11}=0.890+10.9 \mathrm{i}$, $\boldsymbol{\Lambda}_{22}=0.890-10.9 \mathrm{i}, \boldsymbol{\Lambda}_{99}=-0.00811$, etc. Furthermore, the eigenvectors are normalized such that their energy $\boldsymbol{E}_{j}^{*} \boldsymbol{Q} \boldsymbol{E}_{j}$ is one and their phase $\boldsymbol{M} \boldsymbol{E}_{j}$ is real and positive.

\subsection{Physical explanation of disturbance dynamics}

A DNS of the linearized Navier-Stokes equations (2.8a), based on a second-order accurate time marching scheme, has been used to gain further insight into the physics of these unstable global modes. The results of the simulation using the most unstable global mode $E_{-3}$ as an initial condition along with $c(t)=0$ are displayed in figure 3 where we show a spatio-temporal diagram of the pressure $p(x, y=0, t)$ in the $(x, t)$ plane.

Starting with an unstable initial condition the pressure increases exponentially. On the shear layer we observe vortical structures associated with low and high 


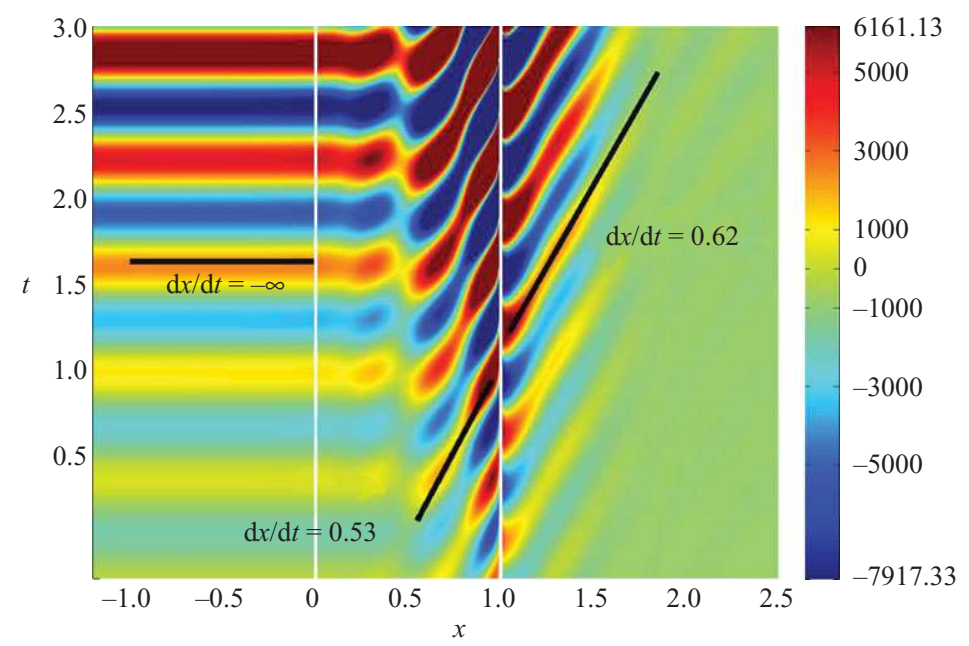

FIGURE 3. Spatio-temporal $x$-t diagram of pressure $p(x, y=0, t)$ contours with no control applied. The initial condition corresponds to the most unstable global mode. The two vertical white lines indicate the cavity walls. See also movie I in the online version of the paper.

pressure zones that are convected at the speed $\mathrm{d} x / \mathrm{d} t=0.53$, i.e. approximately half the free-stream base-flow speed. Once the vortical structures have left the cavity zone, they accelerate and reach a convective speed of $\mathrm{d} x / \mathrm{d} t=0.62$, which falls within the range of the free-stream and the low speeds that are present in the boundary layer. The cavity flow is globally unstable based on a classical feedback loop: the vortical structures are convected downstream in the shear layer at a speed of 0.53 ; during this phase they grow due to a Kelvin-Helmholtz instability (an inflection point is present in the base-flow velocity profile $u_{0}(x=$ const, $y)$ near $\left.y=0\right)$. These vortical structures subsequently impact the downstream cavity edge and generate an infinitely fast pressure wave $(\mathrm{d} x / \mathrm{d} t=-\infty)$ which travels upstream, excites the shear layer near the upstream edge of the cavity and regenerates perturbations that grow again due to a Kelvin-Helmholtz instability (see also Åkervik et al. 2007).

The general objective is then to stabilize the flow using a closed-loop control. Mathematically this corresponds to moving the unstable eigenvalue of $\boldsymbol{A}$ into the stable half-plane. We first, however, have to introduce reduced-order models on which our control design will depend.

\section{Model reduction}

Flows in complex geometries are characterized by a large number of degrees of freedom and standard (direct) techniques for computing stability characteristics or control schemes can no longer be applied. Instead, a projection onto relevant fluid structures, known as model reduction, has to be employed to reduce the size of the full problem to a more manageable number of degrees of freedom. For this reason, the model reduction techniques and algorithms play an increasingly central role as we attempt to control progressively complex flows. The choice of relevant fluid structures onto which our full system is projected is not obvious; a significant part of this paper is devoted to this important question. 


\subsection{Bi-orthogonal projection}

We will develop a mathematical framework that allows the model reduction of any system in state-space form using a general bi-orthogonal projection basis. This framework then allows the evaluation and analysis of various reduction techniques, e.g. based on global modes, optimally controllable (POD) modes or balanced proper orthogonal decomposition (BPOD) modes.

We start by introducing a general basis given by $\boldsymbol{V}$ and its dual equivalent $\boldsymbol{W}$ that satisfy the bi-orthogonality relation based on $\boldsymbol{Q}$ of the form

$$
W^{*} Q \boldsymbol{V}=I \text {, }
$$

where $\boldsymbol{V}, \boldsymbol{W} \in \mathbb{C}^{n \times q}$ and $\boldsymbol{I}$ stands for the $q \times q$ identity matrix. The parameter $q$ denotes the dimensionality of the projection basis. The discretized state vector $\boldsymbol{X}$ is then expressed as a linear combination of the columns of the chosen expansion basis $\boldsymbol{V}$ according to

$$
\begin{aligned}
\boldsymbol{X} & =\boldsymbol{V} \hat{\boldsymbol{X}}, \\
\hat{\boldsymbol{X}} & =\boldsymbol{W}^{*} \boldsymbol{Q} \boldsymbol{X},
\end{aligned}
$$

and the coefficients of this linear combination are contained in the vector $\hat{\boldsymbol{X}} \in \mathbb{C}^{q \times 1}$ which can be determined by invoking the bi-orthogonality condition. A straightforward Petrov-Galerkin projection then yields the reduced system

$$
\begin{aligned}
\frac{\mathrm{d} \hat{\boldsymbol{X}}}{\mathrm{d} t} & =\hat{\boldsymbol{A}} \hat{\boldsymbol{X}}+\hat{\boldsymbol{C}} c, \\
m & =\hat{\boldsymbol{M}} \hat{\boldsymbol{X}}
\end{aligned}
$$

with the reduced system, control and measurement matrices given as

$$
\begin{aligned}
& \hat{A}=W^{*} A V, \\
& \hat{C}=W^{*} Q C, \\
& \hat{M}=M V .
\end{aligned}
$$

Depending on the bases used for the model reduction, the above system should be able to represent critical features of the full system and capture the dynamics relevant to a successful control design.

\subsection{Basis consisting of global modes}

The basis $\boldsymbol{E}$ consisting of $n$ eigenvectors is non-orthogonal with respect to the scalar product based on $\boldsymbol{Q}$. This fact is a consequence of the non-normality of the system matrix $\boldsymbol{A}$, and the source of this non-normality lies in the convective terms of the linearized Navier-Stokes operator. Consequently, a second set of vectors - the dual basis $\boldsymbol{F}$ in the general framework - has to be determined to complete the bi-orthogonal projection. This basis may be obtained by solving for the eigenvalues and eigenvectors of the Hermitian of the linearized Navier-Stokes matrix, i.e.

$$
\boldsymbol{A}^{*} \boldsymbol{F}=\boldsymbol{Q} \boldsymbol{F} \boldsymbol{\Lambda}^{*} .
$$

The above eigenvalue problem produces eigenvalues that are complex conjugate to the eigenvalues of the previous problem. Hence, under the same ordering and grouping each column $j$ of the $n \times n$ matrix $\boldsymbol{F}$ is associated with the $j$ th column of $\boldsymbol{E}$ and the eigenvalue $\boldsymbol{\Lambda}_{j j}$. It can easily be verified that the two bases $\boldsymbol{E}$ and $\boldsymbol{F}$ are bi-orthogonal and that $\boldsymbol{F}$ may be normalized such that

$$
F^{*} Q E=I .
$$


Although the adjoint system is characterized by a spectrum that is complex conjugate to the direct spectrum, the corresponding adjoint eigenvectors differ significantly from their direct counterparts, owing to the non-normal nature of the underlying linearized Navier-Stokes equations. The adjoint global modes (not shown) are similar to their direct counterparts when considering reversing the base flow direction; the amplitude increases in magnitude as one moves upstream along the shear-layer and is largest at the upstream edge of the cavity. These observations confirm the non-normality of the linearized Navier-Stokes operator as the direct global mode is convected downstream whereas its adjoint equivalent propagates upstream.

\subsection{Stable subspace based on BPOD or POD basis}

The present paper treats a globally unstable flow. As we have seen, eight unstable global modes exist for our parameter settings. A partition of the full dynamics into two subspaces, one containing the unstable dynamics (based on the unstable global modes) and another describing the stable dynamics is required for the subsequent analysis. Since the dynamics within the unstable and stable subspaces are decoupled, they can be modelled separately.

Because of their ability to model the inherent instability (see $\S 3$ ) and because of their low dimensionality, the unstable global modes can be used directly to represent the dynamics in the unstable subspace (see Ahuja \& Rowley 2008). This procedure leads to an 'exact' model for this subspace in the sense that no modelling assumptions have to be invoked. For the stable subspace, on the other hand, a variety of choices are available.

We proceed by partitioning the eigenvectors $\boldsymbol{E}$ according to $\boldsymbol{E}=\left(\boldsymbol{E}_{u} \boldsymbol{E}_{s}\right)$ where $\boldsymbol{E}_{u}$ (resp. $\boldsymbol{E}_{s}$ ) of dimension $n \times 8$ (resp. $n \times(n-8)$ ) contains the eight unstable modes (resp. remaining stable modes). Similarly, $\boldsymbol{F}$ is decomposed as $\boldsymbol{F}=\left(\boldsymbol{F}_{u} \boldsymbol{F}_{s}\right)$ and satisfies the bi-orthogonality relation $\boldsymbol{F}_{u}^{*} \boldsymbol{Q} \boldsymbol{E}_{u}=\boldsymbol{I}$. Finally, the eigenvalue matrix $\boldsymbol{\Lambda}$ is split according to

$$
\boldsymbol{\Lambda}=\left(\begin{array}{cc}
\boldsymbol{\Lambda}_{u} & 0 \\
0 & \boldsymbol{\Lambda}_{s}
\end{array}\right),
$$

where the dimension of $\boldsymbol{\Lambda}_{u}$ and $\boldsymbol{\Lambda}_{s}$ is $8 \times 8$ and $(n-8) \times(n-8)$, respectively. In what follows, the subscripts ${ }_{u}$ will refer to quantities of the unstable subspace while the subscript ${ }_{s}$ will refer to quantities that are defined in the stable subspace.

We proceed by forming the projection matrices $\boldsymbol{P}_{u}$ onto the unstable and $\boldsymbol{P}_{s}$ onto the stable subspace as $\boldsymbol{P}_{u}=\boldsymbol{E}_{u} \boldsymbol{F}_{u}^{*} \mathbf{Q}$ and $\boldsymbol{P}_{s}=\boldsymbol{I}-\boldsymbol{E}_{u} \boldsymbol{F}_{u}^{*} \boldsymbol{Q}$. In the case the unstable subspace is modelled by the unstable global modes, a general projection basis can be written as $\boldsymbol{V}=\left(\boldsymbol{E}_{u} \boldsymbol{V}_{s}\right)$ and $\boldsymbol{W}=\left(\boldsymbol{F}_{u} \boldsymbol{W}_{s}\right)$ where $\boldsymbol{V}_{s}$ is defined as a basis belonging to the null-space of $\boldsymbol{P}_{u}$, and the relations $\boldsymbol{W}_{s}^{*} \boldsymbol{Q} \boldsymbol{E}_{u}=0$ and $\boldsymbol{W}_{s}^{*} \mathbf{Q} \boldsymbol{V}_{s}=\boldsymbol{I}$ can be easily verified.

The matrices (defined in (4.4)) appearing in the reduced-order model can now be rewritten as

$$
\hat{\boldsymbol{A}}=\left(\begin{array}{cc}
\boldsymbol{\Lambda}_{u} & 0 \\
0 & \hat{\boldsymbol{A}}_{s}
\end{array}\right), \quad \hat{\boldsymbol{C}}=\left(\begin{array}{c}
\hat{\boldsymbol{C}}_{u} \\
\hat{\boldsymbol{C}}_{s}
\end{array}\right), \quad \hat{\boldsymbol{M}}=\left(\hat{\boldsymbol{M}}_{u} \hat{\boldsymbol{M}}_{s}\right)
$$

where $\hat{\boldsymbol{A}}_{s}=\boldsymbol{W}_{s}^{*} \boldsymbol{A} \boldsymbol{V}_{s}$ is, in general, a dense matrix, and the control and measurement submatrices are respectively given as $\hat{\boldsymbol{C}}_{u}=\boldsymbol{F}_{u}^{*} \boldsymbol{Q C}, \hat{\boldsymbol{C}}_{s}=\boldsymbol{W}_{s}^{*} \mathbf{Q C}, \hat{\boldsymbol{M}}_{u}=\boldsymbol{M} \boldsymbol{E}_{u}$ and $\hat{\boldsymbol{M}}_{s}=\boldsymbol{M} \boldsymbol{V}_{s}$.

The stable subspace is usually high dimensional, and the main effort in reducing the system's dimension is expended reducing the dynamics in the stable subspace. 
Anticipating the results of $\S 6$, we assert that the modelling of the stable subspace is essential when it comes to effective closed-loop control design. The stable subspace contains substantial physical information about the overall dynamics, and we will show that extracting the relevant dynamical features from this subspace is pivotal for the success of any control strategy.

In control theory, it has long been recognized (see Zhou et al. 2002 and Antoulas 2005) that the transfer of information from the actuator to the sensor - the inputoutput behaviour - is the critical quantity that has to be carefully taken into consideration. Recent studies of Bagheri et al. (2009b) and Bagheri et al. (2009a) support this statement within a fluid mechanics framework. This statement then provides the motivation for the following section where the ability of various bases to reproduce the input-output behaviour of the stable subspace will be critically assessed and quantified. Various bases consisting of stable global modes, POD modes and BPOD modes will be successively considered and evaluated. At this point, we would like to point out that a reduced model $\hat{\boldsymbol{A}_{S}}$ of the stable subspace dynamics based on a Petrov-Galerkin projection does not necessarily have to result in a stable reduced system. Since the stability of the reduced-order models is critical to the success of closed-loop control, particular attention will be given to this property and its preservation through the model reduction process.

\section{Open-loop behaviour}

\subsection{Input-output behaviour}

In closed-loop mode the actuator manipulates the flow in order to reduce the perturbation growth and suppress the instability. Its action is, however, not limited to the unstable perturbations, but also triggers some stable states which are subsequently detected by the sensor. As we will see in the next section, this triggering process can constitute a source of failure for the controller. It is therefore important to understand and model these stable structures which are both excited by the actuator and observed by the sensor; in other words, we have to concern ourselves with the input-output behaviour of the stable subspace.

In this section our goal is to quantify the accuracy of various reduced models in capturing the input-output behaviour of the stable subspace. During this study we will thus consider the equations governing only the stable dynamics, i.e.

$$
\begin{aligned}
\boldsymbol{Q} \frac{\mathrm{d} \boldsymbol{X}}{\mathrm{d} t} & =\boldsymbol{A}_{s} \boldsymbol{X}+\boldsymbol{Q} \boldsymbol{C}_{s} c, \\
m & =\boldsymbol{M} \boldsymbol{X},
\end{aligned}
$$

where $\boldsymbol{A}_{s}=\boldsymbol{A P}_{s}$ and $\boldsymbol{C}_{s}=\boldsymbol{P}_{s} \boldsymbol{C}$. The above set of equations represents the system governed by (2.8) but projected onto the stable subspace.

By definition, the input-output behaviour links the effect of the actuator on the flow to the information extracted by the sensor. It can be described by the impulse response or, equivalently, by the transfer function. The impulse response, i.e. the reaction of the system to a Dirac function for the control law $c(t)=\delta(t)$, produces the measurement $m(t)=\boldsymbol{M} \boldsymbol{X}(t)$ where $\boldsymbol{X}$ is the solution of the initial-value problem

$$
\begin{aligned}
\boldsymbol{Q} \frac{\mathrm{d} \boldsymbol{X}}{\mathrm{d} t} & =\boldsymbol{A}_{s} \boldsymbol{X}, \\
\boldsymbol{X}(t=0) & =\boldsymbol{C}_{s} .
\end{aligned}
$$



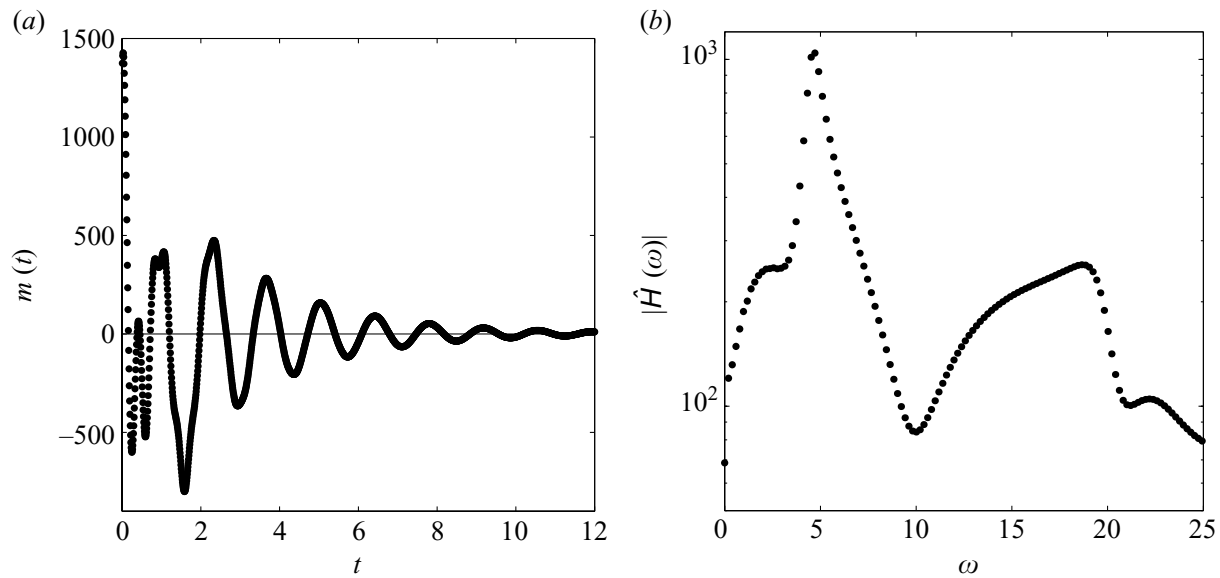

FIGURE 4. Impulse response $(a)$ as a function of time and transfer function $(b)$ as a function of frequency for the stable part of the flow over an open cavity at $R e=7500$.

In figure 4(a), this impulse response is displayed. In the corresponding simulation, 2.8(a) is integrated in time using a second-order accurate scheme, the initial condition is the control matrix $\boldsymbol{C}$ and the control law $c(t)=0$. After each time step the solution is projected, using the matrix $\boldsymbol{P}_{s}$, onto the stable subspace before the computation is advanced in time. Strictly speaking, only the initial condition would have to be projected; however, to avoid the amplification of numerical errors due to components in the unstable subspace, the above projection technique is employed at every time step. A first crucial observation is an immediate effect of the actuation at the sensor location, even though the sensor is far from the actuator location. After a rapid decay and a transient response, the signal settles into a periodic pattern which eventually decays exponentially.

Since all frequencies are excited by an initial impulse, a convenient and alternative way of expressing the input-output behaviour of a linear system is to switch to the frequency domain. The amplitude of the transfer function, which is defined as the Fourier transform of the impulse response, is displayed in figure $4(b)$. This graph represents the amplitude part of a standard-type gain-phase plot (e.g. a Bode plot); Since we use an impulse function on a causal system with $m=0$ for all times $t<0$, the transfer function is simply given by $H(\omega)=\int_{0}^{\infty} \mathrm{e}^{-\mathrm{i} \omega t} m(t) \mathrm{d} t$. We recover a preferred frequency around $\omega=4.6$ which corresponds to the periodic signal observed in figure $4(a)$.

In the following sections, we will use various bases to produce reduced models for the stable subspace dynamics; in particular, we are interested in the dimensionality of a particular basis to approximate the full transfer function to a prescribed degree of accuracy. The discrepancy between reduced and full transfer function will be quantified using the $\mathscr{H}_{\infty}$ norm defined as

$$
\|G(\omega)\|_{\infty}=\sup _{\omega}|G(\omega)| .
$$

The choice of this norm has been motivated by the availability of theoretical bounds, as will be shown later. Nevertheless, the $\mathscr{H}_{2}$ norm has also been computed for each of the following cases, and the results were found in qualitative agreement with the $\mathscr{H}_{\infty}$ norm. The computation of the transfer function for the reduced model is obtained 

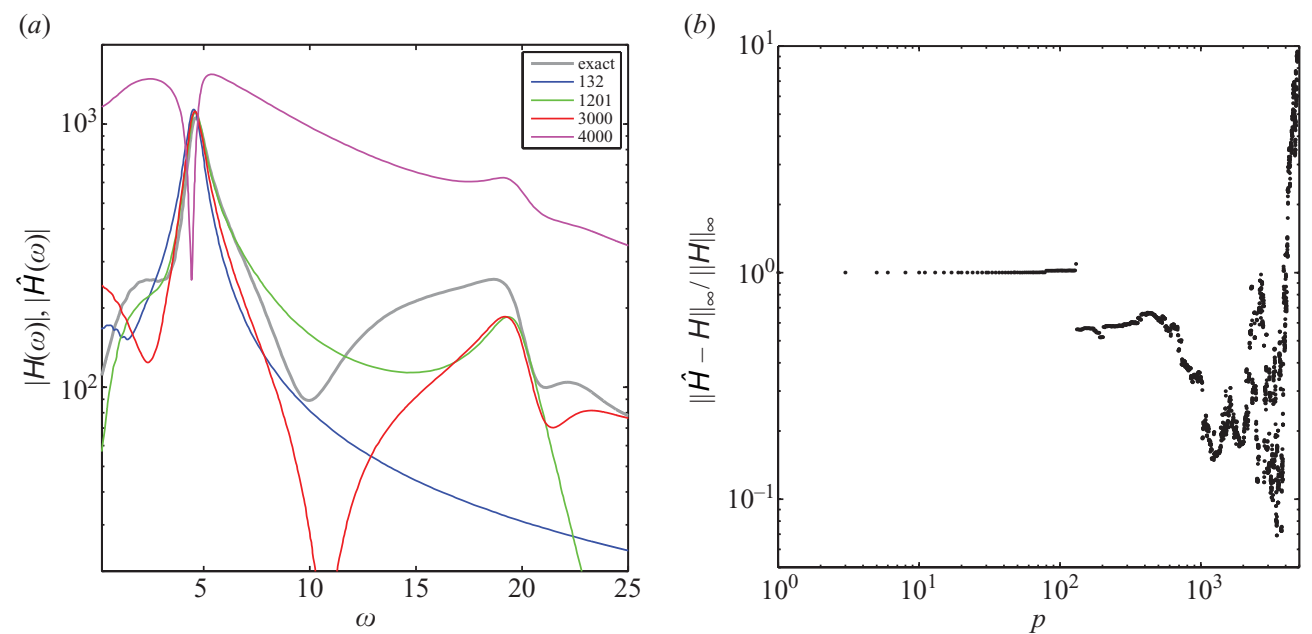

FIGURE 5. (a) Comparison of transfer function of the reduced-order model to the exact transfer function for a projection basis consisting of $p=132,1201,3000,4000$ stable global modes. $(b)$ Relative $\mathscr{H}_{\infty}$-error norm as a function of the number $p$ of included stable global modes.

using the equivalent denotation $\hat{H}(\omega)=\hat{\boldsymbol{M}}_{s}\left(\mathbf{i} \omega \hat{\boldsymbol{I}}-\hat{\boldsymbol{A}}_{s}\right)^{-1} \hat{\boldsymbol{C}}_{s}$ where the quantities with refer to the reduced matrices.

\subsection{Reduced-order modelling using global modes}

\subsubsection{Transfer function and error norm}

We start by considering stable global modes as a basis for computing a reducedorder model; in particular, a stable subspace consisting of $p$ stable global modes, ranked by decaying amplification rate (see Åkervik et al. 2007 and Ehrenstein \& Gallaire 2008), are taken. The transfer functions corresponding to reduced models based on $p=132,1201,3000$ and 4000 global modes are displayed in figure 5(a) and compared to the transfer function of the full system. It is evident that none of the reduced models is able to approximate the exact transfer function to a satisfactory degree of accuracy. Moreover, although the curves based on $p=132, p=1201$ and $p=3000$ accurately capture the peak around $\omega=5$, the reduced-order model based on $p=4000$ modes fails entirely, and it appears that taking additional stable modes does not necessarily improve the quality of the reduced-order model. This is also visible in figure $5(b)$ where the $\mathscr{H}_{\infty}$-error $\|\hat{H}-H\|_{\infty} /\|H\|_{\infty}$ is plotted versus the number of stable eigenmodes accounted for in the reduced-order model. For reduced models including up to 100 modes, the error remains at order one. After the 132 th mode ( $E_{2}$ in table 1$)$ has been included in the basis, the error decreases abruptly, thus indicating that this mode is important to represent the correct input-output behaviour. As more global modes are added, the $\mathscr{H}_{\infty}$-error decreases again, starts to oscillates and finally increases substantially. On the whole, the quality of the reduced-order model is never satisfactory and generally becomes worse as additional modes are included. Nevertheless, the inclusion of a few particular modes results in a significantly drop in the error norm; these modes warrant some further investigation as to their contribution to the input-output behaviour of the system. Specifically, a criterion will be derived in the next section that facilitates the ranking of stable global modes according to their importance in representing the transfer function. 


\subsubsection{Criterion to select stable global modes}

The erratic convergence behaviour of the transfer function of a reduced-order system based on global modes to the transfer function of the full system portends the fact that the choice of which stable global modes to include in the reduced-order model of the estimator is far from trivial. Nevertheless, a theoretical criterion that guides this choice can be derived (see also Bagheri et al. 2009b). To this end, let us first recall the equations that govern the dynamics of the reduced-order model:

$$
\begin{aligned}
\frac{\mathrm{d} \hat{\boldsymbol{X}}}{\mathrm{d} t} & =\hat{\boldsymbol{\Lambda}} \hat{\boldsymbol{X}}+\hat{\boldsymbol{C}}_{c}, \\
\hat{m} & =\hat{\boldsymbol{M}} \hat{\boldsymbol{X}} .
\end{aligned}
$$

For simplicity and without loss of generality, we consider a particular control law in the form

$$
c(t)=\cos (\omega t)
$$

where $\omega$ is a given (real) frequency. We then determine the forced response of the dynamical system expressed in terms of the measurement and omitting the initial transient response. We obtain

$$
\hat{m}=\frac{1}{2} \hat{\boldsymbol{M}}(\mathrm{i} \omega \hat{\boldsymbol{I}}-\hat{\boldsymbol{\Lambda}})^{-1} \hat{\mathbf{C}} \exp (\mathrm{i} \omega t)+\frac{1}{2} \hat{\boldsymbol{M}}(-\mathrm{i} \omega \hat{\boldsymbol{I}}-\hat{\boldsymbol{\Lambda}})^{-1} \hat{\mathbf{C}} \exp (-\mathrm{i} \omega t)
$$

from which we may extract the contribution of the $i$ th global mode as

$$
\hat{m}_{i}=\frac{1}{2} \frac{\hat{\boldsymbol{M}}_{i} \hat{\boldsymbol{C}}_{i}}{\mathrm{i} \omega-\hat{\boldsymbol{\Lambda}}_{i}} \exp (\mathrm{i} \omega t)+\frac{1}{2} \frac{\hat{\boldsymbol{M}}_{i} \hat{\boldsymbol{C}}_{i}}{-\mathrm{i} \omega-\hat{\boldsymbol{\Lambda}}_{i}} \exp (-\mathrm{i} \omega t) .
$$

If the $i$ th eigenvalue has a non-zero imaginary part, we get $\hat{\mathbf{M}}_{i+1}=\hat{\mathbf{M}}_{i}^{*}, \hat{\boldsymbol{C}}_{i+1}=\hat{\mathbf{C}}_{i}^{*}$ and $\boldsymbol{\Lambda}_{i+1}=\boldsymbol{\Lambda}_{i}^{*}$. It then follows that $\hat{m}_{i+1}=\hat{m}_{i}^{*}$ and further

$$
m_{i}+m_{i+1}=\left|\frac{\hat{\boldsymbol{M}}_{i} \hat{\boldsymbol{C}}_{i}}{\mathrm{i} \omega-\hat{\boldsymbol{\Lambda}_{i}}}\right| \cos (\omega t+\psi)+\left|\frac{\hat{\boldsymbol{M}}_{i} \hat{\boldsymbol{C}}_{i}}{-\mathrm{i} \omega-\hat{\boldsymbol{\Lambda}_{i}}}\right| \cos (-\omega t+\boldsymbol{\xi}) .
$$

The forced response is the sum of waves of frequency $\omega$. Maximizing over all forcing frequencies $\omega$, we can see that the contribution of the $i$ th eigenmode is proportional to the quantity (see also Antoulas 2005, p. 282 and Bagheri et al. 2009b):

$$
\Gamma_{i}=\frac{\left|\hat{\boldsymbol{M}}_{i}\right|\left|\hat{\boldsymbol{C}}_{i}\right|}{\left|\operatorname{Re}\left(\hat{\boldsymbol{\Lambda}}_{i}\right)\right|}
$$

This criterion takes into account three different physical variables to quantify if a stable global mode should or should not be retained in the reduced-order model basis of the estimator: $(a)$ the measurement coefficient $\hat{\boldsymbol{M}}_{i}$, which is closely related to the observability of the respective global mode, $(b)$ the control coefficient $\hat{\boldsymbol{c}}_{i}$, a measure of controllability of the associated global mode and (c) the decay rate $\left|\operatorname{Re}\left(\boldsymbol{\Lambda}_{i}\right)\right|$ of the global mode. In short, the criterion based on $\Gamma_{i}$ selects global modes that are observable, controllable and weakly damped. It is noteworthy that Lee et al. (2001) used a similar technique for a reduced model by selecting modes that are equally controllable and observable.

This analysis should validate, in some sense, the choice of least stable global modes as adopted by Åkervik et al. (2007). Indeed, if the measurement and control coefficients of all global modes were bounded (an assumption that will be proven wrong below), 

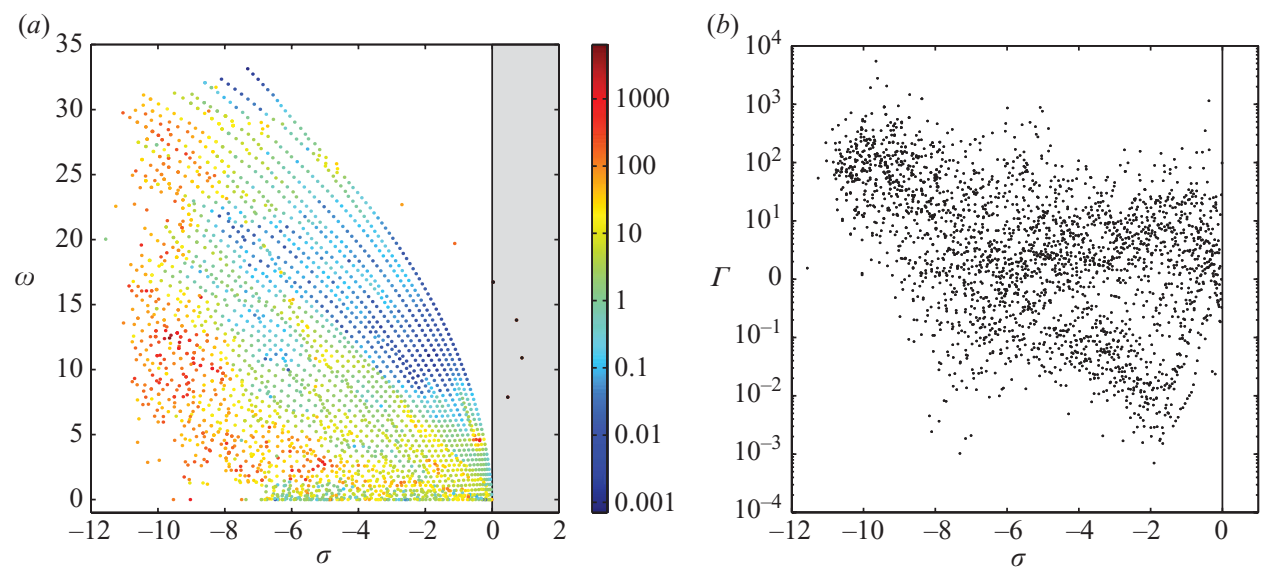

FIGURE 6. (a) Global spectrum of flow over an open square cavity at $R e=7500$ colour-coded by $\Gamma_{i}$, a measure of each global mode's contribution to the input-output relation (see (5.8) and text for explanation). (b) Criterion $\Gamma_{i}$ versus the each mode's amplification rate.

then $\Gamma_{i}$ would decay as $1 /\left|\operatorname{Re}\left(\boldsymbol{\Lambda}_{i}\right)\right|$ as we move to larger decay rates $\left|\operatorname{Re}\left(\boldsymbol{\Lambda}_{i}\right)\right|$. This would then justify neglecting highly damped global modes in the reduced-order basis of the estimator. But the choice of included global modes, made purely on their decay rate, does not yield satisfactory results since the notion of controllability and observability of the global modes has not been taken into account.

For our present case of open flow over a square cavity, we observe that $\Gamma_{i}$ does not decrease with the decay rate $\left|\operatorname{Re}\left(\hat{\boldsymbol{\Lambda}}_{i}\right)\right|$ of the global modes. In figure $6(a)$, which shows the global spectrum in the $(\sigma, \omega)$ plane, the colouring of the eigenvalues corresponds to $\Gamma_{i}$. The eigenvalues that appear in warm (cold) colours display high (low) values of $\Gamma$; a logarithmic colour map has been used here and $\Gamma_{i}$ is meaningful only for damped eigenmodes. We observe that some of the highest values of $\Gamma$ appear for strongly damped eigenmodes; moreover, the number of eigenmodes with high values of $\Gamma$ appears to increase as the damping increases since nearly all yellowand red-coloured eigenvalues appear on the left side of the figure. In figure $6(b)$, the same information is plotted in a different form: the horizontal axis represents the amplification rate $\sigma$ of each eigenvalue while the vertical axis represents its value of $\Gamma$ (on a logarithmic scale). It is clear that $\Gamma$ does not decrease for highly damped eigenvalues and that more and more eigenvalues with high $\Gamma$ appear as the damping rate $-\sigma$ increases. Since no theoretical argument can be made for an eventual decrease of $\Gamma$ as the damping rate increases, we conclude that the damping rate is a poor and inappropriate measure for the judicious inclusion of global modes into the reduced-order model or for the truncation of the expansion basis. We also want to remind ourselves that numerical difficulties stemming from the non-normal nature of the problem prevented us from computing converged global modes beyond the damping rates shown in the above figures. Furthermore, reduced-order models with more than about 100 degrees of freedom quickly become untractable and impractical for closed-loop control efforts.

The divergence of $\Gamma$ with the damping rate $-\sigma$ warrants a more thorough investigation. We recall from the definition of $\Gamma$ given in (5.8) that it consists of the product of the control coefficient $\hat{\boldsymbol{C}}_{i}$ and the measurement coefficient $\hat{\boldsymbol{M}}_{i}$ divided by the damping rate. In figures $7(a)$ and $7(b)$ we present, for all converged 
(a)

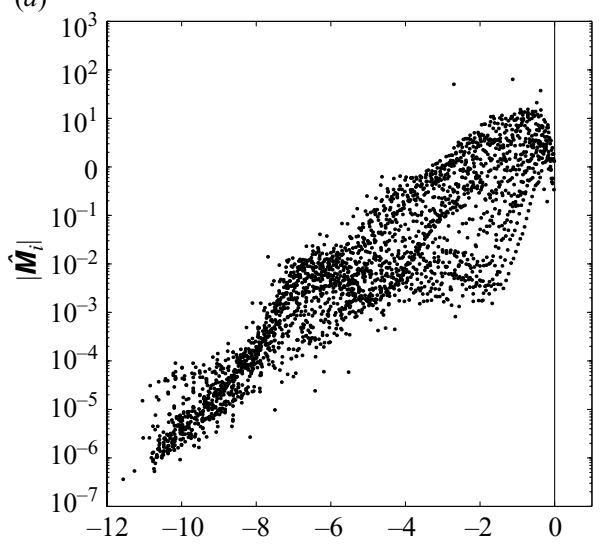

(c)

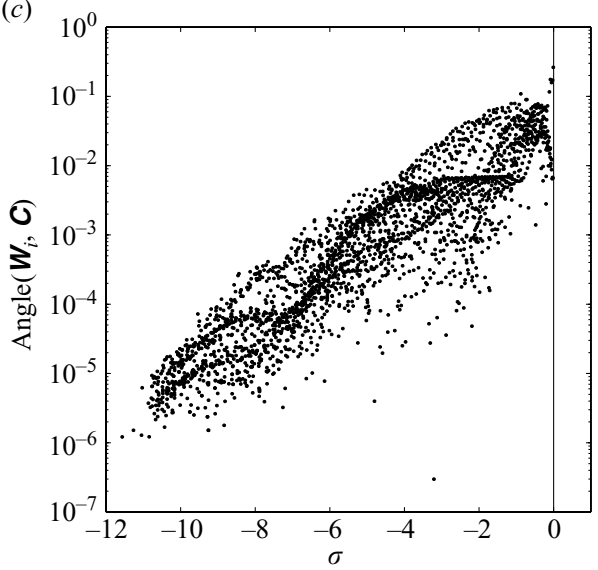

(b)

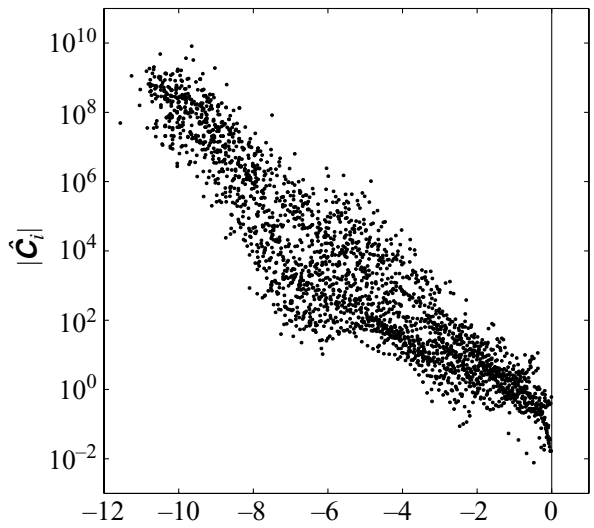

(d)

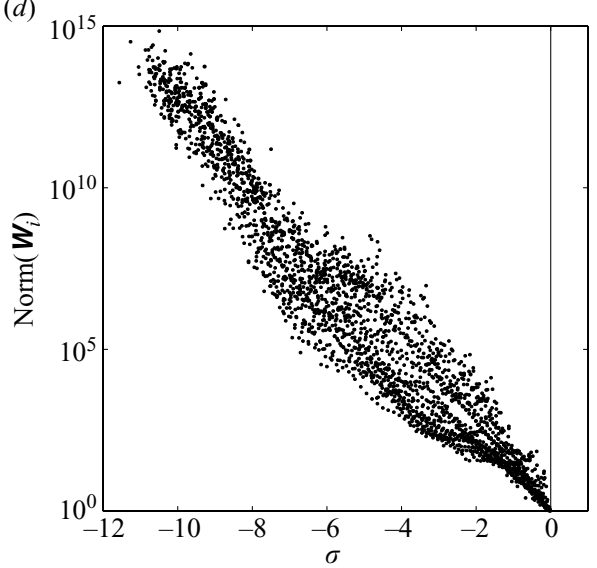

FIGURE 7. Breakup of criterion $\Gamma_{i}$ into its individual components. (a) Measurement coefficient $\hat{\boldsymbol{M}}_{i}$ versus the amplification rate, and $(b)$ control coefficient $\hat{\boldsymbol{C}}_{i}$ versus the amplification rate. The control coefficient $\hat{\boldsymbol{C}}_{i}$ is further factored according to (5.9) into $(c)$ the angle between adjoint global mode $\boldsymbol{W}_{i}$ and control function $\boldsymbol{C}$, and $(d)$ the norm of the adjoint mode $\boldsymbol{W}_{i}$ which are displayed versus the amplification rate of the corresponding global mode.

eigenvalues, the modulus of the measurement coefficient and the control coefficient as a function of the amplification rate. Figure 7(a) reveals that modes with higher damping rates have a lower observability. This tendency alone would suggest that based on observability we would be justified in excluding global modes with higher damping. An opposite trend can be observed when controllability is considered: in this case, controllability increases with increasing damping rates. The disadvantageous behaviour of the composite coefficient $\Gamma$ can thus be traced back to the controllability coefficient defined as $\hat{\boldsymbol{C}}_{i}=\boldsymbol{W}_{i}^{*} \mathbf{Q C}$. We note that

$$
\left|\hat{\boldsymbol{C}}_{i}\right|=\sqrt{\mathbf{C}^{*} \boldsymbol{Q C}} \underbrace{\frac{\left|\boldsymbol{W}_{i}^{*} \mathbf{Q C}\right|}{\sqrt{\boldsymbol{W}_{i}^{*} \boldsymbol{Q} \boldsymbol{W}_{i}} \sqrt{\mathbf{C}^{*} \mathbf{Q C}}}}_{\text {angle }\left(\boldsymbol{W}_{i}, \boldsymbol{C}\right)} \underbrace{\sqrt{\boldsymbol{W}_{i}^{*} \boldsymbol{Q} \boldsymbol{W}_{i}}}_{\text {norm }\left(\boldsymbol{W}_{i}\right)} .
$$

The second term on the right-hand side of the above expression describes the angle between the adjoint global mode $\boldsymbol{W}_{i}$ and the control function $\boldsymbol{C}$ while the last 
term on the right-hand side denotes the norm of the adjoint mode $\boldsymbol{W}_{i}$, which may be interpreted as a measure of non-normality of the global mode $\boldsymbol{V}_{i}$. These two quantities have been plotted versus the amplification rate $\sigma$ of each global mode in figures $7(c)$ and $7(d)$, respectively. These two figures show that the non-normality (figure $7 d$ ), rather than the angle term (figure $7 c$ ), is responsible for the overall lack of convergence of $\Gamma$ with respect to the damping rate $-\sigma$. This non-normality of the damped global mode basis is linked to physical properties of the base flow. Non-normality has also been identified by Lauga \& Bewley (2003) as the reason for loss of stabilizability in their study of the controlled linear Ginzburg-Landau model.

To summarize this section, global modes have been used as a projection basis to capture the input-output behaviour of the stable subspace. Selecting global modes according to either their damping rate or their contribution to the input-output behaviour produced disappointing results as no satisfactory agreement between the reduced and the full transfer function could be obtained. For this reason, other expansion bases to construct a reduced-order model have to be considered.

\subsection{Reduced-order modelling using BPOD modes}

A powerful technique, commonly applied in control theory and known as balanced truncation, allows us to reduce the stable part of the linear system while optimally preserving its input-output behaviour. The key idea is to compute, rank and select modes that are equally observable and controllable.

We will see in \$5.3.2 that the computational effort to compute these balanced modes is rather high for systems of moderate size and quickly becomes unaffordable for systems of large size and realistic complexity. Instead, we will compute an approximation of the balanced modes by an algorithm referred to as balanced proper orthogonal decomposition (BPOD) which remains tractable even for very large systems (see Rowley 2005).

Before dealing with balanced truncation, we will first introduce and analyse the concept of controllability and observability Gramians which will lay the foundation and provide the necessary background for the derivation and application of the exact and approximate balancing transformation. We rewrite (5.1) in the classical state-space formulation (see $\S$ A.1 of appendix):

$$
\begin{aligned}
\frac{\mathrm{d} \boldsymbol{X}_{1}}{\mathrm{~d} t} & =\boldsymbol{A}_{1} \boldsymbol{X}_{1}+\boldsymbol{C}_{1} c, \\
m & =\boldsymbol{M}_{1} \boldsymbol{X}_{1},
\end{aligned}
$$

which governs the dynamics of the velocity field $\boldsymbol{X}_{1}$. In what follows, the subscript ${ }_{1}$ will refer to quantities that consist of the velocity components only. We note that the scalar product restricted to the velocity field is written accordingly as $\boldsymbol{X}_{1}^{*} \boldsymbol{Q}_{1} \boldsymbol{X}_{1}$.

\subsubsection{Controllability and observability Gramians}

A specific state is deemed controllable if there exists a control law $c(t)$ which is able to modify the flow from any state towards this specific state. For controllable states, the notion of controllability then quantifies how easy (or difficult) the state can be reached from any state. Mathematically this is expressed in terms of the controllability Gramian $\mathbf{G}_{C}$ defined as

$$
\boldsymbol{G}_{C}=\int_{0}^{\infty} \mathrm{e}^{\boldsymbol{A}_{1} t} \boldsymbol{C}_{1} \boldsymbol{C}_{1}^{*} \mathrm{e}^{\boldsymbol{A}_{1}^{*} t} \mathrm{~d} t,
$$


which is a convergent integral as $t \rightarrow \infty$ since $\boldsymbol{A}_{1}$ is stable. The expression $\boldsymbol{X}_{1}^{*} \boldsymbol{G}_{C}^{-1} \boldsymbol{X}_{1}$ can be interpreted as the minimum control energy expended over an infinite time horizon, i.e. $\int_{0}^{\infty} c(t)^{2} \mathrm{~d} t$, to drive the system from the initial zero state to the state $\boldsymbol{X}_{1}$ (see more details in $\S$ A.2 of appendix). Using the snapshot technique introduced by Rowley (2005) the controllability Gramian may be factored as

$$
\mathbf{G}_{C}=\boldsymbol{T}_{1} \boldsymbol{T}_{1}^{*},
$$

where $\boldsymbol{T}_{1}$ stands for the matrix containing $n_{d}$ rescaled snapshots arising from a DNS initialized by the control matrix $\mathbf{C}$ at $t=0$ (see $\S$ A.2). Note that this is the same simulation as the impulse response presented in $\S 5.1$.

As controllability quantifies the amount of effort it takes to manipulate a particular flow state, observability measures how easy (or difficult) a given flow state can be detected by the sensors. At the sensor location almost unobservable flow states leave hardly any footprint behind and are thus nearly 'invisible' to our measurement efforts. The mathematical quantity that enables measuring the observability of a given flow state is the observability Gramian which is defined as

$$
\boldsymbol{G}_{O}=\int_{0}^{\infty} \mathrm{e}^{\boldsymbol{A}_{1}^{*} t} \boldsymbol{M}_{1}^{*} \boldsymbol{M}_{1} \mathrm{e}^{\boldsymbol{A}_{1} t} \mathrm{~d} t
$$

The expression $\boldsymbol{X}_{1}^{*} \boldsymbol{G}_{O} \boldsymbol{X}_{1}$ represents the maximum energy $\int_{0}^{\infty} m^{2}(t) \mathrm{d} t$ produced by observing the output of the initial state $\boldsymbol{X}_{1}$ (more details are given in $\S$ A.3). Again following Rowley (2005) the observability Gramian may be factored according to

$$
\mathbf{G}_{O}=\boldsymbol{Q}_{1} \boldsymbol{U}_{1} \boldsymbol{U}_{1}^{*} \mathbf{Q}_{1}
$$

where $\boldsymbol{U}_{1}$ denotes the matrix containing $n_{a}$ snapshots arising from the simulation of the adjoint problem initialized by the measurement matrix $\boldsymbol{M}$ at $t=0$ (see $\S \mathrm{A} .3$ ). It should be mentioned at this stage that the above-described technique requires the knowledge of the adjoint state. For experimental applications, this approach may thus become inapplicable. However, recent attempts to determine balanced modes without having to resort to adjoint information have been reported by Or \& Speyer (2008) and Ma, Ahuja \& Rowley (2009).

\subsubsection{Balancing transformation using the snapshot method}

Since both controllability and observability are important when choosing a reduction basis that preserves the input-output relation, a technique has to be applied that extracts flow fields with equal emphasis on either property. This technique is referred to as balancing. Mathematically, it is equivalent to finding a transformation basis in which the Gramians $\boldsymbol{G}_{C}$ and $\boldsymbol{G}_{O}$ appear diagonal and equal (see Zhou et al. 2002). This basis, denoted by $\boldsymbol{J}$, and its associated adjoint basis, denoted by $\boldsymbol{K}$, satisfy the bi-orthogonality relation

$$
\boldsymbol{K}^{*} \mathbf{Q} \mathbf{J}=\boldsymbol{I}
$$

where $\boldsymbol{Q}$ takes into account the finite-element discretization by adding a weight matrix to the standard inner product. The established procedure of finding the balancing transformation consists of computing the Gramians (see $\S$ A.2 and $\S$ A.3) followed by an eigenvalue decomposition of one of the following products

$$
\begin{aligned}
\boldsymbol{G}_{C} \boldsymbol{G}_{O} \boldsymbol{J}_{1} & =\boldsymbol{J}_{1} \boldsymbol{\Sigma}^{2}, \\
\boldsymbol{G}_{O} \boldsymbol{G}_{C}\left(\boldsymbol{Q}_{1} \boldsymbol{K}_{1}\right) & =\left(\boldsymbol{Q}_{1} \boldsymbol{K}_{1}\right) \boldsymbol{\Sigma}^{2}
\end{aligned}
$$


These eigenvalue problems yield the same eigenvalues, since the transpose of the matrix $\boldsymbol{G}_{C} \boldsymbol{G}_{O}$ is simply $\boldsymbol{G}_{O} \boldsymbol{G}_{C}$. The diagonal matrix $\boldsymbol{\Sigma}$ contains the Hankel singular values. By definition, the Hankel singular values are the non-zero singular values of the Hankel operator associated with our linear system.

Solving the Lyapunov equations and the eigenvalue problem for the balancing transformation, however, is computationally not feasible. It has been shown (Rowley 2005) that a good approximation of this transformation can be obtained while entirely avoiding the explicit computation of the Gramians. The associated technique relies on the decomposition of the Gramians in the form of (5.12) and (5.14).

A singular value decomposition (SVD) of the matrix product $\boldsymbol{U}_{1}^{*} \boldsymbol{Q}_{1} \boldsymbol{T}_{1}=\boldsymbol{U}^{*} \boldsymbol{Q} \boldsymbol{T}$, which is of dimension $n_{a} \times n_{d}$, results in

$$
\begin{aligned}
\boldsymbol{U}_{1}^{*} \boldsymbol{Q}_{1} \boldsymbol{T}_{1} & =\boldsymbol{U}^{\prime} \boldsymbol{\Sigma} \boldsymbol{T}^{\prime *}, \\
\boldsymbol{U}^{\prime *} \boldsymbol{U}^{\prime} & =\boldsymbol{I}, \\
\boldsymbol{T}^{\prime *} \boldsymbol{T}^{\prime} & =\boldsymbol{I},
\end{aligned}
$$

where $\boldsymbol{T}^{\prime}, \boldsymbol{U}^{\prime}$ and $\boldsymbol{\Sigma}$ are of dimension $n_{d} \times n_{d}, n_{a} \times n_{a}$ and $n_{a} \times n_{d}$, respectively. In a final step, the bi-orthogonal balancing bases given by $\boldsymbol{J}$ and $\boldsymbol{K}$ in (5.16) are found as

$$
\begin{aligned}
& \boldsymbol{J}=\boldsymbol{T} \boldsymbol{T}^{\prime} \boldsymbol{\Sigma}^{-1 / 2}, \\
& \boldsymbol{K}=\boldsymbol{U} \boldsymbol{U}^{\prime} \boldsymbol{\Sigma}^{-1 / 2}
\end{aligned}
$$

It is easily confirmed that the bi-orthogonality condition $\boldsymbol{K}^{*} \mathbf{Q} \boldsymbol{J}=\boldsymbol{I}$ is satisfied. We verify that

$$
\begin{aligned}
\left(\boldsymbol{Q}_{1} \boldsymbol{K}_{1}\right)^{*} \boldsymbol{G}_{C}\left(\boldsymbol{Q}_{1} \boldsymbol{K}_{1}\right) & =\boldsymbol{\Sigma}, \\
\boldsymbol{J}_{1}^{*} \boldsymbol{G}_{O} \boldsymbol{J}_{1} & =\boldsymbol{\Sigma} .
\end{aligned}
$$

In Rowley (2005) it is shown that this implies that the columns of $\boldsymbol{J}_{1}$ form the first columns of the balancing transformation and the columns of $\boldsymbol{Q}_{1} \boldsymbol{K}_{1}$ constitute the first columns of the inverse transformation.

We conclude this section by emphasizing the fact that our initial condition $(\boldsymbol{M})$ for the adjoint simulation to obtain the approximate observability Gramian is different from the one used by Rowley (2005) where the so-called output-projection technique has been applied. This technique relies on first defining the entire state vector as the output of the linear system. This in turn would call for one simulation for each degree of freedom, a requirement that of course cannot be met. To nevertheless capture the entire state in an optimal way, a projection onto POD modes is applied. The initial conditions for the adjoint simulations are taken as these POD modes. As a result, the input-output behaviour captured by their BPOD modes is between the actuator (input) and the dynamics described by the POD modes. This technique may yield a good estimation of the interaction of the actuator with the most energetic structures.

If an estimate of the flow state using the measurement matrix $\boldsymbol{M}$ is needed, an increased number of POD modes may be necessary to reconstruct an accurate measurement signal, since POD modes, by design, are not particularly efficient in capturing information near the sensor. For instance, in Rowley et al. (2008) 20 POD modes were needed which implies an equal number of adjoint simulations, a rather costly requirement.

In our case, the output of our system is solely the measurement signal from the sensor. The flow states which are observable from this sensor are given as the result of an adjoint simulation. For this reason, only one adjoint simulation (with the measurement matrix as the initial condition) is necessary. The apparent drawback, 
namely that our reduced model may not accurately capture the dynamics in the rest of the domain but merely describe the states excited by the actuator and detected by the sensor, is inconsequential since our primary objective is to control the flow over the cavity by a reduced-order model that is as low-dimensional as possible, and for this objective the input-output behaviour is the only quantity of importance.

\subsubsection{Results}

Both the direct and adjoint simulations are integrated up to $T=16$ using a secondorder accurate scheme with a fixed time step of $\Delta t=2 \times 10^{-4}$ in order to produce and gather the snapshots. The direct simulation is identical to the one for extracting the impulse response (see § 5.1) and illustrates the effect of the control on the stable part of the flow. The adjoint simulation (defined in (A 17)) is initialized with the matrix $\boldsymbol{M}$ which represents a unit measurement signal. As was the case for the direct simulation, a projection onto the stable subspace is necessary which was accomplished in an analogous manner. The adjoint simulation then gives us information on which flow structures will be most detectable by the sensor.

Balanced modes forming the basis for the balancing transformation are computed from a sequence of snapshots that are equispaced at an interval of $80 \Delta t=1.6 \times 10^{-2}$. With this parameter setting we produce $n_{d}=n_{a}=1001$ snapshots for each the direct and adjoint simulation.

In figure $8(a)$ the singular values (SV) $\boldsymbol{\Sigma}_{i i}$ from the singular value decomposition of $\boldsymbol{U}_{1}^{*} \boldsymbol{Q}_{1} \boldsymbol{T}_{1}$ (see (5.17)) are shown. These singular or Hankel values provide a measure of how controllable (and observable) the corresponding balanced modes are. This information can then be used to decide on a truncation point and thus on the size of the reduced-order model. Significant drops in the Hankel values are commonly used to justify truncation of the balancing basis.

In figure 8 the balanced modes corresponding to the first, second, ninth and thirteenth singular value are displayed. The first two modes show a similar structure: vortices are present on the shear-layer, and the effect of actuation is clearly visible. The pairwise occurrence of balanced modes with the displayed spatial structure is linked to the representation of travelling structures by the superposition of these modes. Both modes show an increased amplitude near the actuator (at the upstream edge of the cavity). The ninth balanced mode is located mainly inside the cavity with a structure reminiscent of the least stable global mode (see figure $2 d$ ). The 13th BPOD mode again shows structures inside the cavity as well as in the boundary layer. Near the point of actuation a small but noticeable amplitude is present.

\subsubsection{Transfer function and error norm}

Next, we consider reduced-order models $\left(\hat{\boldsymbol{A}}_{s}, \hat{\boldsymbol{C}}_{s}, \hat{\boldsymbol{M}}_{s}\right)$ based on $p$ BPOD modes where the modes are ranked by decreasing Hankel values. The transfer function corresponding to reduced-order models based on $p=2,6,8$ and 10 modes are compared to the exact transfer function in figure $9(a)$. Contrary to the reduced-order models based on global modes, the exact transfer function is very well approximated even for a moderate number $p$ of modes. When only two balanced modes are considered, the main peak is already well-captured. Figure $9(a)$ is meant to convey the convergence behaviour of reduced-order transfer functions towards the exact one for a particular number $p$ of BPOD modes. As the number of modes further increases, the approximate transfer function converges rapidly to the exact one. For $p=13$ modes (not shown in figure $9(a)$ ), the curves for the approximate and exact transfer function coincide to plotting accuracy; the dynamics of the stable subspace 
(b)
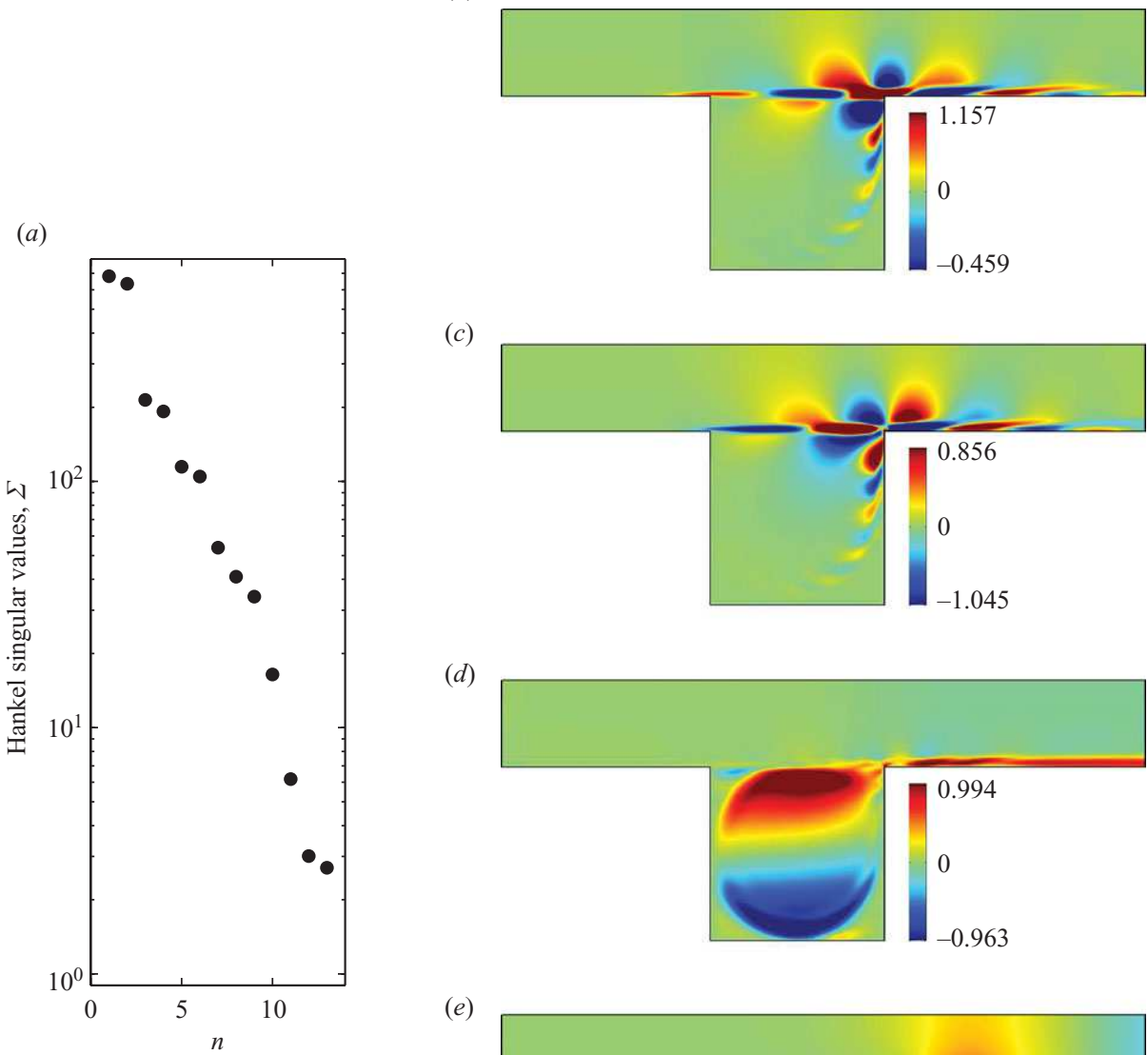

(c)

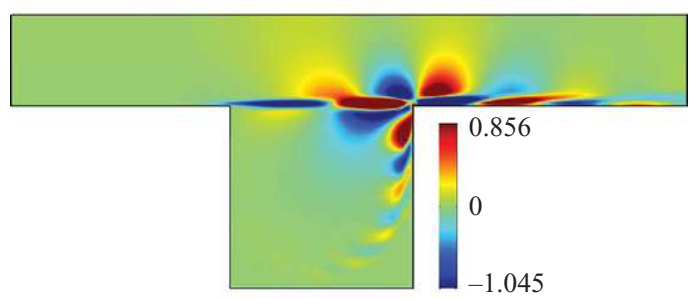

(d)

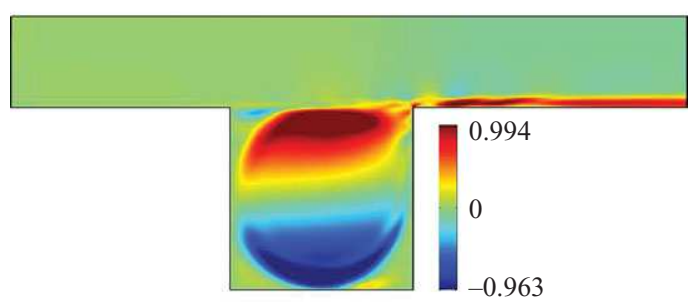

(e)

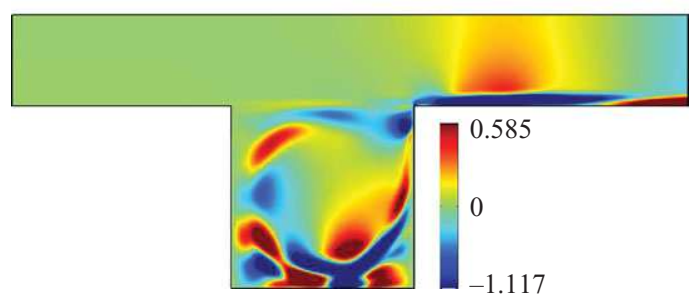

Figure 8. (a) Hankel singular values for flow over a square cavity for $R e=7500$. $(b-e)$ Balanced proper orthogonal decomposition (BPOD) modes. (b) First, $(c)$ second, $(d)$ ninth and $(e)$ thirteenth mode, visualized by the streamwise velocity.

and its projection onto 13 BPOD modes produces virtually the same input-output behaviour.

Bounds on the discrepancy between the approximate and exact transfer function are readily available for balanced truncation. An upper bound for the infinity norm (maximum error) of the transfer function error is given by twice the sum of the neglected Hankel values (see Antoulas 2005). We have

$$
\|\hat{H}-H\|_{\infty} \leqslant 2 \sum_{j=r+1}^{n_{1}} \Sigma_{j j},
$$



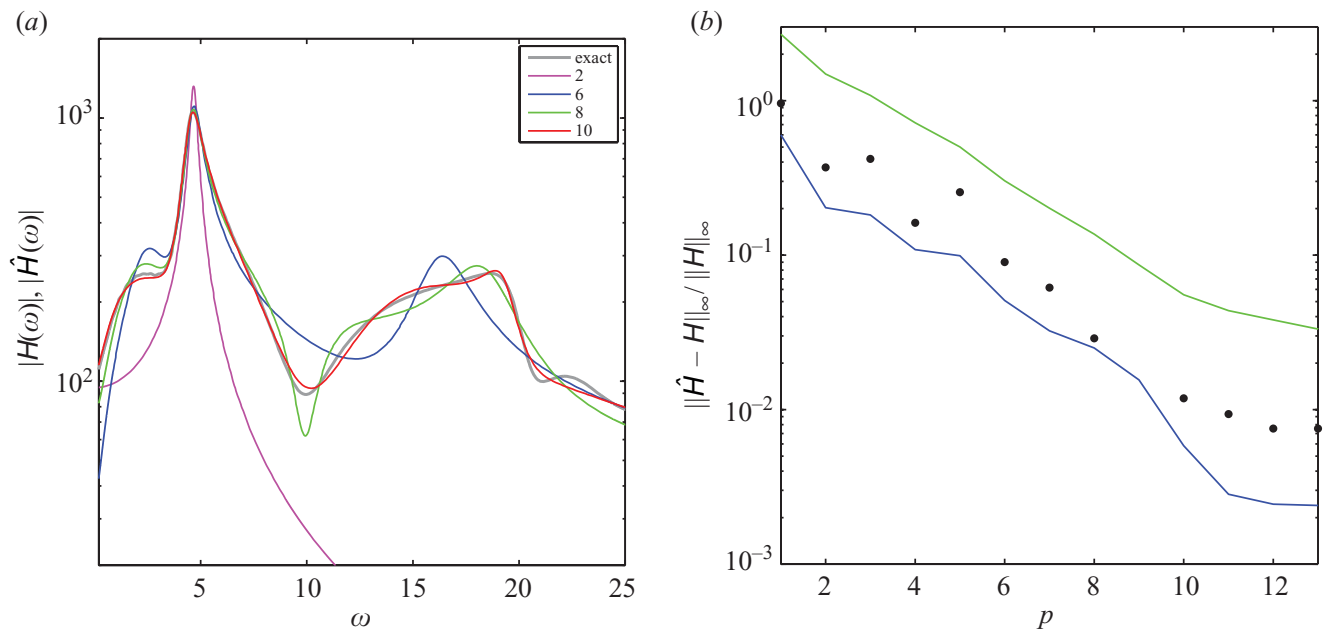

FIGURE 9. (a) Comparison of transfer function of the reduced-order model to the exact transfer function for a projection basis consisting of $p=2,6,8,10$ balanced modes. $(b)$ Relative $\mathscr{H}_{\infty}$-error norm as a function of the number $p$ of included balanced modes, together with an upper (green line) and lower bound (blue line) on the error.

where $H$ and $n_{1}$ are the transfer function and dimension of the full system, respectively, whereas $\hat{H}$ and $r$ are the equivalent quantities for the reduced-order model. Comparisons made in Rowley (2005), Ilak \& Rowley (2008) and Bagheri et al. $(2009 b)$ suggest that the error committed by approximating the true Gramians by a series of discrete-time snapshots is very close to balanced truncation. A lower bound on the maximum error also exists for any reduced-order model. It can be stated as

$$
\|\hat{H}-H\|_{\infty}>\Sigma_{r+1} \text {. }
$$

In figure $9(b)$, the $\mathscr{H}_{\infty}$ error is displayed together with the two error bounds discussed above. For all cases, as required, the error falls between the upper and lower bounds for balanced truncation.

\subsubsection{The eigenvalues of the reduced-order model based on BPOD modes}

The ability of reduced-order models based on BPOD modes to capture the inputoutput behaviour, as demonstrated above by comparing the approximate and exact transfer function, raises the question of which parts of the full global spectrum contribute to this input-output relation. In answering this question we will establish a link between balanced truncation and the criterion (5.8), introduced in $\$ 5.2 .2$, that quantifies the contribution of selected global modes to the input-output behaviour.

In figure 10 the eigenvalues of the reduced-order system matrix $\hat{\boldsymbol{A}}_{p, p}$ based on $p=13$ BPOD modes are superimposed on the full global spectrum of the cavity which is, in addition, colour-coded by the criterion $\Gamma_{i}$ (same as figure 6). As the selection criterion $\Gamma_{i}$ is only valid for the stable global modes, we will restrict ourselves to the stable part of the spectrum.

We first notice that the eigenvalues of the BPOD-reduced system are bounded by the global spectrum. This is expected since the BPOD modes approximate the subspace spanned by the stable global modes. Furthermore, varying the order of the BPOD-reduced model does not have any influence on certain eigenvalues of the reduced-order model (not shown here). These eigenvalues, labelled $M_{1}, M_{2}$, and 


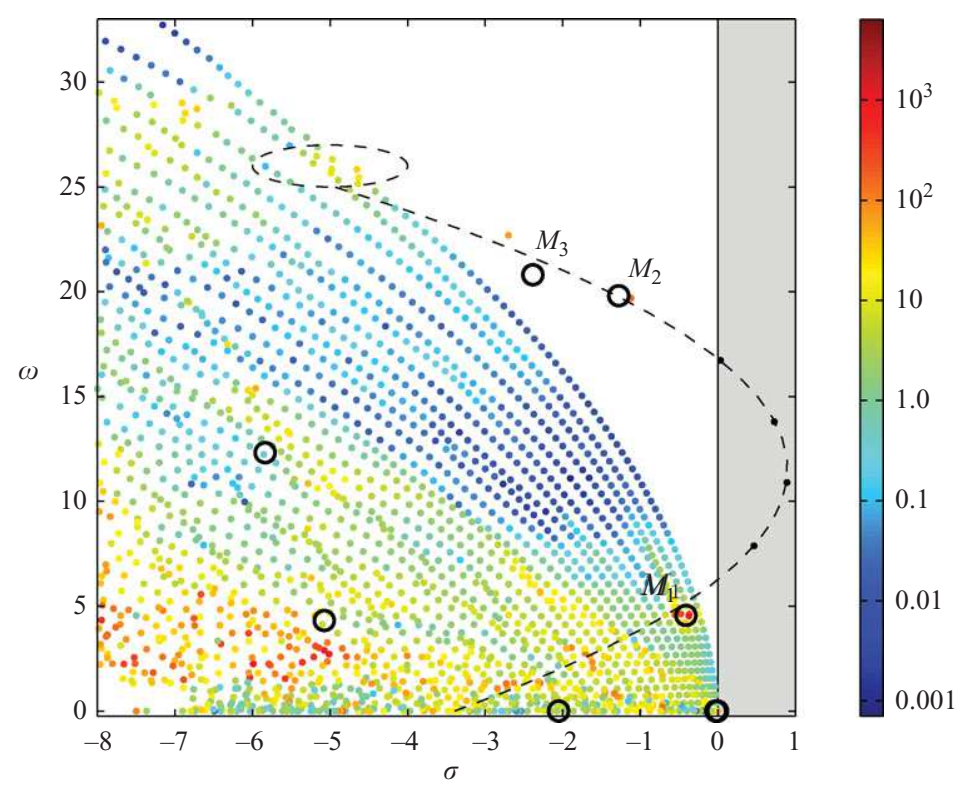

FIGURE 10. Eigenvalues of the reduced matrix $\hat{\boldsymbol{A}}_{\tilde{p}, \tilde{p}}$ superimposed on the global spectrum of the cavity. Eight unstable global modes and 13 BPOD modes have been included in the reduced model. The global spectrum is coloured according to the criterion $\Gamma_{i}$.

$M_{3}$ in figure 10, rapidly converge towards stable global modes with a high value of the selection criterion $\Gamma_{i}$. We believe that this feature underlines the fact that these selected individual modes are rather important in describing the input-output behaviour. This stands as a validation of both the introduced selection criterion for global modes and the extraction of BPOD modes.

In particular, $M_{1}$ converges towards the 132th least stable global mode which has a rather high value of $\Gamma_{i}$ (see $E_{2}$ in table 1). This mode, displayed in figure 2(e), has an oscillatory frequency of $\omega=4.54$ and is responsible for capturing the peak in the transfer function (see figure $4 b$ ). The importance of this mode in terms of its contribution to the input-output relation is also visible in figure $5(b)$ where a significant drop in the error norm $\|\hat{H}-H\|_{\infty} /\|H\|_{\infty}$ occurs as soon as this particular global mode is included in the reduced-order model.

Note that $M_{1}, M_{2}$ and $M_{3}$ appear to belong to the same branch as the one comprising the unstable modes. This tendency should not come as a surprise as this branch is composed of shear-layer modes whose shape provides a link between the upstream edge of the cavity (where the actuator is located) and the downstream edge (where the sensor has been placed) through the shear-layer which acts as an amplifier. In a similar manner, the remaining modes in the BPOD spectrum account for more general structures that play an important role in the overall input-output relation.

\subsection{Reduced-order modelling using POD modes}

Coherent structures based on the POD are often used in deriving reduced-order models, and large body of literature exists which demonstrates their use in describing the essential dynamics of uncontrolled and controlled fluid systems by a set of equations with a significantly reduced number of degrees of freedom. For the sake of completeness, we will also consider POD modes as a basis for low dimensionally describing the input-output behaviour of our cavity problem. 


\subsubsection{Presentation of POD modes}

The classical snapshot method (see Sirovich 1987) is applied to compute POD modes for the stable subspace of our flow. This technique relies on the snapshots $\boldsymbol{T}$ collected during the impulse response simulation (see $\S 5.1$, identical to the dataset used for the BPOD computation).

The bi-orthogonal basis of the stable subspace is given by $\boldsymbol{V}_{s}=\boldsymbol{R}$ and $\boldsymbol{W}_{s}=\boldsymbol{S}$ with

$$
\boldsymbol{G}_{C} \boldsymbol{Q}_{1} \boldsymbol{R}_{1}=\boldsymbol{R}_{1} \boldsymbol{\Sigma}_{P O D}^{2}
$$

Since $\boldsymbol{G}_{C}=\boldsymbol{T}_{1} \boldsymbol{T}_{1}^{*}$, we obtain

$$
\begin{aligned}
\boldsymbol{T}_{1}^{*} \mathbf{Q}_{1} \boldsymbol{T}_{1} & =\boldsymbol{T}^{\prime} \boldsymbol{\Sigma}_{P O D}^{2} \boldsymbol{T}^{\prime *}, \\
\boldsymbol{T}^{\prime *} \boldsymbol{T}^{\prime} & =\boldsymbol{I}, \\
\boldsymbol{R} & =\boldsymbol{T} \boldsymbol{T}^{\prime} \boldsymbol{\Sigma}_{P O D}^{-1} .
\end{aligned}
$$

The adjoint POD modes are not equal to the direct POD modes due to the existence of an unstable subspace. The adjoint basis has to satisfy $\boldsymbol{S}^{*} \mathbf{Q} \boldsymbol{E}_{u}=0$ which leads to $\boldsymbol{S}=\left(\boldsymbol{P}_{s} \boldsymbol{R}\right)$.

For our configuration, i.e. flow over an open square cavity at $R e=7500$, the singular values $\boldsymbol{\Sigma}_{P O D}^{2}$ are depicted in figure 11(a). The plot shows the energy content of the coherent structures identified by the POD analysis, with a steady decay over four orders of magnitude for the first 50 POD modes. The corresponding modes are displayed, again by their streamwise velocity, in figure 11. Even though differences between these modes and the BPOD modes are clearly noticeable, the modes show finite amplitudes at the actuator and sensor location, thus anticipating their ability to represent the flow's input-output behaviour, even though this representation may not be accomplished as efficiently as by BPOD modes.

We would like to emphasize that these POD modes are not general; rather, they strongly depend on the chosen initial condition reflected in the control matrix $\boldsymbol{C}$. The extracted modes are optimal in representing the energetic structures triggered by the control. In other words, they can be regarded as the most controllable modes and, as a consequence, are an appropriate approximation basis for full-state information control. However, since no information about their measurement is taken into account in the construction of POD modes, we will show that BPOD-based models outperform POD-based models with respect to capturing the open-loop behaviour.

\subsubsection{Transfer function and error norm}

We then consider reduced-order models based on $p$ POD modes ranked by decreasing singular values. The transfer functions using a selected number of $p=10,28$ and 50 modes are displayed in figure 12(a). Similar to BPOD modes, the reduced-order model correctly approximates the exact transfer function provided that a sufficient number of POD modes are included. Contrary to BPOD modes, the number of modes required for a satisfactory approximation is substantially higher. Using 10 modes, the main peak is overestimated, and the transfer function displays large errors across the entire frequency spectrum. The transfer function of a model based on 28 POD modes shows oscillatory behaviour visible throughout the entire frequency domain, but the general behaviour is rather well predicted. As the number of modes is further increased, the approximate transfer functions match more closely the exact one.

The $\mathscr{H}_{\infty}$-error norm is displayed in figure $12(b)$. For very small-order models, the error increases, but, as more modes are added, the error decreases to reach values 
(b)
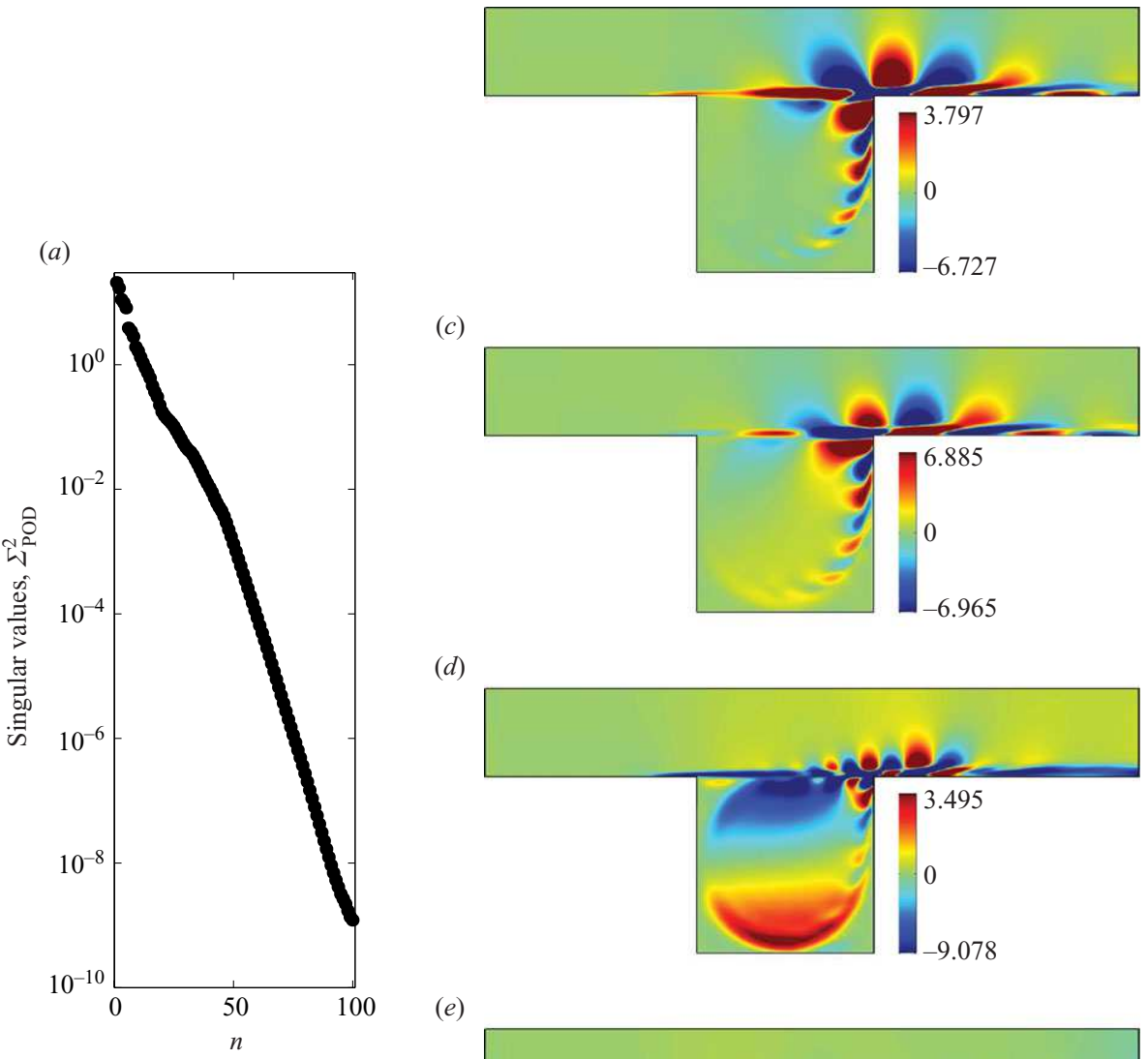

(c)

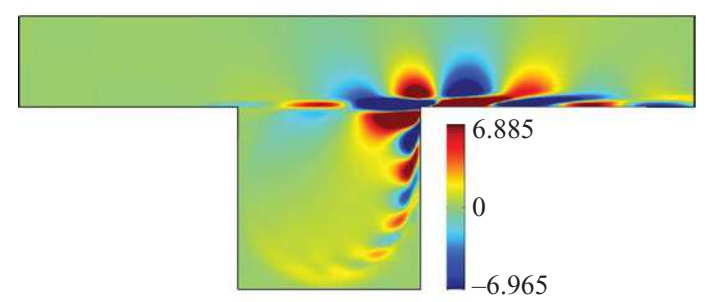

(d)

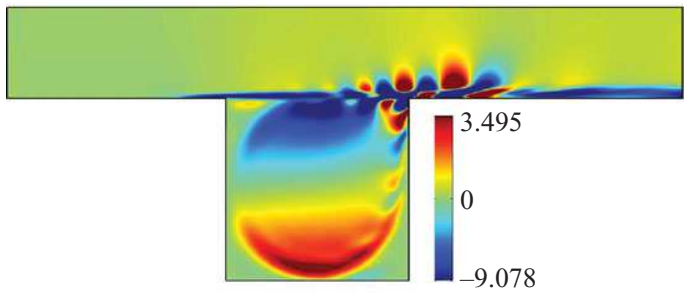

(e)

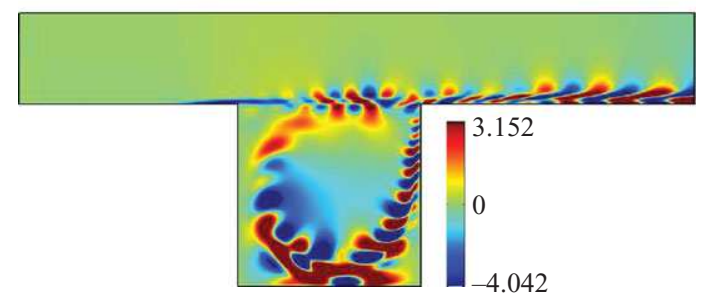

Figure 11. (a) Singular values for POD modes. $(b-e)$ POD modes for flow over an open square cavity at Reynolds number $R e=7500$. The $(b)$ first, $(c)$ second, $(d)$ third and $(e)$ twenty-eighth POD modes are visualized by their streamwise velocity component.

similar to those observed for using BPOD modes. We stress again that, for a given $\mathscr{H}_{\infty}$-error, the number of required POD modes is significantly higher than the number of required BPOD modes.

We observe that the reduced-order dynamics for the stable subspace spanned by the POD modes has always been stable no matter the number $p$ of POD modes. The same was not the case for the BPOD bases (see figure 9 where the reduced model based on nine BPOD modes was found unstable). Both POD- and BPOD-based reducedorder systems should be stable, independent of the number $p$ of included modes. In reality, however, the BPOD basis, though far more efficient, is also far more sensitive to numerical issues. POD modes are constructed using only information from the actuator whereas BPOD modes incorporate additional information from the 
(a)

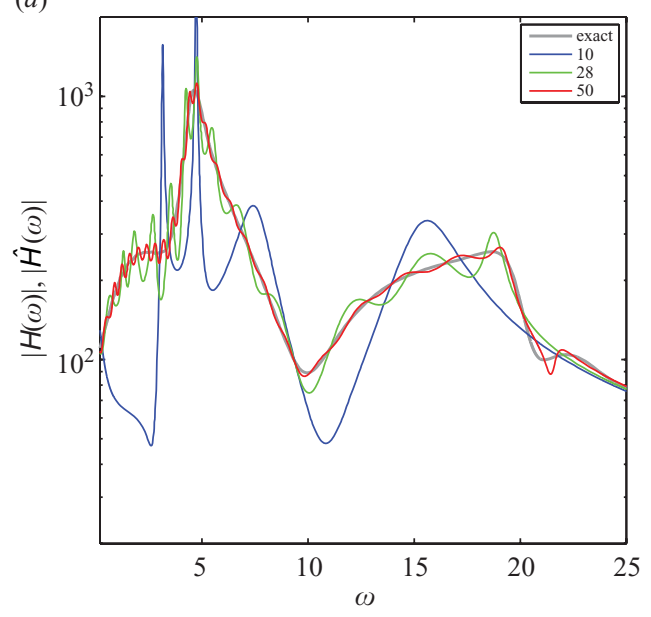

(b)

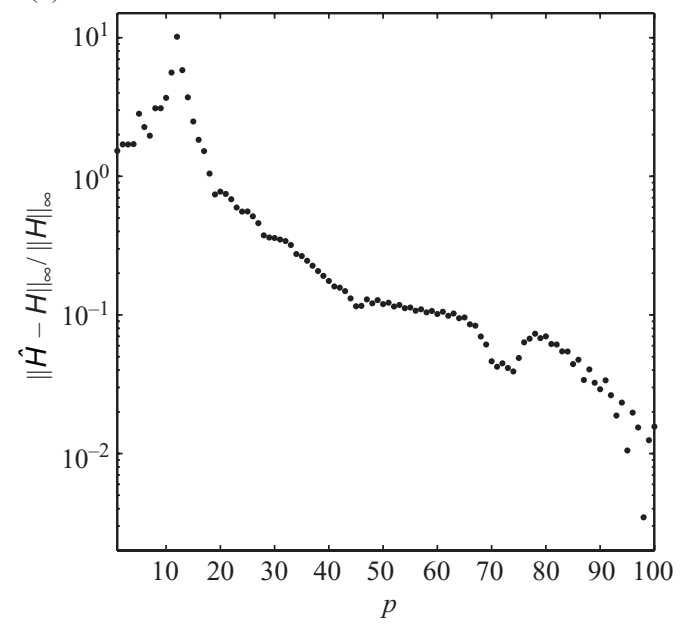

FiguRE 12. (a) Comparison of transfer function of the reduced-order model to the exact transfer function for a projection basis consisting of $p=10,28,50$ POD modes. ( $b$ ) Relative $\mathscr{H}_{\infty}$-error norm as a function of the number $p$ of included POD modes.

measurements. In the following section, we will present a comparison of BPOD and POD bases in terms of their observability.

\subsection{Comparison of BPOD and POD bases in terms of observability and controllability}

Controllability and observability have been identified as the critical quantities to measure the capability of a specific basis to accurately represent the input-output behaviour of a linear system. It seems reasonable then to analyse the POD and BPOD bases as to these two important criteria. To accomplish this we need to introduce a measure of observability for a given bi-orthogonal basis $(\boldsymbol{V}, \boldsymbol{W})$ that satisfies $\boldsymbol{W}^{*} \mathbf{Q} \boldsymbol{V}=\boldsymbol{I}$.

First, we recall that the direct BPOD basis, denoted by $\boldsymbol{J}_{1}$, is a solution of the following eigenvalue problem

$$
\mathbf{G}_{C} \mathbf{G}_{O} \boldsymbol{J}_{1}=\boldsymbol{J}_{1} \boldsymbol{\Sigma}^{2}
$$

where the controllability Gramian $\mathbf{G}_{C}$ can be decomposed according to $\mathbf{G}_{C}=\boldsymbol{T}_{1} \boldsymbol{T}_{1}^{*}$, and $\boldsymbol{T}_{1}$ represents a matrix whose columns contain the direct snapshots $\boldsymbol{X}_{1}(t)$ defined by (A 12). As shown by Rowley (2005), this result can be interpreted within a POD framework, since the direct BPOD basis coincides with the POD basis when the observability Gramian is used as a weight matrix in the scalar product, i.e. $\langle\boldsymbol{Y}, \boldsymbol{X}\rangle_{\mathbf{G}_{O}}=\boldsymbol{Y}^{*} \mathbf{G}_{O} \boldsymbol{X}$. This means that the first $i$ BPOD modes $\boldsymbol{J}_{1 i}$ form an orthogonal basis with respect to an observability-based scalar product and that this basis maximizes observability of the direct snapshots $\boldsymbol{X}_{1}(t)$. In addition, one may show that for the $i$ th BPOD structure $\boldsymbol{J}_{1 i}$, we obtain

$$
\int_{0}^{\infty}\left|\left\langle\boldsymbol{X}_{1}(t), \boldsymbol{J}_{1 i}\right\rangle_{\mathbf{G}_{O}}\right|^{2} \mathrm{~d} t=\boldsymbol{\Sigma}_{i}^{2}
$$

Hence, the square of the $i$ th Hankel singular value, $\Sigma_{i}^{2}$, is equivalent to the observability of the $i$ th BPOD mode, and consequently $\Sigma_{1}^{2}+\Sigma_{2}^{2}+\cdots+\Sigma_{i}^{2}$ is the observability of a basis consisting of the first $i$ BPOD structures.

We are now in a position to define the observability of a given bi-orthogonal basis $(\boldsymbol{V}, \boldsymbol{W})$. The snapshots $\boldsymbol{X}_{1}$ are first projected onto the subspace spanned by $\boldsymbol{V}$ using the 

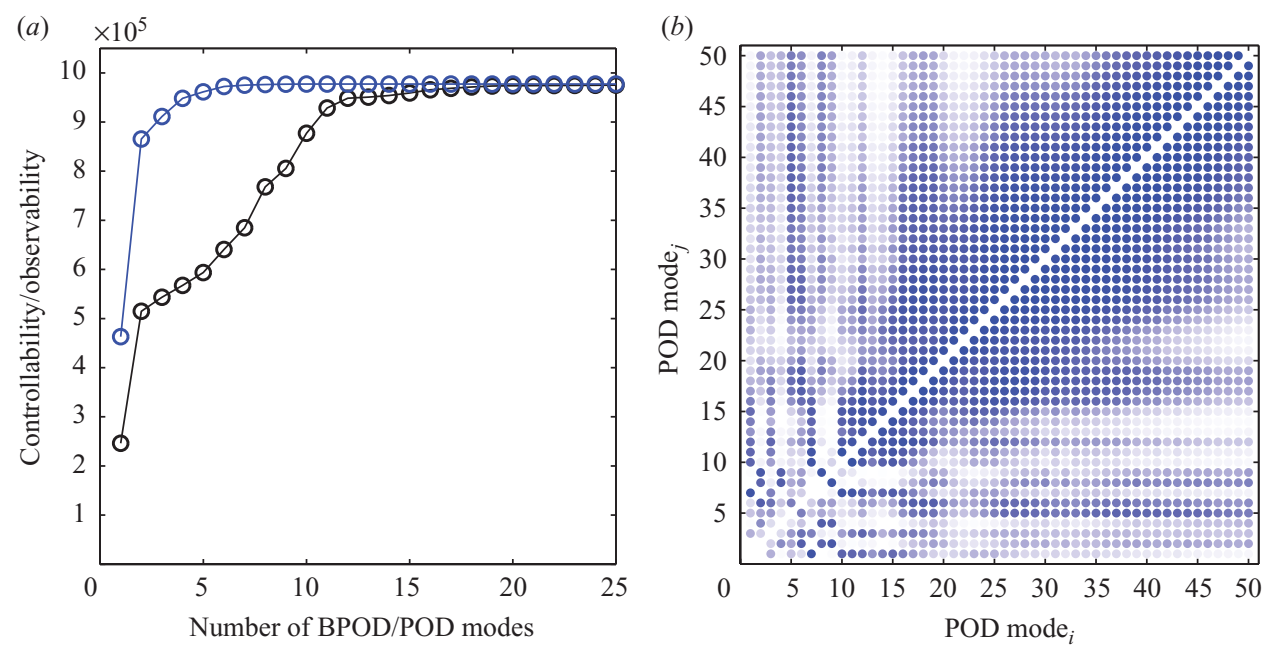

FIGURE 13. (a) Controllability/observability of a BPOD (blue) and POD (black) projection basis. (b) Observability measure of a POD basis according to criterion (5.27).

dual basis $\boldsymbol{W}$. This yields projected snapshots $\tilde{\boldsymbol{X}}_{1}$ and, hence, a projected controllability Gramian $\tilde{\boldsymbol{G}}_{C}=\tilde{\boldsymbol{T}}_{1} \tilde{\boldsymbol{T}}_{1}^{*}$. We proceed by using the true observability measure given by the observability Gramian $\boldsymbol{G}_{O}=\mathbf{Q}_{1} \boldsymbol{U}_{1} \boldsymbol{U}_{1}^{*} \boldsymbol{Q}_{1}$ and subsequently compute the eigenvalues of the large-scale system $\tilde{\boldsymbol{G}}_{C} \boldsymbol{G}_{O}$. For this, we perform a singular value decomposition of the expression $\boldsymbol{U}_{1}^{*} \boldsymbol{Q}_{1} \tilde{\boldsymbol{T}}_{1}$. The sum of the first $i$ eigenvalues $\tilde{\boldsymbol{\Sigma}}_{i}^{2}$ (the remaining eigenvalues are zero since the projection onto an $i$ dimensional basis results in a controllability Gramian $\tilde{\boldsymbol{G}}_{C}$ of rank $i$ ) quantifies the observability of the basis $(\boldsymbol{V}, \boldsymbol{W})$.

This procedure, applied to a BPOD basis of size $i$, yields the results shown in figure 13(a) by the blue symbols. This curve is simply the sum of the squared Hankel singular values of the original input-output system. We see that the observability of a basis consisting of $i$ BPOD modes quickly rises to reach a plateau for $i \geqslant 7$. For the bi-orthogonal POD bases introduced in $\S 5.4$, the results are displayed in the same figure by the black symbols. The observability of these POD-bases increases markedly slower and reaches the plateau, defined by the BPOD bases, only after a minimum of 20 POD modes are considered.

Incidentally, the controllability of a given bi-orthogonal basis $(\boldsymbol{V}, \boldsymbol{W})$ can be defined and analysed in an analogous way. In fact, the adjoint BPOD basis, denoted by $\boldsymbol{K}_{1}$, is the solution of the eigenvalue problem

$$
\mathbf{G}_{O} \boldsymbol{G}_{C}\left(\mathbf{Q}_{1} \boldsymbol{K}_{1}\right)=\left(\mathbf{Q}_{1} \boldsymbol{K}_{1}\right) \boldsymbol{\Sigma}^{2}
$$

where the observability Gramian $\boldsymbol{G}_{O}$ can be decomposed according to $\boldsymbol{G}_{O}=\boldsymbol{Q}_{1} \boldsymbol{U}_{1} \boldsymbol{U}_{1}^{*} \boldsymbol{Q}_{1}$, and $\boldsymbol{U}_{1}$ represents a matrix whose columns contain the adjoint snapshots $\boldsymbol{Y}_{1}(t)$ defined by (A 19). As before, an interpretation using a POD framework also holds in this case: the adjoint BPOD basis $\boldsymbol{Q}_{1} \boldsymbol{K}_{1}$ corresponds to the POD basis associated with the adjoint snapshots $\boldsymbol{Y}_{1}(t)$ and a scalar product based on the controllability Gramian, i.e. $\langle\boldsymbol{Y}, \boldsymbol{X}\rangle_{\mathbf{G}_{C}}=\boldsymbol{Y}^{*} \boldsymbol{G}_{C} \boldsymbol{X}$. The adjoint basis is thus orthogonal with respect to a controllability-based scalar product and maximizes controllability of the adjoint snapshots $\boldsymbol{Y}_{1}(t)$.

We then propose to define the controllability of a given bi-orthogonal basis as follows. The adjoint snapshots $\boldsymbol{Y}_{1}$ are projected onto the subspace spanned by the dual 
basis $\boldsymbol{W}$ using the primal basis $\boldsymbol{V}$. This yields projected snapshots $\tilde{\boldsymbol{Y}}_{1}$ and a projected observability Gramian $\tilde{\boldsymbol{G}}_{O}=\boldsymbol{Q}_{1} \tilde{\boldsymbol{U}}_{1} \tilde{\boldsymbol{U}}_{1}^{*} \mathbf{Q}_{1}$. Using the true controllability measure given by the controllability Gramian $\boldsymbol{G}_{C}=\boldsymbol{T}_{1} \boldsymbol{T}_{1}^{*}$, we compute the eigenvalues of the largescale system $\tilde{\boldsymbol{G}}_{O} \boldsymbol{G}_{C}$. In other words, we perform a singular value decomposition of the expression $\tilde{\boldsymbol{U}}_{1}^{*} \boldsymbol{Q}_{1} \boldsymbol{T}_{1}$. The sum of the first $i$ eigenvalues $\tilde{\boldsymbol{\Sigma}}_{i}^{2}$ constitutes the controllability of the basis. The results are given in figure 13, showing values identical to those obtained for the observability analysis.

An alternative way to compare the POD and the BPOD basis is to evaluate the non-orthogonality of the POD basis with respect to the observability based scalar product. We recall that the POD basis is orthogonal with respect to the energybased scalar product while the direct BPOD basis is orthogonal with respect to the observability based scalar product. To compare the two measures, i.e. the energy and the observability measures, we may, for example, evaluate directly the nonorthogonality of the POD basis with respect to the scalar product based on the observability Gramian. Hence, we compute for two POD modes, $\boldsymbol{R}_{1 i}$ and $\boldsymbol{R}_{1 j}$, the quantity

$$
\left|\frac{\boldsymbol{R}_{1 i}^{*} \boldsymbol{G}_{O} \boldsymbol{R}_{1 j}}{\sqrt{\boldsymbol{R}_{1 i}^{*} \mathbf{G}_{O} \boldsymbol{R}_{1 i}} \sqrt{\boldsymbol{R}_{1 j}^{*} \boldsymbol{G}_{O} \boldsymbol{R}_{1 j}}}-\delta_{i j}\right|,
$$

which would be zero if the energy measure based on $\boldsymbol{Q}_{1}$ were equal to the observability measure based on $\boldsymbol{G}_{O}$. Each off-diagonal coefficient of this matrix lies within the interval $[0,1]$, and the diagonal coefficients are identically zero by construction. If an off-diagonal term is close to zero, the two corresponding POD modes are nearly orthogonal with respect to the observability-based scalar product (in addition to being orthogonal with respect to the energy-based inner product). If, on the other hand, an off-diagonal term is close to one, the two corresponding POD modes are nearly collinear with respect to the observability criterion, even though they are orthogonal with respect to the energy-based inner product.

In figure $13(b)$ we visualize the matrix defined above for the first 50 POD modes. We observe significant off-diagonal coefficients (indicated by dark blue symbols representing values close to one) which further supports our previous findings and provides additional evidence that POD bases are inferior to BPOD bases when the full-scale input-output behaviour of a fluid system has to be approximated in a lower-dimensional yet efficient manner.

\section{Closed-loop control}

Among the various bases that have been considered in the previous section, global modes were unsuitable to represent the input-output behaviour while BPOD modes and (to a lesser degree) POD modes were capable of reproducing the transfer function of the full system. In this section we further expound on these findings by considering the complete problem of designing feedback control strategies to stabilize the globally unstable cavity flow. This step constitutes an important step in the performance analysis of reduced-order controllers, since the $\mathscr{H}_{\infty}$-error in approximating the exact transfer function gives a first indication of the suitability and effectiveness of the chosen expansion basis but does not allow definite conclusions about the performance of the closed-loop system.

The linearized Navier-Stokes equations (defined in (2.8)) represent the plant to be controlled. For the computation of the control law and the design of the estimator, 
a reduced-order model based on (4.3) and (4.7) will be used. This model consists of an unstable subspace which is represented by the unstable global modes and a stable subspace which is modelled by either global modes, BPOD modes or POD modes. After a brief explanation of the LQG framework, the performance of the closed-loop control applied to the linearized numerical simulation will be studied for each of the above bases. In this effort the stability of the compensated system will emerge as a relevant quantity and will be linked to the open-loop behaviour of the model reduction bases.

\subsection{The $L Q G$ framework}

A description that is particularly suited for many fluid dynamical application is based on the LQG framework (Zhou et al. 2002). In this formulation, one describes the controlled system by a linear (L) model, a quadratic $(\mathrm{Q})$ cost functional, and an external Gaussian $(\mathrm{G})$ stochastic noise source.

The design of an LQG system consists of two principal steps: the design of a full-state information controller, and the design of an estimator. The estimator's role is to reconstruct, in the best possible manner, the reduced state vector $\hat{\boldsymbol{X}}$ based only on the discrepancy of the measurements from the plant and the estimator. The role of the controller is the forcing of the linear system such that a specified cost objective is reached. The input for this control stems from the estimator. The above-mentioned two principal steps can be taken independent from each other due to the well-known separation principle of control theory (Zhou et al. 2002; Kim \& Bewley 2007).

We start by designing the full-state information controller and assume a linear relation between the reduced state vector $\hat{\boldsymbol{X}}$ and the control $c$,

$$
c=\hat{\boldsymbol{K}} \hat{\boldsymbol{X}} \text {. }
$$

The control gain $\hat{\boldsymbol{K}}$ can be determined from a variational principle that minimizes the cost functional $\hat{\boldsymbol{X}}^{*} \hat{\boldsymbol{T}} \hat{\boldsymbol{X}}+\ell^{2} c^{2}$ subject to the constraint of $\hat{\boldsymbol{X}}$ and $c$ satisfying the governing equations. The user-specified parameter $\ell^{2}$ determines the cost of the control effort. $\hat{\boldsymbol{T}}$ is a Hermitian matrix describing the cost functional. Usually $\hat{\boldsymbol{T}}$ represents the kinetic disturbance energy according to $\hat{\boldsymbol{T}}=\boldsymbol{V}^{*} \boldsymbol{Q} \boldsymbol{V}$ but it may also be chosen as the square of the measurement signal $\hat{\boldsymbol{T}}=\boldsymbol{V}^{*} \boldsymbol{M}^{*} \boldsymbol{M} \boldsymbol{V}$.

The resulting optimal control gain $\hat{\boldsymbol{K}}$ can be expressed as $\hat{\boldsymbol{K}}=-\hat{\mathbf{C}} \hat{\boldsymbol{K}}^{\prime}$ where $\hat{\boldsymbol{K}}^{\prime}$ satisfies the algebraic matrix Riccati equation

$$
\hat{\boldsymbol{A}}^{*} \hat{\boldsymbol{K}}^{\prime}+\hat{\boldsymbol{K}}^{\prime} \hat{\boldsymbol{A}}-\hat{\boldsymbol{K}}^{\prime} \hat{\boldsymbol{C}} \hat{\boldsymbol{C}}^{*} \hat{\boldsymbol{K}}^{\prime}+\frac{\hat{\boldsymbol{T}}}{\ell^{2}}=0
$$

With the relation between $c$ and $\hat{\boldsymbol{X}}$ established we can close the loop on the reduced model and formulate the reduced-order closed-loop system as

$$
\frac{\mathrm{d} \hat{\boldsymbol{X}}}{\mathrm{d} t}=(\hat{\boldsymbol{A}}+\hat{\boldsymbol{C}} \hat{\boldsymbol{K}}) \hat{\boldsymbol{X}}
$$

where $\hat{\boldsymbol{A}}+\hat{\boldsymbol{C}} \hat{\boldsymbol{K}}$ is a stable evolution operator contrary to $\hat{\boldsymbol{A}}$ which is unstable.

The second step, the design of the estimator, commences with the formulation of the estimator system which will govern the approximation $\hat{\boldsymbol{Y}}$ to the exact reduced state 
vector $\hat{\boldsymbol{X}}$. We have

$$
\frac{\mathrm{d} \hat{\boldsymbol{Y}}}{\mathrm{d} t}=\hat{\boldsymbol{A}} \hat{\boldsymbol{Y}}+\hat{\mathbf{C}} c-\hat{\boldsymbol{L}}(m-\hat{\boldsymbol{M}} \hat{\boldsymbol{Y}}) .
$$

where $c=\hat{\boldsymbol{K}} \hat{\boldsymbol{Y}}$ implements the previously defined control term. An additional control term of the form $\hat{\boldsymbol{L}}(m-\hat{\boldsymbol{M}} \hat{\boldsymbol{Y}})$ appears which exerts a forcing on the estimator given by the difference between the measurement $m$ and the measurement $\hat{M} \hat{Y}$ of the estimated system. The matrix $\hat{L}$, referred to as the Kalman gain, determines the manner in which this measurement difference is applied.

Analogous to the control gain $\hat{\boldsymbol{K}}$, the Kalman gain $\hat{\boldsymbol{L}}$ can be computed by invoking a variational problem which aims at minimizing the state estimation error while observing the governing equations (6.4). The Kalman gain is then given as $\hat{\boldsymbol{L}}=-\hat{\boldsymbol{L}}^{\prime} \hat{\boldsymbol{M}}^{*}$ where $\hat{L}^{\prime}$ satisfies the algebraic Riccati equation

$$
\hat{\boldsymbol{A}} \hat{L}^{\prime}+\hat{\boldsymbol{L}}^{\prime} \hat{\boldsymbol{A}}^{*}-\hat{\boldsymbol{L}}^{\prime} \hat{\boldsymbol{M}}^{*} \hat{\mathbf{M}} \hat{\boldsymbol{L}}^{\prime}+\frac{W^{2}}{G^{2}} \hat{\mathbf{N}} \hat{\boldsymbol{N}}^{*}=0 .
$$

Here, $\hat{\boldsymbol{N}}$ and $W^{2}$ characterize, respectively, the correlation and the variance of the plant noise while $G^{2}$ denotes the variance of the measurement noise.

In a final step, the controlled system and the estimator are combined to form a compensated system. This system is given by the set of equations

$$
\begin{aligned}
& \frac{\mathrm{d}}{\mathrm{d} t}\left(\begin{array}{l}
\hat{\boldsymbol{X}} \\
\hat{\boldsymbol{Y}}
\end{array}\right)=\left(\begin{array}{cc}
\hat{\boldsymbol{A}} & \hat{\boldsymbol{C}} \hat{\boldsymbol{K}} \\
-\hat{\boldsymbol{L}} \hat{\boldsymbol{M}} & \hat{\boldsymbol{A}}+\hat{\boldsymbol{C}} \hat{\boldsymbol{K}}+\hat{\boldsymbol{L}} \hat{\boldsymbol{M}}
\end{array}\right)\left(\begin{array}{l}
\hat{\boldsymbol{X}} \\
\hat{\boldsymbol{Y}}
\end{array}\right), \\
&\left.\left(\begin{array}{c}
\hat{\boldsymbol{X}} \\
\hat{\boldsymbol{Y}}
\end{array}\right)\right|_{t=0}=\left(\begin{array}{c}
\boldsymbol{W}^{*} \boldsymbol{Q} \boldsymbol{X}(0) \\
0
\end{array}\right) .
\end{aligned}
$$

We would like to point out that the full-state information controller $\hat{\boldsymbol{C}} \hat{\boldsymbol{K}} \hat{\boldsymbol{X}}$ in (6.3) has been replaced by the controller $\hat{\boldsymbol{C}} \hat{\boldsymbol{K}} \hat{\boldsymbol{Y}}$ using the estimated rather than the exact reduced state vector. In the above system, only the measurements of the full system are used, making this type of control design more promising for a realistic implementation.

An equally convenient and instructive formulation consists of replacing the estimated reduced state vector $\hat{\boldsymbol{Y}}$ by the state estimation error $\hat{\boldsymbol{Z}}=\hat{\boldsymbol{X}}-\hat{\boldsymbol{Y}}$ (Kim \& Bewley 2007). A straightforward manipulation results in

$$
\begin{aligned}
\frac{\mathrm{d}}{\mathrm{d} t}\left(\begin{array}{l}
\hat{\boldsymbol{X}} \\
\hat{\boldsymbol{Z}}
\end{array}\right) & =\left(\begin{array}{cc}
\hat{\boldsymbol{A}}+\hat{\boldsymbol{C}} \hat{\boldsymbol{K}} & -\hat{\boldsymbol{C}} \hat{\boldsymbol{K}} \\
0 & \hat{\boldsymbol{A}}+\hat{\boldsymbol{L}} \hat{\boldsymbol{M}}
\end{array}\right)\left(\begin{array}{c}
\hat{\boldsymbol{X}} \\
\hat{\boldsymbol{Z}}
\end{array}\right), \\
\left.\left(\begin{array}{c}
\hat{\boldsymbol{X}} \\
\hat{\boldsymbol{Z}}
\end{array}\right)\right|_{t=0} & =\left(\begin{array}{c}
\boldsymbol{W}^{*} \boldsymbol{Q} \boldsymbol{X}(0) \\
\boldsymbol{W}^{*} \boldsymbol{Q} \boldsymbol{X}(0)
\end{array}\right),
\end{aligned}
$$

which demonstrates that due to the block-triangular shape of the composite system matrix in (6.7a), the controller design and estimator design decouple, illustrating the separation principle of control theory. For this reason, the control $\hat{\boldsymbol{\kappa}}$, determined under the assumption of full-state information, does not have to be recomputed when used with an estimated rather than an exact reduced state vector. The LQG framework insures that $\hat{\boldsymbol{A}}+\hat{\boldsymbol{C}} \hat{\boldsymbol{K}}$ and $\hat{\boldsymbol{A}}+\hat{\boldsymbol{L}} \hat{\boldsymbol{M}}$ are stable while the reduced system $\hat{\boldsymbol{A}}$ is unstable (Burl 1999). 
Note that we use an augmented system in this study where the flow-state includes both the reduced dynamics $\hat{\boldsymbol{X}}$ and the magnitude of the blowing/suction $\rho(t)$ :

$$
\begin{aligned}
\frac{\mathrm{d}}{\mathrm{d} t}\left(\begin{array}{c}
\hat{\boldsymbol{X}} \\
\rho
\end{array}\right) & =\left(\begin{array}{ll}
\hat{\boldsymbol{A}} & 0 \\
0 & 0
\end{array}\right)\left(\begin{array}{c}
\hat{\boldsymbol{X}} \\
\rho
\end{array}\right)+\left(\begin{array}{c}
\hat{\boldsymbol{C}} \\
-1
\end{array}\right) c, \\
m & =\left(\begin{array}{ll}
\hat{\boldsymbol{M}} & 0
\end{array}\right)\left(\begin{array}{c}
\hat{\boldsymbol{X}} \\
\rho
\end{array}\right) .
\end{aligned}
$$

This step enables us to penalize both the magnitude $\rho(t)$ of blowing/suction and its derivative $c(t)=-\mathrm{d} \rho / \mathrm{d} t$. Hence, it allows the control effort - and not only its first time-derivative - to be zero at infinite time. For the sake of simplicity, the derivations to follow will tacitly assume this arrangement.

\subsection{Small-gain limit}

An interesting limit arises in the above equations as the cost of control and the ratio between measurement noise and plant noise tends to infinity, i.e. $\ell^{2} \rightarrow \infty$ and $G^{2} / W^{2} \rightarrow \infty$. In this case, any exerted control and estimation effort concentrates on suppressing and estimating the unstable modes of the uncontrolled system.

In this small-gain limit the control and Kalman gains take on the respective special form (see Burl 1999):

$$
\begin{array}{ll}
\hat{\boldsymbol{K}}=\left(\begin{array}{ll}
\hat{\boldsymbol{K}}_{u} & 0
\end{array}\right), & \hat{\boldsymbol{K}}_{u}=-\hat{\boldsymbol{C}}_{u} \hat{\boldsymbol{K}}_{u}^{\prime}, \\
\hat{\boldsymbol{L}}=\left(\begin{array}{c}
\hat{\boldsymbol{L}}_{u} \\
0
\end{array}\right), \quad \hat{\boldsymbol{L}}_{u}=-\hat{\boldsymbol{L}}_{u}^{\prime} \hat{\boldsymbol{M}}_{u}^{*}
\end{array}
$$

with

$$
\begin{aligned}
\hat{\boldsymbol{\Lambda}}_{u}^{*} \hat{\boldsymbol{K}}_{u}^{\prime}+\hat{\boldsymbol{K}}_{u}^{\prime} \hat{\boldsymbol{\Lambda}}_{u}-\hat{\boldsymbol{K}}_{u}^{\prime} \hat{\boldsymbol{C}}_{u} \hat{\boldsymbol{C}}_{u}^{*} \hat{\boldsymbol{K}}_{u}^{\prime}=0, \\
\hat{\boldsymbol{\Lambda}}_{u} \hat{\boldsymbol{L}}_{u}^{\prime}+\hat{\boldsymbol{L}}_{u}^{\prime} \hat{\boldsymbol{\Lambda}}_{u}^{*}-\hat{\boldsymbol{L}}_{u}^{\prime} \hat{\boldsymbol{M}}_{u}^{*} \hat{\boldsymbol{M}}_{u} \hat{\boldsymbol{L}}_{u}^{\prime}=0 .
\end{aligned}
$$

Hence the control and Kalman gains reduce in the small gain limit to the gains pertaining only to the unstable modes. The Riccati equations to be solved are therefore of very low dimension. Note that an analytical formulation for the gains in the small-gain limit has been derived in Lauga \& Bewley (2003). In addition, it can be shown that the unstable eigenvalues of the uncontrolled system are reflected about the imaginary axis (see Burl 1999) when control is applied. In this case the matrix $\hat{\boldsymbol{T}}$ related to the cost functional and the matrix $\hat{\boldsymbol{N}}$ characterizing the plant noise are no longer significant here and, therefore, do not need to be defined.

To insure small amplitudes in the gains $\hat{\boldsymbol{K}}$, the location of the actuator is chosen such that $\hat{\mathbf{C}}=\boldsymbol{W}^{*} \mathbf{Q C}$ is sufficiently large for the unstable global modes. Higher values of $\boldsymbol{W}^{*} \mathbf{Q C}$ will be attained if the actuator location coincides with regions of high amplitudes of the adjoint modes. The adjoint modes thus indicate the location where the corresponding global modes can be easily excited. This actuator placement procedure ensures reasonable controllability of the unstable global modes, a prerequisite for an effective control design. The values of $\hat{\boldsymbol{C}}_{i}=\boldsymbol{W}_{i}^{*} \mathbf{Q C}$ for the global modes $E_{-3}, E_{-2}, \ldots, E_{8}$ are given in table 1. Also, to ensure small amplitudes in the Kalman gain $\hat{\boldsymbol{L}}$, the sensor location is chosen such that $\hat{\boldsymbol{M}}=\boldsymbol{M V}$ is sufficiently large for the unstable global modes. Under this condition, the unstable global modes are reasonably observable. The values of $\hat{\boldsymbol{M}}_{i}=\boldsymbol{M E}_{i}$ for the unstable global modes are given in table 1. 

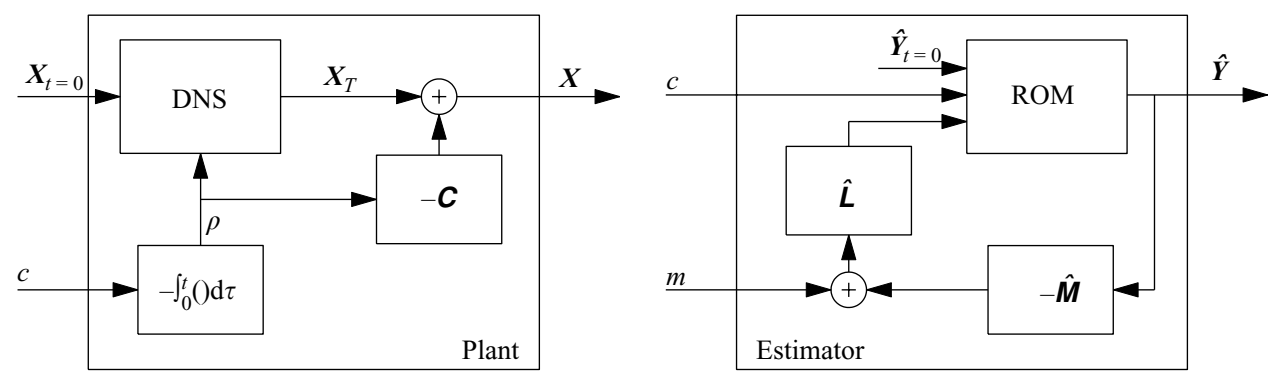

FIGURE 14. Block diagrams for the DNS module (plant) based on (2.8) and the estimator module based on a reduced-order model (ROM) described by (6.4).

The special form of the control and Kalman gain allows the partitioning of the compensated system in matrix form into stable (subscript ${ }_{s}$ ) and unstable (subscript ${ }_{u}$ ) components as follows:

$$
\frac{\mathrm{d}}{\mathrm{d} t}\left(\begin{array}{c}
\hat{\boldsymbol{X}}_{s} \\
\hat{\boldsymbol{X}}_{u} \\
\hat{\boldsymbol{Z}}_{u} \\
\hat{\boldsymbol{Z}}_{s}
\end{array}\right)=\left(\begin{array}{ccc|c}
\hat{\boldsymbol{A}}_{s} & \hat{\boldsymbol{C}}_{s} \hat{\boldsymbol{K}}_{u} & -\hat{\boldsymbol{C}}_{s} \hat{\boldsymbol{K}}_{u} & 0 \\
0 & \hat{\boldsymbol{A}}_{u}+\hat{\boldsymbol{C}}_{u} \hat{\boldsymbol{K}}_{u} & -\hat{\boldsymbol{C}}_{u} \hat{\boldsymbol{K}}_{u} & 0 \\
0 & 0 & \hat{\boldsymbol{A}}_{u}+\hat{\boldsymbol{L}}_{u} \hat{\boldsymbol{M}}_{u} & \hat{\boldsymbol{L}}_{u} \hat{\boldsymbol{M}}_{s} \\
\hline 0 & 0 & 0 & \hat{\boldsymbol{A}}_{s}
\end{array}\right)\left(\begin{array}{c}
\hat{\boldsymbol{X}}_{s} \\
\hat{\boldsymbol{X}}_{u} \\
\hat{\boldsymbol{Z}}_{u} \\
\hat{\boldsymbol{Z}}_{s}
\end{array}\right) .
$$

Thus, provided the reduced models for the plant $\hat{\boldsymbol{X}}$ and for the estimator $\hat{\boldsymbol{Y}}$ are the same, the eigenvalues of the compensated system are those of $\hat{\boldsymbol{A}}_{s}, \hat{\boldsymbol{A}}_{u}+\hat{\boldsymbol{C}}_{u} \hat{\boldsymbol{K}}_{u}$ and $\hat{\boldsymbol{A}}_{u}+\hat{\boldsymbol{L}}_{u} \hat{\boldsymbol{M}}_{u}$. The performance, usually assimilated as the least stable eigenvalue (long-term smallest decay rate), can then be predicted theoretically.

If the plant $\hat{\boldsymbol{X}}$ is based on the complete set of global modes, the equation acting on $\hat{\boldsymbol{X}}$ in (6.11) is equivalent to the full DNS. If, in addition, the estimated state $\hat{\boldsymbol{Y}}$ is also based on the complete set of global modes, we obtain the 'best control strategy' which will be presented in $\S 6.3 .3$. In this case, we know that in the small-gain limit the spectrum of this compensated system is composed of the stable modes of the uncontrolled simulation (coloured eigenvalues in figure 10) and the reflection of the unstable modes into the stable half-plane. The small-gain limit will be invoked in what follows.

\subsection{Analysis of the performance of the compensated system using DNS}

For clarity and throughout this investigation, we will present the various control configurations in block-diagram form which will help the reader to evaluate and appreciate the many options and approximations in the design and operation of control strategies. Two main blocks, the plant and the estimator, are principal components of any subsequent control scheme. They are displayed in figure 14 . The plant module takes as input the initial condition and the control $c$ and produces a time sequence of state vectors $\boldsymbol{X}$. Internally, this is accomplished by integrating the control variable $c$ to obtain $\rho$ and by subtracting the solution $\boldsymbol{C}$ of the inhomogeneous problem (with unit forcing at the control location). The internal structure of the DNS module implements the lifting procedure. The estimator module has the task of providing an approximate state vector based on the external measurements $m$ and the internally generated measurements. The Kalman gain $\hat{L}$ is given by $(6.9 b)$ and $(6.10 b)$. The estimator is driven by a control law $c$ which is the same as the control law that controls the plant. 
(a)

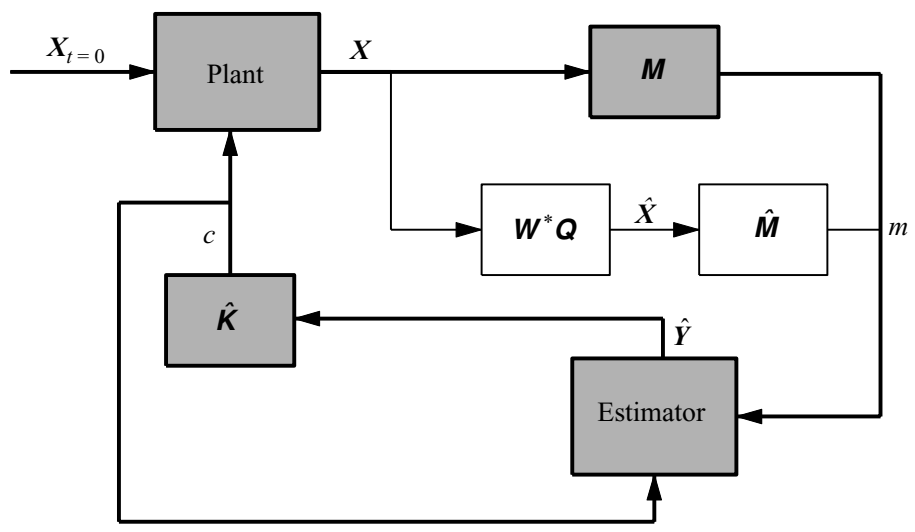

(b)

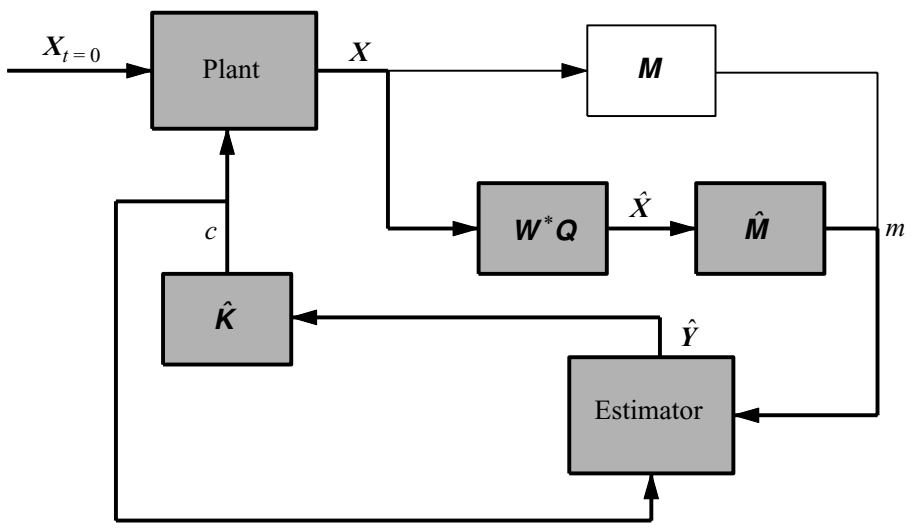

FIGURE 15. Block diagrams demonstrating $(a)$ partial-state information control and $(b)$ 'best control strategy'. Only the shaded blocks and the thick lines are active in each diagram. The details of the plant and estimator blocks are given in figure 14.

We now turn our attention to the case of control with partial-state information. A schematic of this set-up in form of a block diagram is given in figure 15(a). Once the estimator and controller are defined, the procedure for partial-state information control progresses as follows. The DNS of the flow over a cavity produces a flow field $\boldsymbol{X}(t)$. The sensor, located at the downstream edge of the cavity, extracts from this flow field a skin friction signal via $m(t)=\boldsymbol{M} \boldsymbol{X}(t)$, which constitutes the only flow information available to the compensator. The signal $m(t)$ is subsequently fed into the estimator which in turn yields an estimate $\hat{\boldsymbol{Y}}(t)$ of the flow field. In a final step, the controller converts the estimated flow field $\hat{\boldsymbol{Y}}(t)$ into a control law $c(t)$ according to $c(t)=\hat{\boldsymbol{K}} \hat{\boldsymbol{Y}}(t)$ which governs the blowing/suction strength at the upstream edge of the cavity in the numerical simulation, thus completing the feedback cycle. $\hat{\boldsymbol{K}}$ is given by $(6.9 a)$ and $(6.10 a)$.

\subsubsection{Performance of a reduced-order model based on BPOD modes}

We start by assessing the performance of reduced-order model-based estimators where the eight unstable global modes and a series of $p$ BPOD modes are taken into account. In figure 16(a) we plot the energy $\boldsymbol{X}^{*} \boldsymbol{Q} \boldsymbol{X}$ as a function of time for various partial-state control simulations. Each curve represents a different number $p$ of BPOD modes included in the reduced-order model. The uppermost thin line 

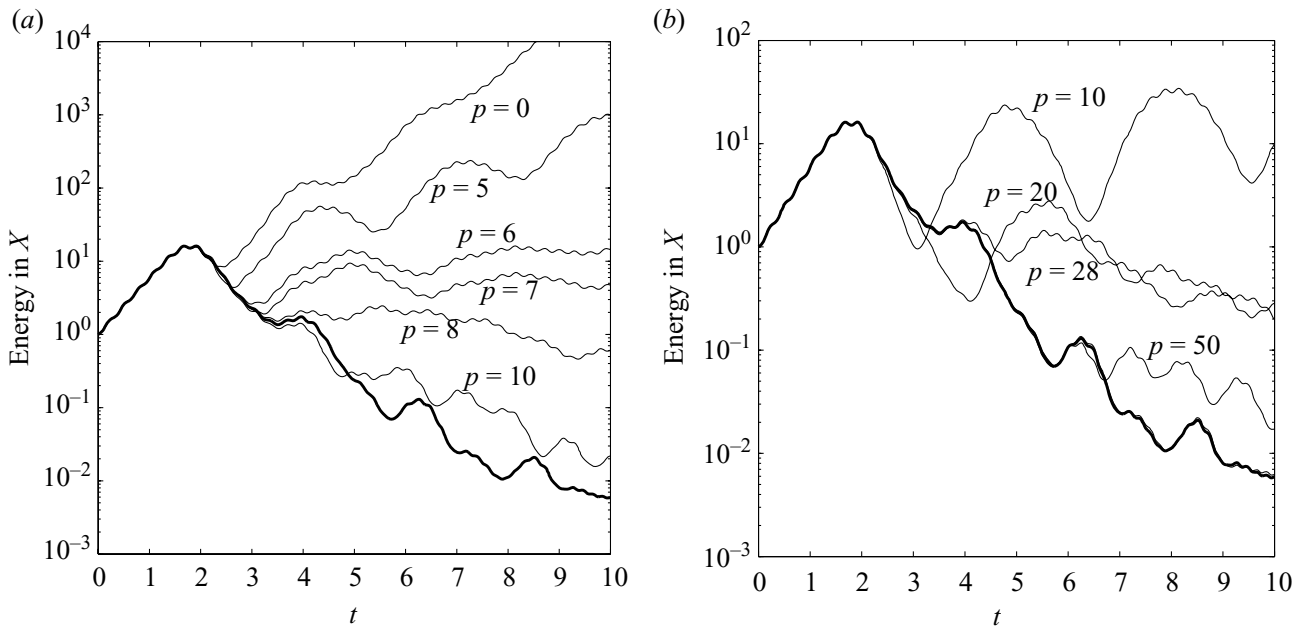

FIGURE 16. Performance of partial-state controller measured as the perturbation kinetic energy versus time for different reduced-order models. $(a)$ The number of included BPOD modes (in addition to the unstable global modes) is denoted by $p$. (b) The number of included POD modes (in addition to the unstable global modes) is denoted by $p$.

corresponds to an estimator based on a reduced-order model with the eight unstable global modes and no BPOD modes $(p=0)$.

The energy diverges which indicates that this specific reduced-order model (based on unstable global modes only) is not able to provide an accurate estimate of the flow field. Thus, in order to suppress a global instability, modelling only the unstable subspace is not sufficient; rather, a representation of the stable subspace has to be included in the reduced-order model. In view of the results on the reduction of the stable subspace (see $\S 5$ ), it may come as a surprise that using a few stable modes (for instance only two stable modes are considered in Åkervik et al. 2007) will provide sufficient information about the stable subspace to result in a successful control effort. A similar divergent behaviour can be observed for the two next-lower thin lines which correspond to reduced-order models based on five and six BPOD modes, respectively. The divergence though is less severe which we take as an indication that the performance of the estimator improves as the number of included BPOD modes increases. A breakeven point is reached for $p=7$, when the partial-state control manages to keep the energy bounded; for $p=8$ the energy starts to decrease. The sequence of curves depicted in figure $16(a)$ and labelled by $p=0,5,6, \ldots$ converge towards a curve (thick solid line) which corresponds to the best control achievable using partial-state control (see $\S 6.3 .3$ ).

\subsubsection{Performance of a reduced-order model based on POD modes}

We continue our evaluation of reduction bases by studying reduced-order models based on the eight unstable global modes and a series of $p$ POD modes. The kinetic energy of the perturbations is displayed in figure 16(b). A similar behaviour as for the BPOD modes is recovered: when only a few modes are considered in the reducedorder model, the flow cannot be successfully stabilized whereas the energy behaviour converges to the 'best control strategy' curve (thick solid line) when more POD modes are added. This behaviour corroborates the results obtained from our analysis of the open-loop response. We however emphasize the fact that the order of the stable 
reduced model using POD modes is higher than the one based on BPOD modes which is again in complete agreement with the results of our open-loop analysis.

\subsubsection{Convergence towards the 'best control strategy'}

Using reduced-order models based on BPOD and POD modes, we have seen that while the order of the model is increased, the input-output behaviour is better captured and the energy curves converge to a particular curve, the 'best control strategy'. In this section, we will show that this optimum solution may be obtained through a full-state control strategy, which only involves the unstable global modes in the reduced-order model.

We first emphasize the fact that the estimators and the controllers are based on a reduced model. In particular, we recall the equation governing the approximation of the reduced state (defined in (6.4))

$$
\frac{\mathrm{d} \hat{\boldsymbol{Y}}}{\mathrm{d} t}=\hat{\boldsymbol{A}} \hat{\boldsymbol{Y}}+\hat{\boldsymbol{C}}_{c}-\hat{\boldsymbol{L}}(m-\hat{\boldsymbol{M}} \hat{\boldsymbol{Y}})
$$

in which the quantity $(m-\hat{\mathbf{M}} \hat{\boldsymbol{Y}})$ is minimized. It is then clear that the relevant quantity to be fed into the estimator is not $m$ which corresponds to the measurement of the whole flow, but a reduced measure $\hat{m}$ which corresponds to the measurement arising from the modes comprised in the reduced model, i.e. $\hat{m}=\hat{\boldsymbol{M}} \hat{\boldsymbol{X}}$. This is done in the 'best control strategy', illustrated in form of a block diagram in figure 15(b). At first sight it seems similar to the above partial-state information control set-up; but closer inspection shows that the measurement signal to be provided to the estimator is the projected measurement $\hat{m}(t)$ rather than the true measurement $m(t)$. Note that this control strategy requires knowledge of the full flow field and is thus closer to a full-state than to a partial-state information control strategy. It is only considered here as an analysis tool. It is important to note that within the best control strategy the same result (same control law, same simulation) is obtained regardless of the number $p$ of included vectors in the stable subspace. In particular, the best control strategy may be determined with $p=0$, i.e. with only eight unstable global modes in the reduced-order model of the estimator.

Applying this control strategy, we obtain the thick black lines in figures 16(a) and 16(b). The partial-state simulations in these figures therefore converge towards this curve as the performance of reduced-order models is increased. The energy first increases exponentially up to $t \approx 2$, after which we observe a drastic decrease. More insight into the control's physics can be gained by looking at the evolution of the pressure field taken along the line $y=0$. This is shown in the form of an $(x, t)$ plane in figure 17 . We notice that the pressure signal first increases, reaching its maximum at time $t \approx 2$. During this initial phase, the vortices, which are lowpressure areas, are convected downstream at the local speed of the base flow. They finally impact the downstream cavity edge and generate an infinitely fast pressure wave which subsequently excites the shear layer at the upstream edge of the cavity. After $t \approx 2$, the pressure signal decreases continuously, and the pressure feedback between the downstream and upstream cavity edge diminishes significantly. Within the linear framework the actuator now generates vortical structures that annihilate the uncontrolled flow field (i.e. the unstable global mode). At the end, the impact of vortical structures on the downstream edge of the cavity is substantially suppressed which in effect eliminates any pressure feedback. From a physical point of view then, the control acts to break the closed pressure feedback-loop that is responsible for the global instability of the uncontrolled flow. 


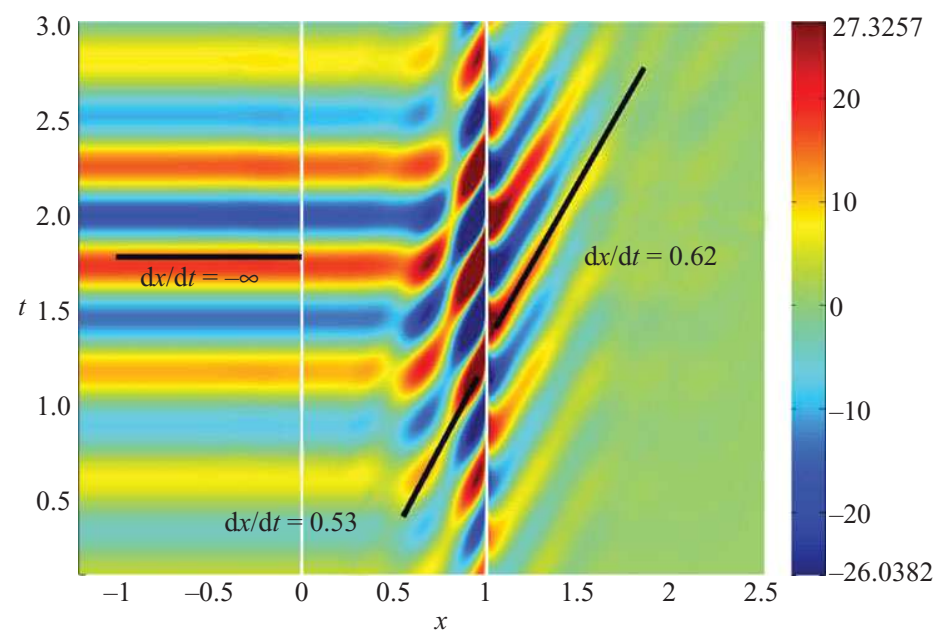

FIGURE 17. Spatio-temporal $x$ - $t$ diagram of pressure $p(x, y=0, t)$ contours with "best control strategy" applied. The initial condition consists of the most unstable global mode. The two vertical white lines indicate the cavity walls. See also movie II in the online version of the paper.

It is interesting to see that even when the 'best control strategy' is considered, a certain amount of transient growth is still observed. It is a consequence of the time it takes to estimate the flow state as well as the convective time for the control to be felt. Roughly speaking, the estimator produces a good approximation of the flow field only for $t>1$, and therefore the control law $c(t)$ which is fed back to the numerical simulation via the controller is only effective for $t>1$. Vortical structures are then created at the leading edge of the cavity and need approximately $t \approx 1$ to act on the most energetic perturbations located at the trailing edge of the cavity. Thus, the total time before a decrease of perturbation energy due to partial-state measurement control can be expected to be $t \approx 2$.

From these results we conclude that the compensator works optimally if the estimator is provided with the projected measurement $\hat{m}(t)$. To highlight the difference between the full measurement $m$ and the projected (or reduced) measurement $\hat{m}$ we plot the two quantities versus time for the best control strategy with $p=0$ (only eight unstable global modes are used in the reduced-order model) in figure 18( $a)$. We notice that the true measurement $m(t)$ (green line) is significantly different from the projected measurement $\hat{m}(t)$ (black dashed line), particularly in the range $0<t<3$. This fact explains the failure of partial-state control with only unstable global modes contained in the reduced-order model. Note also that for $0<t<1.7$ the full measurement $m$ is equal to the measurement given by the uncontrolled case (red line). Since the reducedorder model in this case is only based on the unstable global modes, this shows that the stable subspace is excited in a manner that keeps the complete measurement unchanged (see Barbagallo et al. 2008).

In addition, this demonstrates that, if the full measurement is to be fed into the estimator (as for partial-state control), the stable subspace needs to be modelled such that the full measurement can be satisfactorily approximated by the reducedorder model. In figure $18(b)$, it is shown that for partial-state simulations based on a reduced-order model with 13 BPOD modes, the full measurement (green solid line) and the projected measurement $\hat{m}$ (dashed line) agree. When this agreement does not exist, the following arguments furnish an explanation for the failure of the 

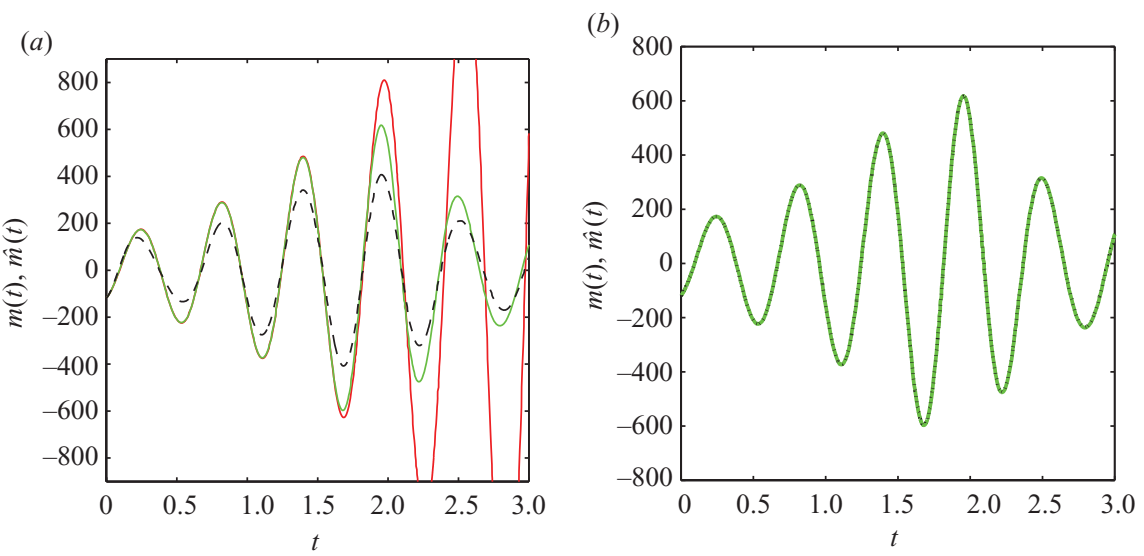

FIGURE 18. Measurement as a function of time using a reduced model based on the unstable global modes with "best control strategy" $(a)$ and using a reduced model based on unstable global modes and 13 BPOD modes with partial-state control $(b)$. Comparison of the true measurement signal (green solid line) with the measurement signal obtained from projecting onto a reduced basis consisting of the unstable global modes and 13 balanced POD modes (black dashed line). Red solid line: full measurement of uncontrolled simulation.

compensator to stabilize the flow: the actuator acts on the modes of the reduced-order model in order to suppress the instabilities but, by doing so, also excites flow states outside the range of the reduced-order model. These states are in turn detected by the sensor and cause a discrepancy between $m$ and $\hat{m}$ which, as demonstrated above, results in an unstable system.

As reported by Ehrenstein \& Gallaire (2008), if the control action is restricted to the space spanned by the modes of the reduced-order model, the additional structures (outside the range of the reduced-order model) alluded to above do not arise, and the compensator will be effective in rendering the system stable.

\subsubsection{Performance of a reduced model based on global modes}

For our flow configuration it has been demonstrated (see $\S 5.2$ ) that an expansion in global modes did not succeed in approximating the exact transfer function to a sufficient degree of accuracy; and it is natural to assume that this shortcoming will also have consequence for the performance of a reduced-order controller based on global modes. Nevertheless, this plan of action has been followed by Åkervik et al. (2007) and Ehrenstein \& Gallaire (2008) where least stable eigenmodes have been added to augment the expansion basis of the reduced-order model. The same strategy has also been attempted in our case with reduced-order models including up to a few thousand global modes. Alas, a stabilization of the flow could not be obtained, which indicates that the expansion of the stable subspace by the least stable modes does not always yield satisfactory results. This is in agreement with the open-loop behaviour of reduced-order models based on stable global modes which has been found inadequate to approximate the transfer function of the stable subspace.

\subsection{Analysis of the performance of the compensated system using stability analysis}

The stability of the compensated system has been already alluded to earlier as a desired and necessary property of a successful control design. In this section, we introduce an alternative way of studying the stability of the compensated system by taking advantage of the fact that the input-output behaviour of the numerical 
simulation (DNS) can be accurately captured by a low-order system including eight unstable global modes (which model the unstable subspace) and 13 BPOD modes for the stable subspace. This special configuration was chosen since it corresponds to the lowest $\mathscr{H}_{\infty}$-error norm (see figure 9). The resulting proxy system will be particularly advantageous, as far as computational efforts are concerned, for our continued investigation and analysis of the stability of the compensated problem using various reduced-order models.

In what follows, we assume that the partial-state simulation is governed by the following system of equations (see (6.6))

$$
\begin{aligned}
& \frac{\mathrm{d}}{\mathrm{d} t}\left(\begin{array}{c}
\hat{\boldsymbol{X}}_{\tilde{n}, 1} \\
\hat{\boldsymbol{Y}}_{\tilde{p}, 1}
\end{array}\right)=\left(\begin{array}{cc}
\hat{\boldsymbol{A}}_{\tilde{n}, \tilde{n}} & \hat{\boldsymbol{C}}_{\tilde{n}, 1} \hat{\boldsymbol{K}}_{1, \tilde{p}} \\
-\hat{\boldsymbol{L}}_{\tilde{p}, 1} \hat{\boldsymbol{M}}_{1, \tilde{n}} & \hat{\boldsymbol{A}}_{\tilde{p}, \tilde{p}}+\hat{\boldsymbol{C}}_{\tilde{p}, 1} \hat{\boldsymbol{K}}_{1, \tilde{p}}+\hat{\boldsymbol{L}}_{\tilde{p}, 1} \hat{\boldsymbol{M}}_{1, \tilde{p}}
\end{array}\right)\left(\begin{array}{c}
\hat{\boldsymbol{X}}_{\tilde{n}, 1} \\
\hat{\boldsymbol{Y}}_{\tilde{p}, 1}
\end{array}\right), \\
&\left.\left(\begin{array}{c}
\hat{\boldsymbol{X}}_{\tilde{n}, 1} \\
\hat{\boldsymbol{Y}}_{\tilde{p}, 1}
\end{array}\right)\right|_{t=0}=\left(\begin{array}{c}
\boldsymbol{W} \boldsymbol{Q} \boldsymbol{X}(0) \\
0
\end{array}\right),
\end{aligned}
$$

where $\tilde{n}=13+8$ and $\tilde{p}=p+8$ correspond to the total size of the plant and the estimator. The true measurement is obtained through $m(t)=\hat{\boldsymbol{M}}_{1, \tilde{n}} \hat{\boldsymbol{X}}_{\tilde{n}, 1}$. We thus have $\hat{\boldsymbol{A}}_{\tilde{n}, \tilde{n}}, \hat{\boldsymbol{C}}_{\tilde{n}, 1}, \hat{\boldsymbol{M}}_{1, \tilde{n}}$ which remain unchanged since they represent the dynamics of the DNS. On the other hand, $\hat{\boldsymbol{A}}_{\tilde{p}, \tilde{p}}, \hat{\boldsymbol{C}}_{\tilde{p}, 1}, \hat{\boldsymbol{M}}_{1, \tilde{p}}$ stand for the reduced matrices in the $\tilde{p}$ dimensional reduced basis. In short, the plant is a reduced-order model based on eight unstable global modes and 13 BPOD modes whereas the estimator is based on eight unstable global modes and $p$ stable modes (considering successively BPOD modes, POD modes and stable global modes). The eigenvalues of the coupled matrix which appears in (6.12) may be analysed for different values of $p$, indicating whether the compensated problem is stable or unstable.

\subsubsection{Stability of a reduced-order model based on BPOD modes}

In figure 19 we depict the spectrum of the coupled matrix for two cases, taking $p=0$ and $p=13$. In the former case $(p=0)$ we obtain five unstable eigenvalues in the half-plane given by $\omega \geqslant 0$ (the plot is symmetric with respect to $\omega=0$ ) which leads to the conclusion that the system with an estimator based solely on the unstable global modes is strongly unstable. As the number $p$ of BPOD modes increases, the eigenvalues move towards the stable half-plane. The limiting case is reached for $p=13$ when we retrieve the stable spectrum of the reduced model based on 13 BPOD modes (displayed with black circles in figure 10) plus the four double eigenvalues that result from the reflection of the four unstable global modes about the neutral stability line (four for the estimator and four for the controller).

An interesting feature worth pointing out concerns the non-uniform sensitivity of the eigenvalue location to the quality of the estimator. For example, the eigenvalue located at $(\sigma, \omega) \approx(-0.0324,16.73)$ is only weakly affected by a decreasing number $p$ of included BPOD modes, whereas the eigenvalue located at $(\sigma, \omega) \approx(-0.890,10.9)$ for $p=13$ protrudes far into the unstable half-plane as $p$ is reduced. This underlines the fact that robustness of a closed-loop system cannot simply be defined by its least damped eigenvalue.

In figure 20 the amplification rate $\sigma_{\max }$ of the most unstable eigenvalue of the coupled system as a function of the number of considered modes $p$ is shown. For $p=0$ (not shown in figure 20), we find that $\sigma_{\max }=0.53$ which shows again that a partialstate simulation with a reduced-order model including only the eight unstable global modes is strongly unstable. It is interesting to note that as soon as the control is active 


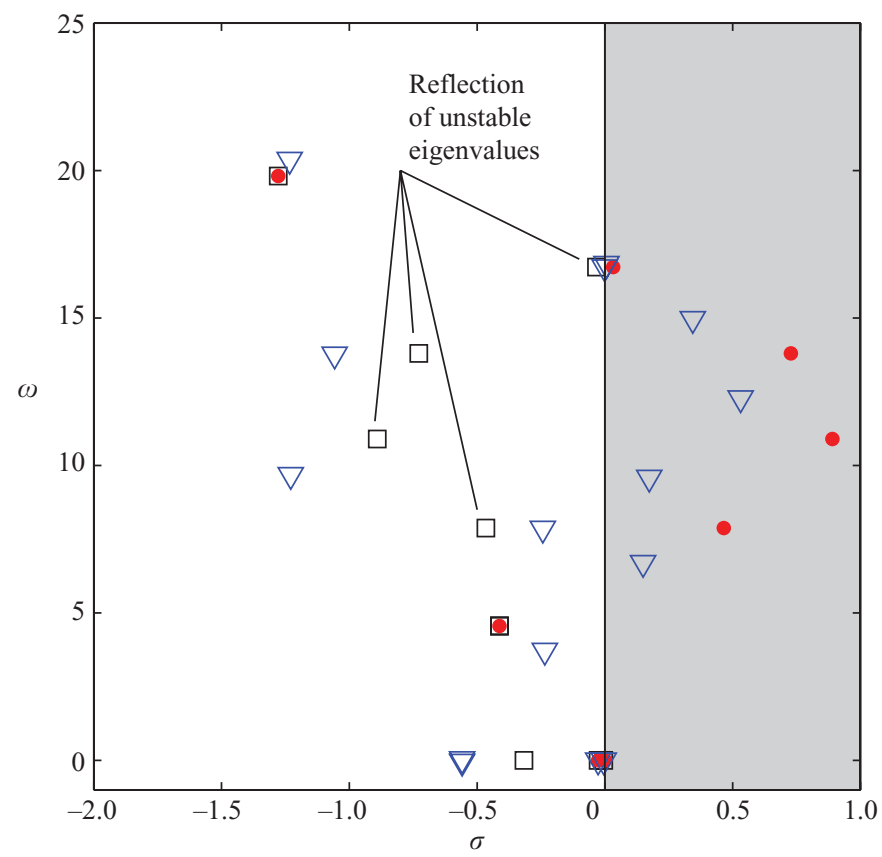

FIGURE 19. Global spectrum of the compensated system for $p=0$ (triangles) and $p=13$ (squares). Red dots correspond to the uncontrolled plant, i.e. eigenvalues of $\hat{\boldsymbol{A}}_{\tilde{n}, \tilde{n}}$.

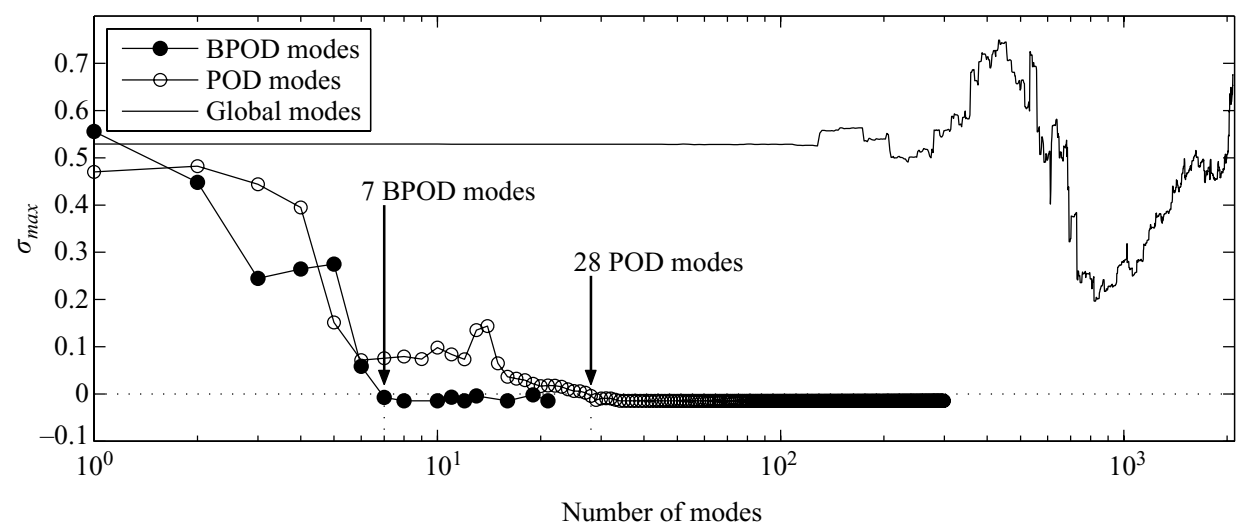

FIGURE 20. Least stable eigenvalue of the compensated system versus the number of included stable modes for BPOD modes (full symbols), POD modes (open circles) and global modes (solid line).

(after $t=2$ ) the slope of the energy corresponding to this case (see figure 16) is equal to $2 \sigma_{\max }$. For $p=13 \mathrm{BPOD}$ modes, the amplification rate is $\sigma_{\max }=-0.0144$ which is in agreement with the spectra presented in figure 19. Moreover, the partial-state control based on BPOD modes remains unstable up to $p=6$ suggesting a minimum of seven BPOD modes to stabilize closed-loop simulations. For values of $p \geqslant 7$, the simulations remain stable, and the amplificate rate of the least stable eigenmode converges towards $\sigma_{\max }=-0.0144$, a value which is close to the amplification rate $\sigma=-0.00811$ of the least stable eigenmode of the full system. 


\subsubsection{Stability of a reduced-order model based on POD modes}

We now turn our attention to partial-state control systems with estimators that use reduced-order models based on the eight unstable global modes and $p$ POD modes. The procedure is analogous to the one described in $\$ 6.4 .1$ in that, for each number $p$ of POD modes incorporated into the reduced-order model, we compute the eigenvalues of the coupled system (6.12). Results are shown in figure 20 (open circles) where we can see that a POD basis yields an effective estimator once at least 28 POD modes are included in the reduced-order model of the estimator. This is deduced from the fact that the amplification rate $\sigma_{\max }$ of the most unstable eigenvalue of (6.12) is positive for $0 \leqslant p \leqslant 27$ and negative for $p \geqslant 28$. Based on these results we conclude that the POD basis is suboptimal compared to the BPOD basis. Indeed, only seven BPOD modes are needed to arrive at a successful partial-state control system using a reduced-order model, whereas 28 POD modes are necessary to accomplish the same goal.

\subsubsection{Stability of a reduced-order model based on global modes}

The results of this analysis are given by the solid-line curve in figure 20. For the case $p=0$, i.e. if the reduced-order model includes only the eight unstable global modes, the partial-state control simulation is unstable, and the amplification rate $\sigma_{\max }$ is equal to 0.53 , as previously shown. If we then add to the estimator $p$ least stable global modes, the coupled system becomes more unstable (for $p=400$, the amplification rate rises up to $\sigma_{\max }=0.75$ ). This behaviour is followed by a drastic decrease in the amplification rate down to a level of $\sigma_{\max } \approx 0.2$ which is obtained using 750 least stable eigenmodes. This result demonstrates that adding more global modes to the estimator improves the coupled system since $\sigma_{\max }$ decreased; at no point, though, do we reach a stable coupled system with $\sigma_{\max }<0$. As a matter of fact, by including even more stable global modes in the reduced-order model of the estimator the performance of the coupled system deteriorates yet again, as is evident by the increasing amplification rate $\sigma_{\max }$.

\section{Summary and conclusions}

The incompressible flow over an open square cavity has been studied as a representative example of separated flows. At sufficiently high Reynolds numbers the shear layer atop the cavity exhibits a global instability. It is of general interest to devise effective control strategies to suppress this instability by feeding back measurement signals (taken downstream of the cavity) to actuators which manipulate the flow upstream of the cavity. The design of these control schemes quickly becomes computationally unfeasible due to the complexity of the problem and the resulting size of the matrix equations for the control and estimation gains. A model reduction has to be performed to bring back the construction of a compensated system into the realm of direct design methods. The basis underlying this reduction is a component that is pivotal to the success of the computed control scheme. A general bi-orthogonal projection framework has been developed that allows the analysis of different reduction bases.

The use of global modes for model reduction (see Ehrenstein \& Gallaire 2005, 2008; Åkervik et al. 2007; Henningson \& Åkervik 2008) is common despite the fact that this type of basis may be insufficient and inefficient in representing the inputoutput behaviour of the compensated system. Even though in Akervik et al. (2007) the studied flow configuration resulted in a stable compensated system based on global 
modes, our configuration provides a counter example which suggests that reducedorder modelling based on global modes cannot be generalized to more complex geometry and/or flow physics. In our case, this has been demonstrated by the lack of convergence of reduced transfer functions (based on global mode expansions) towards the exact one. The difficulties of expressing the transfer characteristics between sensor and actuator stems from the non-normality of the linearized Navier-Stokes equations. As a consequence, global modes with a substantial decay rate can still contribute to the input-output behaviour, while weakly damped global modes may not. In other words, the decay rate of global modes is a poor criterion for the inclusion of global modes in a projection basis or in the truncation of the projection basis. Rather, a criterion that accounts for the controllability and observability of each global mode, in addition to its decay rate, is more accurate in assessing which global mode contributes to what extent to the input-output behaviour.

The choice of a more appropriate expansion basis has to address the issues of controllability and observability which is essential in accurately representing the relation between actuator input and sensor output. Balanced modes are designed to accomplish this task by paying equal emphasis to controllability and observability and are thus ideally suited for the low-dimensional representation of the inputoutput behaviour. Despite this property, their practical computation from numerical simulation poses challenges that take significant effort to overcome. These challenges are mainly related to stability issues of the reduced system which, in turn, is related to the slow convergence of the approximate Gramians towards their exact solution when fluid motion on a slow time scale (such as for modes that have a significant component inside the cavity) is present and needs to be resolved. In this case, excessively long sequences of snapshots are necessary which puts strain on computational resources and memory requirements.

POD modes are often used as a technique to arrive at a reduced-order model. They overemphasize controllability at the expense of observability, but are still capable of expressing the input-output behaviour albeit not as efficiently as balanced modes. Their advantage, however, lies in the simplicity of computing them: a simple snapshot method suffices. In our case, reduced-order models based on POD modes, even though higher-dimensional than models based on BPOD modes, showed improved robustness when used in the design of reduced-order controllers. The approximation of the openloop transfer function by POD modes shows adequate convergence towards the exact transfer function, even though, compared to balanced modes, many more POD modes are necessary to achieve the same level of accuracy, as measured in the $\mathscr{H}_{\infty^{-}}$ norm.

In summary, both BPOD and POD modes are suitable choices for reducing the input-output relation of a control problem to a manageable size, where a choice has to be made between (i) an expansion in as few modes as possible, in which case BPOD modes prevail, and (ii) a somewhat more robust computational procedure to determine a larger reduced-order model, in which case POD modes appear to be a reasonable choice.

One should keep in mind, though, that flow over an open cavity - with the sensor located in the wake of the actuator - is particularly amenable to a reduced-order controller based on POD modes since the flow structures identified by the POD reflect the convective transport between the control and measurement device and thus are able to represent the input-output behaviour of the compensated system. For more challenging configurations, where this convective link between actuator and sensor is not given, the discrepancy between POD and BPOD modes may be drastically 
higher, and balanced modes may markedly surpass POD modes in their ability and efficiency in rendering the flow stable by reduced-order control schemes.

We like to thank Olivier Marquet and François Gallaire for helpful discussions and comments during the course of this work. The third author gratefully acknowledges financial support from the 'chaires d'excellence' programme of the Agence Nationale de la Recherche (ANR). The staff at the Café Daguerre is thanked for indulging our many animated discussions and work sessions.

\section{Appendix. Reduced-order models based on balanced proper orthogonal decomposition}

\section{A.1. Projected equations}

In this section, we will show that the system of equations (5.1) which governs the dynamics of the stable subspace can be written in the classical state-space form (defined in (5.10)).

Equation (5.1) can be cast in the form

$$
\begin{aligned}
\left(\begin{array}{cc}
\boldsymbol{Q}_{1} & 0 \\
0 & 0
\end{array}\right) \frac{\mathrm{d}}{\mathrm{d} t}\left(\begin{array}{l}
\boldsymbol{X}_{1} \\
\boldsymbol{X}_{2}
\end{array}\right) & =\left(\begin{array}{cc}
\boldsymbol{A}_{s 1} & \boldsymbol{A}_{s 2}^{*} \\
\boldsymbol{A}_{s 2} & 0
\end{array}\right)\left(\begin{array}{l}
\boldsymbol{X}_{1} \\
\boldsymbol{X}_{2}
\end{array}\right)+\left(\begin{array}{cc}
\boldsymbol{Q}_{1} & 0 \\
0 & 0
\end{array}\right)\left(\begin{array}{l}
\boldsymbol{C}_{s 1} \\
\boldsymbol{C}_{s 2}
\end{array}\right) c, \\
m & =\left(\begin{array}{ll}
\boldsymbol{M}_{1} & 0
\end{array}\right)\left(\begin{array}{l}
\boldsymbol{X}_{1} \\
\boldsymbol{X}_{2}
\end{array}\right),
\end{aligned}
$$

where $\boldsymbol{X}_{1}$ denotes the velocity fields and $\boldsymbol{X}_{2}$ stands for the corresponding pressure field. In what follows, the subscript ${ }_{1}$ applied to a vector field will denote the part of the state vector composed of the velocity field only. For the design of control and model reduction schemes we need to reformulate the above equations into standard state-space form. To this end we multiply the momentum equation by $\boldsymbol{A}_{s 2} \boldsymbol{Q}_{1}^{-1}$, which yields - assuming that $\boldsymbol{A}_{s 2} \dot{\boldsymbol{X}}_{1}=0$ - an expression for the pressure in terms of the velocity field

$$
\boldsymbol{X}_{2}=-\left(\boldsymbol{A}_{s 2} \boldsymbol{Q}_{1}^{-1} \boldsymbol{A}_{s 2}^{*}\right)^{-1}\left[\left(\boldsymbol{A}_{s 2} \boldsymbol{Q}_{1}^{-1} \boldsymbol{A}_{s 1}\right) \boldsymbol{X}_{1}+\boldsymbol{A}_{s 2} \boldsymbol{C}_{s 1}\right] .
$$

This relation can be used to eliminate the explicit divergence constraint and allows us to write the governing linearized equations in the desired form

$$
\begin{aligned}
\frac{\mathrm{d} \boldsymbol{X}_{1}}{\mathrm{~d} t} & =\boldsymbol{P}_{1} \boldsymbol{A}_{s 1} \boldsymbol{X}_{1}+\boldsymbol{P}_{1} \boldsymbol{Q}_{1} \boldsymbol{C}_{s 1} c, \\
m & =\boldsymbol{M}_{1} \boldsymbol{X}_{1},
\end{aligned}
$$

where

$$
\boldsymbol{P}_{1} \boldsymbol{Q}_{1}=\boldsymbol{I}-\boldsymbol{Q}_{1}^{-1} \boldsymbol{A}_{s 2}^{*}\left(\boldsymbol{A}_{s 2} \boldsymbol{Q}_{1}^{-1} \boldsymbol{A}_{s 2}^{*}\right)^{-1} \boldsymbol{A}_{s 2}
$$

is the projection matrix onto the divergence-free space. This projector is reminiscent of the steps taken by a projection method to correct a preliminary divergent velocity field towards a solenoidal one. Starting from the right of the above expression, we take the divergence $\left(\boldsymbol{A}_{s 2}\right)$ of the velocity field, then solve a Poisson equation $\left(\left(\boldsymbol{A}_{s 2} \boldsymbol{Q}_{1}^{-1} \boldsymbol{A}_{s 2}^{*}\right)^{-1}\right)$ for the corrective pressure, and finally take the gradient of the pressure $\left(\boldsymbol{Q}_{1}^{-1} \boldsymbol{A}_{s 2}^{*}\right)$ and subtract this component from the preliminary velocity field. This procedure then renders the velocity field divergence free. In short, if $\boldsymbol{X}_{1}$ is a non-divergence-free velocity field, then $\boldsymbol{P}_{1} \boldsymbol{Q}_{1} \boldsymbol{X}_{1}$ is the projection of $\boldsymbol{X}_{1}$ onto the divergence-free subspace; if $\boldsymbol{X}_{1}$ is already divergence free, we have $\boldsymbol{P}_{1} \boldsymbol{Q}_{1} \boldsymbol{X}_{1}=\boldsymbol{X}_{1}$. It is important to point out 
that the projector $\boldsymbol{P}_{1} \boldsymbol{Q}_{1}$ is also used to properly adjust an initial condition which does not satisfy $\boldsymbol{u} \cdot \boldsymbol{n}=0$ along some boundaries like solid walls. It is equally noteworthy that $\boldsymbol{P}_{1}$ is a Hermitian operator, so that we can take advantage of the relation $\boldsymbol{P}_{1}^{*}=\boldsymbol{P}_{1}$.

Defining $\boldsymbol{A}_{1}=\boldsymbol{P}_{1} \boldsymbol{A}_{s 1}$ and $\boldsymbol{C}_{1}=\boldsymbol{P}_{1} \boldsymbol{Q}_{1} \boldsymbol{C}_{s 1}$, we recover (5.10).

\section{A.2. Controllability Gramian}

We consider the controllability Gramian $\boldsymbol{G}_{C}$ defined, for our system, as

$$
\boldsymbol{G}_{C}=\int_{0}^{\infty} \mathrm{e}^{\boldsymbol{A}_{1} t} \boldsymbol{C}_{1} \boldsymbol{C}_{1}^{*} \mathrm{e}^{\mathbf{A}_{1}^{*} t} \mathrm{~d} t
$$

Let us consider a fully controllable system, which means that all divergence-free states $\boldsymbol{X}_{1}$ may be reached by a control law $c(t)$. Furthermore, let $\boldsymbol{X}_{1}$ be a divergence-free field. We will demonstrate that the minimum energy $\int_{0}^{\infty} c^{*}(t) c(t) \mathrm{d} t$ to reach $\boldsymbol{X}_{1}$ is equal to $\boldsymbol{X}_{1}^{*} \boldsymbol{G}_{C}^{-1} \boldsymbol{X}_{1}$. We know that there exists a unique vector $\boldsymbol{\xi}$ such that $\boldsymbol{X}_{1}=\boldsymbol{G}_{C} \boldsymbol{\xi}$ is satisfied and note that $\boldsymbol{\xi}=\boldsymbol{G}_{C}^{-1} \boldsymbol{X}_{1}$, even though the matrix $\boldsymbol{G}_{C}$ may be degenerate. After considering the control law $c(t)=\boldsymbol{C}_{1}^{*} \mathrm{e}^{\boldsymbol{A}_{1}^{*} \boldsymbol{\xi}}$, it may easily be verified that this control law yields $\boldsymbol{X}_{1}$ as $t \rightarrow \infty$

$$
\int_{0}^{\infty} \mathrm{e}^{\boldsymbol{A}_{1} t} \boldsymbol{C}_{1} c(t) \mathrm{d} t=\boldsymbol{X}_{1}
$$

One may furthermore show that this specific $c(t)$ yields the minimum energy to reach $\boldsymbol{X}_{1}$. This minimum energy reads

$$
\begin{aligned}
\int_{0}^{\infty} c^{*}(t) c(t) \mathrm{d} t & =\xi^{*} \int_{0}^{\infty} \mathrm{e}^{\boldsymbol{A}_{1} t} \boldsymbol{C}_{1} \boldsymbol{C}_{1}^{*} \mathrm{e}^{\boldsymbol{A}_{1}^{*} t} \mathrm{~d} t \boldsymbol{\xi} \\
& =\boldsymbol{\xi}^{*} \boldsymbol{G}_{C} \boldsymbol{\xi} \\
& =\boldsymbol{X}_{1}^{*} \boldsymbol{G}_{C}^{-1} \boldsymbol{X}_{1}
\end{aligned}
$$

which verifies our proposition above.

The controllability Gramian $\boldsymbol{G}_{C}$ can be computed by solving a matrix Lyapunov equation (see Antoulas 2005); its actual solution by standard numerical techniques, however, quickly becomes prohibitively demanding on computational resources and algorithms. Instead, the Gramian can be approximated by a discrete integration in time according to

$$
\begin{aligned}
\boldsymbol{G}_{C} & =\int_{0}^{\infty} \boldsymbol{X}_{1}(t) \boldsymbol{X}_{1}^{*}(t) \mathrm{d} t, \\
& \approx \sum_{i \geqslant 0} \boldsymbol{X}_{1}\left(t_{i}\right) \boldsymbol{X}_{1}^{*}\left(t_{i}\right) \delta_{i}
\end{aligned}
$$

with $\boldsymbol{X}_{1}(t)=\mathrm{e}^{\boldsymbol{A}_{1} t} \boldsymbol{C}_{1}$ and $\delta_{i}$ denoting appropriate quadrature coefficients. The flow fields $\boldsymbol{X}_{1}\left(t_{i}\right)$ at discrete instances $t_{i}$ can be obtained by the integration in time of the linear system

$$
\begin{aligned}
\dot{\boldsymbol{X}}_{1} & =\boldsymbol{A}_{1} \boldsymbol{X}_{1}, \\
\boldsymbol{X}_{1}(0) & =\boldsymbol{C}_{1}
\end{aligned}
$$

or

$$
\left(\begin{array}{cc}
\boldsymbol{Q}_{1} & 0 \\
0 & 0
\end{array}\right) \frac{\mathrm{d}}{\mathrm{d} t}\left(\begin{array}{l}
\boldsymbol{X}_{1} \\
\boldsymbol{X}_{2}
\end{array}\right)=\left(\begin{array}{cc}
\boldsymbol{A}_{s 1} & \boldsymbol{A}_{s 2}^{*} \\
\boldsymbol{A}_{s 2} & 0
\end{array}\right)\left(\begin{array}{l}
\boldsymbol{X}_{1} \\
\boldsymbol{X}_{2}
\end{array}\right), \quad \boldsymbol{X}_{1}(0)=\boldsymbol{P}_{1} \boldsymbol{Q}_{1} \boldsymbol{C}_{1} .
$$

This system, corresponding to the direct problem ( $\mathrm{cf}(5.1)$ ), can straightforwardly be solved by our linearized DNS programme. As a result, the controllability Gramian 
may therefore be written as

$$
\mathbf{G}_{C}=\boldsymbol{T}_{1} \boldsymbol{T}_{1}^{*}
$$

where

$$
\boldsymbol{T}=\left[\begin{array}{lll}
\boldsymbol{X}(0 \Delta t) \sqrt{\delta_{0}} & \boldsymbol{X}(1 \Delta t) \sqrt{\delta_{1}} \ldots
\end{array}\right]
$$

Although we use an equidistant spacing between the snapshots, a non-equidistant spacing between them is conceivable to increase the accuracy of the integration in time or to account for localized features that require higher temporal resolution. The dimension of $\boldsymbol{T}$ is $n \times n_{d}$ where $n$ and $n_{d}$ denote the number of degrees of freedom in $\boldsymbol{X}$ and the number of direct snapshots, respectively. Note that $\boldsymbol{T}_{1}$ which represents the velocity components of $\boldsymbol{T}$ is of dimension $n_{1} \times n_{d}$ with $n_{1}$ as the number of degrees of freedom of the velocity field. The quadrature coefficients $\delta_{i}$ correspond to the fourth-order Simpson method.

The above demonstration shows that the controllability Gramian $\boldsymbol{G}_{C}$ can be thought of as the spatial correlation matrix for the evolution of an impulsive forcing at the actuator location (the initial condition is $\mathbf{P} \mathbf{Q}_{1} \boldsymbol{C}_{1}$ ). We further conclude that the eigenvectors of $\boldsymbol{G}_{C} \boldsymbol{Q}_{1}$ are the commonly used POD modes for our particular initial condition. These POD modes represent flow structures that are easily triggered by control input since they require rather small amounts of control energy to be excited.

\section{A.3. Observability Gramian}

The observability Gramian is defined as

$$
\boldsymbol{G}_{O}=\int_{0}^{\infty} \mathrm{e}^{\mathbf{A}_{1}^{t} t} \boldsymbol{M}_{1}^{*} \boldsymbol{M}_{1} \mathrm{e}^{\boldsymbol{A}_{1} t} \mathrm{~d} t .
$$

With $\boldsymbol{X}_{1}$ denoting a divergence-free flow field, we will show that the measurement energy is equal to $\int_{0}^{\infty} m^{*}(t) m(t) \mathrm{d} t=\boldsymbol{X}_{1}^{*} \boldsymbol{G}_{O} \boldsymbol{X}_{1}$. The response of the sensor to our initial state $\boldsymbol{X}_{1}$ is simply given by $m(t)=\boldsymbol{M} \mathrm{e}^{\boldsymbol{A}_{1} t} \boldsymbol{X}_{1}$, and the following expression

$$
\begin{aligned}
\int_{0}^{\infty} m^{*}(t) m(t) \mathrm{d} t & =\int_{0}^{\infty} \boldsymbol{X}_{1}^{*} \mathrm{e}^{\boldsymbol{A}_{1}^{*} t} \boldsymbol{M}_{1}^{*} \boldsymbol{M}_{1} \mathrm{e}^{\boldsymbol{A}_{1} t} \boldsymbol{X}_{1} \mathrm{~d} t, \\
& =\boldsymbol{X}_{1}^{*} \boldsymbol{G}_{O} \boldsymbol{X}_{1}
\end{aligned}
$$

corroborates our statement above.

Analogous to the previous section, the observability Gramian $\boldsymbol{G}_{O}$ can only be meaningfully defined for stable linear systems and can then be determined as the solution of a matrix Lyapunov equation. For the same reason as above, however, the solution based on a Lyapunov equation becomes excessively expensive, thus prompting us to compute an approximation of the observability Gramian based on a discrete integration in time. $\boldsymbol{G}_{O}$ can be rewritten as

$$
\begin{aligned}
\mathbf{G}_{O} & =\int_{0}^{\infty} \boldsymbol{Q}_{1} \boldsymbol{P}_{1} \mathrm{e}^{\boldsymbol{A}_{s 1}^{*} \boldsymbol{P}_{1} t} \boldsymbol{M}_{1}^{*} \boldsymbol{M}_{1} \mathrm{e}^{\boldsymbol{P}_{1} \boldsymbol{A}_{s 1} t} \boldsymbol{P}_{1} \mathbf{Q}_{1} \mathrm{~d} t \\
& =\mathbf{Q}_{1}\left(\int_{0}^{\infty} \mathrm{e}^{\boldsymbol{P}_{1} \boldsymbol{A}_{s 1}^{*} t} \boldsymbol{P}_{1} \mathbf{Q}_{1}\left(\boldsymbol{Q}_{1}^{-1} \boldsymbol{M}_{1}^{*}\right) \boldsymbol{M}_{1} \boldsymbol{Q}_{1}^{-1} \boldsymbol{Q}_{1} \boldsymbol{P}_{1} \mathrm{e}^{\boldsymbol{A}_{s 1} \boldsymbol{P}_{1} t} \mathrm{~d} t\right) \boldsymbol{Q}_{1} \\
& =\boldsymbol{Q}_{1}\left(\int_{0}^{\infty} \boldsymbol{Y}_{1}(t) \boldsymbol{Y}_{1}^{*}(t) \mathrm{d} t\right) \boldsymbol{Q}_{1} \\
& \approx \mathbf{Q}_{1}\left(\sum_{i \geqslant 0} \boldsymbol{Y}_{1}\left(t_{i}\right) \boldsymbol{Y}_{1}^{*}\left(t_{i}\right) \delta_{i}\right) \mathbf{Q}_{1}
\end{aligned}
$$


where $\boldsymbol{Y}_{1}(t)=\mathrm{e}^{\boldsymbol{P}_{1} \boldsymbol{A}_{s 1}^{*} t} \boldsymbol{P}_{1} \boldsymbol{Q}_{1}\left(\boldsymbol{Q}_{1}^{-1} \boldsymbol{M}_{1}^{*}\right)$ and $\delta_{i}$ denotes, as before, the quadrature coefficients for the evaluation of the time integral. The flow fields $\boldsymbol{Y}_{1}(t)$ can be obtained by integration in time of the adjoint system defined as

$$
\begin{aligned}
\frac{\mathrm{d} \boldsymbol{Y}_{1}}{\mathrm{~d} t} & =\boldsymbol{P}_{1} \boldsymbol{A}_{s 1}{ }^{*} \boldsymbol{Y}_{1}, \\
\boldsymbol{Y}_{1}(0) & =\boldsymbol{P}_{1} \boldsymbol{Q}_{1}\left(\boldsymbol{Q}_{1}^{-1} \boldsymbol{M}_{1}^{*}\right)
\end{aligned}
$$

or

$$
\left(\begin{array}{cc}
\boldsymbol{Q}_{1} & 0 \\
0 & 0
\end{array}\right) \frac{\mathrm{d}}{\mathrm{d} t}\left(\begin{array}{c}
\boldsymbol{Y}_{1} \\
\boldsymbol{Y}_{2}
\end{array}\right)=\left(\begin{array}{cc}
\boldsymbol{A}_{s 1}^{*} & \boldsymbol{A}_{s 2}^{*} \\
\boldsymbol{A}_{s 2} & 0
\end{array}\right)\left(\begin{array}{c}
\boldsymbol{Y}_{1} \\
\boldsymbol{Y}_{2}
\end{array}\right), \quad \boldsymbol{Y}_{1}(0)=\boldsymbol{P}_{1} \boldsymbol{Q}_{1}\left(\boldsymbol{Q}_{1}^{-1} \boldsymbol{M}_{1}^{*}\right) .
$$

This adjoint initial-value problem is solved by our adjoint linearized numerical simulation code, i.e. (5.1) in which $\boldsymbol{A}$ is replaced by its transpose conjugate $\boldsymbol{A}^{*}$.

As a result, the observability Gramian may therefore be rewritten as

$$
\mathbf{G}_{O}=\mathbf{Q}_{1} \boldsymbol{U}_{1} \mathbf{U}_{1}^{*} \mathbf{Q}_{1}
$$

where

$$
\boldsymbol{U}=\left[\boldsymbol{Y}(0 \Delta t) \sqrt{\delta_{0}} \quad \boldsymbol{Y}(1 \Delta t) \sqrt{\delta_{1}} \ldots\right] .
$$

The dimension of $\boldsymbol{U}$ is $n \times n_{a}$ where $n_{a}$ stands for the number of adjoint snapshots. The quadrature coefficients $\delta_{i}$ correspond again to the fourth-order Simpson method.

\section{REFERENCES}

Ahuja, S. \& Rowley, C. W. 2008 Low-dimensional models for feedback stabilization of unstable steady states. AIAA Paper 2008-553.

Åkervik, E., Hefpffner, J., Ehrenstein, U. \& Henningson, D. S. 2007 Optimal growth, model reduction and control in a separated boundary-layer flow using global modes. J. Fluid Mech. 579, 305-314.

Amestoy, P. R., Duff, I. S., Koster, J. \& L'Excellent, J.-Y. 2001 A fully asynchronous multifrontal solver using distributed dynamic scheduling. SIAM J. Matrix Anal. Appl. 23 (1), 15-41.

Antoulas, A. C. 2005 Approximation of Large-Scale Dynamical Systems. SIAM.

Antoulas, A., Sorensen, D. \& Gugercin, S. 2001 A survey of model reduction methods for large-scale systems. Contemp. Math. 280, 193-219.

Bagheri, S., Brandt, L. \& Henningson, D. S. $2009 a$ Input-output analysis, model reduction and control of the flat-plate boundary layer. J. Fluid Mech. 620, 263-298.

Bagheri, S., Hepffner, J., Schmid, P. J. \& Henningson, D. S. $2009 b$ Input-output analysis and control design applied to a linear model of spatially developing flows. Appl. Mech. Rev. 62 (2), 020803-1-27.

BAI, Z. 2002 Krylov subspace techniques for reduced-order modelling of large-scale dynamical systems. Appl. Numer. Math. 43, 9-44.

Barbagallo, A., Sipp, D., Jacquin, L. \& Schmid, P. J. 2008 Control of an incompressible cavity flow using a reduced model based on global modes. In Fifth AIAA Theoretical Fluid Mechanics Conference, Seattle. AIAA paper 2008-3904.

Barkley, D., Gomes, M. G. \& Henderson, R. D. 2002 Three-dimensional instability in flow over a backward-facing step. J. Fluid Mech. 473, 167-190.

Bergman, M., Cordier, L. \& Brancher, J.-P. 2006 Optimal rotary control of the cylinder wake using POD reduced-order model. Phys. Fluids 17, 305-314.

Berkooz, G., Holmes, P. \& Lumley, J. L. 1993 The proper orthogonal decomposition in the analysis of turbulent flows. Annu. Rev. Fluid Mech. 25, 539-575.

Bewley, T. R. 2001 Flow control: new challenges for a new renaissance. Prog. Aerosp. Sci. 37, $21-58$. 
Blackburn, H. M., Barkley, D. \& Sherwin, S. J. 2008 Convective instability and transient growth in flow over a backward-facing step. J. Fluid Mech. 603, 271-304.

Bres, G. A. \& Colonius, T. 2008 Three-dimensional instabilities in compressible flow over open cavities. J. Fluid Mech. 599, 309-339.

Buffoni, M., Camarri, S. \& Iollo, A. 2006 Low-dimensional modelling of a confined threedimensional wake flow. J. Fluid Mech. 569, 141-150.

BurL, J. B. 1999 Linear Optimal Control. $\mathscr{H}_{2}$ and $\mathscr{H}_{\infty}$ Methods. Addison-Wesley.

Cattafesta, L. N., Song, Q., Williams, D. R., Rowley, C. W. \& Alvi, F. 2008 Active control of flow-induced cavity oscillations. Prog. Aerosp. Sci. 44, 459-502.

Cattafesta, L. N., Williams, D. R., Rowley, C. W. \& Alvi, F. S. 2003 Review of active flow control of flow-induced cavity resonance. AIAA Paper 2003-3567 33rd AIAA Fluid Dyn. Conf., Orlando, FL, June 2003.

Chevalier, M., Heepffner, J., Åkervik, E. \& Henningson, D. S. 2007 Linear feedback control and estimation applied to instabilities in spatially developing boundary layers. J. Fluid Mech. 588, $163-187$.

Crouch, J. D., Garbaruk, A. \& Magidov, D. 2007 Predicting the onset of flow unsteadiness based on global instability. J. Comput. Phys. 224, 924-940.

Delville, J., Cordier, L. \& Bonnet, J. P. 1998 Large-scale structure identification and control in turbulent shear flows. In Flow Control: Fundamentals and Practice (eds. M. Gad-el-Hak, A. Pollard, J.-P. Bonnet) pp. 199-273. Springer.

Ding, Y. \& KawahaRA, M. 1999 Three-dimensional linear stability analysis of incompressible viscous flows using finite element method. Intl J. Numer. Meth. Fluids 31, 451-479.

Ehrenstein, U. \& Gallaire, F. 2005 On two-dimensional temporal modes in spatially evolving open flows. J. Fluid Mech. 536, 209-218.

Ehrenstein, U. \& Gallaire, F. 2008 Optimal perturbations and low-frequency oscillations in a separated boundary-layer flow. In Fifth AIAA Theoretical Fluid Mechanics Conference, Seattle. AIAA paper 2008-4232.

Faure, T. M., Adrianos, P., Lusseyran, F. \& Pastur, L. 2007 Visualizations of the flow inside an open cavity at medium-range Reynolds numbers. Exp. Fluids 42, 169-184.

Freund, R. 2003 Model reduction methods based on Krylov subspaces. Acta Numer. 12, 267-319.

Galletti, B., Bottaro, A., Bruneau, C. H. \& Iollo, A. 2007 Accurate model reduction of transient and forced wakes. Eur. J. Mech. B 26, 354-366.

Henningson, D. S. \& Åkervik, E. 2008 The use of global modes to understand transition and perform flow control. Phys. Fluids 20, 031302.

Heppffner, J., Chevalier, M., Bewley, T. R. \& Henningson, D. S. 2005 State estimation in wall-bounded flow systems. Part 1. Laminar flows. J. Fluid Mech. 534, 263-294.

Högberg, M., Bewley, T. R. \& Henningson, D. S. 2003 Linear feedback control and estimation of transition in plane channel flow. J. Fluid Mech. 481, 149-175.

ILAK, M. \& RowLEY, C. W. 2006 Reduced-order modelling of channel flow using travelling POD and balanced POD. In Third AIAA Flow Control Conference, San Francisco, AIAA paper 2006-3194.

ILAK, M. \& Rowley, C. W. 2008 Modelling of transitional channel flow using balanced proper orthogonal decomposition. Phys. Fluids 20, 034103.

JACKSON, C. P. 1987 A finite-element study of the onset of vortex shedding in flow past variously shaped bodies. J. Fluid Mech. 182, 23-45.

Joshi, S. S., SpeYer, J. L. \& KIM, J. 1997 A systems theory approach to the feedback stabilization of infinitesimal and finite-amplitude disturbances in plane Poiseuille flow. J. Fluid Mech. 332, $157-184$.

KIM, J. 2003 Control of turbulent boundary layers. Phys. Fluids 15, 1093-1105.

Kim, J. \& Bewley, T. R. 2007 A linear systems approach to flow control. Annu. Rev. Fluid Mech. 39, 383-417.

Lall, S., Marsden, J. E. \& Glavaski, S. 2002 A subspace approach to balanced truncation for model reduction of nonlinear control systems. Intl J. Robust Nonlinear Control 12, 519-535.

Lauga, E. \& Bewley, T. R. 2003 The decay of stabilizability with Reynolds number in a linear model of spatially developing flows. Proc. R. Soc. Lond. A 459, 2077-2095.

Lauga, E. \& Bewley, T. R. 2004 Performance of a linear robust control strategy on a nonlinear model of spatially developing flows. J. Fluid Mech. 512, 343-374. 
Lee, K. H., Cortelezzi, L., Kim, J. \& Speyer, J. 2001 Application of reduced-order controller to turbulent flows for drag reduction. Phys. Fluids 13, 1321-1330.

LehoucQ, R. B. \& Scott, J. A. 1997 Implicitly restarted Arnoldi methods and subspace iteration. SIAM J. Matrix Anal. Appl. 23, 551-562.

LehoucQ, R. B., Sorensen, D. C. \& YAng, C. 1998 ARPACK Users Guide: Solution of Large Scale Eigenvalue Problems with Implicitly Restarted Arnoldi methods. SIAM Publishing.

Lumley, J. L. 1970 Stochastic Tools in Turbulence. Academic Press.

Ma, Z., Ahuja, S. \& Rowley, C. W. Reduced order models for control of fluids using the eigensystem realization algorithm. Theor. Comput. Fluid Dyn. (submitted)

Marquet, O., Sipp, D., Chomaz, J.-M. \& Jacquin, L. 2008 Amplifier and resonator dynamics of a low-Reynolds-number recirculation bubble in a global framework. J. Fluid Mech. 605, 429-443.

Moin, P. \& Bewley, T. R. 1994 Feedback control of turbulence. Appl. Mech. Rev. 47 (6), S3-S13.

Moore, B. 1981 Principal component analysis in linear systems: controllability, observability, and model reduction. IEEE Trans. Autom. Control 26, 17-32.

Noack, B., Afanasiev, K., Morzynski, M., Tadmor, G. \& Thiele, F. 2003 A hierarchy of lowdimensional models for the transient and post-transient cylinder wake. J. Fluid Mech. 497, 335-363.

Noack, B. R. \& Eckelmann, H. 1994 A global stability analysis of the steady and periodic cylinder wake. J. Fluid Mech. 270, 297-330.

OR, A. C. \& Speyer, J. L. 2008 Model reduction of input-output dynamical systems by proper orthogonal decomposition. J. Guid. Control Dyn. 31-2, 322-328.

Podvin, B., Fraigneau, Y., Lusseyran, F. \& Gougat, P. 2006 A reconstruction method for the flow past an open cavity. ASME J. Fluids Engng 128, 531-540.

RowLEY, C. W. 2005 Model reduction for fluids using balanced proper orthogonal decomposition. Intl J. Bifurcation Chaos 15, 997-1013.

Rowley, C. W., Ahuja, S., Taira, K. \& Colonius, T. 2008 Closed-loop control of leading edge vorticity on $3 \mathrm{~d}$ wings: simulations and low-dimensional models. In Thirty-eighth Fluid Dynamics Conference and Exhibit, Seattle. AIAA paper 2008-3981.

Rowley, C. W. \& Williams, D. R. 2006 Dynamics and control of high-Reynolds-number flow over open cavities. Annu. Rev. Fluid Mech. 38, 251-276.

Samimy, M., Debiasi, M., Caraballo, E., Serrani, A., Yuan, X., Little, J. \& Myatt, J. 2007 Feedback control of subsonic cavity flows using reduced-order models. J. Fluid Mech. 579, 315-346.

SCHERPen, J. M. 1993 Balancing for nonlinear systems. Syst. Control Lett. 21, 143-153.

SipP, D. \& Lebedev, A. 2007 Global stability of base and mean flows: a general approach and its applications to cylinder and open cavity flows. J. Fluid Mech. 593, 333-358.

Sirovich, L. 1987 Turbulence and the dynamics of coherent structures. Q. Appl. Math. 45, 561-590.

Tadmor, G., Noack, B., Morzynski, M. \& Siegel, S. 2004 Low-dimensional models for feedback flow control. Part II. Control design and dynamical estimation. AIAA Paper 2004-2409 .

Theofilis, V. 2000 Advances in global linear instability analysis of nonparallel and three-dimensional flows. Prog. Aerosp. Sci. 39, 249-315.

Willcox, K. \& Peraire, J. 2002 Balanced model reduction via proper orthogonal decomposition. AIAA J. 40, 2323-2330.

ZebiB, A. 1987 Stability of a viscous flow past a circular cylindar. J. Engng Math. 21, 155-165.

Zhou, K., Salomon, G. \& Wu, E. 1999 Balanced realization and model reduction for unstable systems. Intl J. Robust Nonlinear Control 9, 183-198.

Zhou, K., Salomon, G. \& Wu, E. 2002 Robust and Optimal Control. Prentice Hall.

Zielinska, J. A. \& Wesfreid, J. E. 1995 On the spatial structure of global modes in wake flow. Phys. Fluids 7 (6), 1418-1424. 